\title{
The Load/Deflection Behavior of Pretensioned Cable/Pulley Transmission Mechanisms
}

by

\author{
Edward Ramsey Snow \\ B.S. Mechanical Engineering, Cornell University \\ (1986) \\ Submitted to the \\ Massachusetts Institute of Technology/ \\ Woods Hole Oceanographic Institution \\ Joint Program in Mechanical Engineering \\ in partial fulfillment of the \\ requirements for the degree of

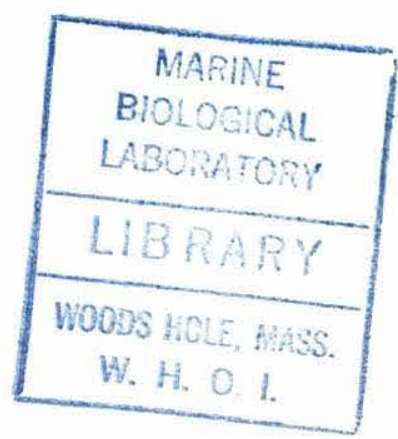 \\ MASTER OF SCIENCE IN MECHANICAL ENGINEERING \\ at the \\ MASSACHUSETTS INSTITUTE OF TECHNOLOGY \\ and the \\ WOODS HOLE OCEANOGRAPHIC INSTITUTION \\ December, 1993 \\ (C) Edward R. Snow, 1993 \\ The author hereby grants to MIT, WHOI, and the \\ U.S. Government permission to reproduce and \\ distribute copies of this thesis in whole or in part.
}

Signature of Author

Department of Mechanical Engineering, Massachusetts Institute of Technology/

Woods Hole Oceanographic Institution

Certified by

Dr. Dana R. Yoerger, Wogds Hole Oceanographic Institution, Thesis Supervisor

Accepted by

Dr. Arthur B. Baggeroer

Chairman, Joint Committee for Applied,

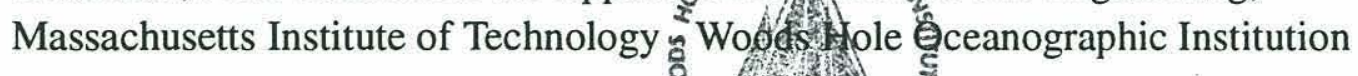




\title{
The Load/Deflection Behavior of Cable/Pulley \\ Transmission Mechanisms
}

\author{
by \\ Edward Ramsey Snow \\ Submitted to the \\ Massachusetts Institute of Technology/ \\ Woods Hole Program in Oceanography and Oceanographic Engineering \\ in partial fulfillment of the requirements of the Degree of \\ Master of Science
}

\begin{abstract}
Mechanical transmission mechanisms enable a designer to match the abilities (e.g. velocity, torque capacity) of an actuator to the needs of an application. Unfortunately the mechanical limitations of the transmission (e.g. stiffness, backlash, friction, etc.) often become the source of new problems. Therefore identifying the best transmission option for a particular application requires the designer to be familiar with the inherent characteristics of each type of transmission mechanism.

In this thesis we model load/deflection behavior of one particular transmission option; closed circuit cable/pulley transmissions. Cable drives are well suited to force and position control applications because of their unique combination of zero backlash motion, high stiffness and low friction. We begin the modelling process by determining the equilibrium elongation of a cable wrapped around a nonrotating pulley during loading and unloading. These results enable us to model the load/deflection behavior of the open circuit cable drive. Using the open circuit results we model the more useful closed circuit cable drive. We present experimental results which confirm the validity of both cable drive models and then extend these models to multistage drives. We end by discussing the use of these models in the design of force and position control mechanisms and comment on the limitations of these models.
\end{abstract}

Thesis Supervisor: Dana R. Yoerger 


\section{Table of Contents}

1 Introduction 11

1.1 Transmissions 11

$\begin{array}{lll}1.2 & \text { Problem Statement } & 12\end{array}$

1.3 Why Study Cable/Pulley Transmissions? 12

1.4 Introduction To Cable/Pulley Transmissions 13

$\begin{array}{ll}\text { 1.4.1 Open Circuit Cable Transmissions } & 13\end{array}$

1.4.2 Closed Circuit Cable/Pulley Transmissions 14

1.5 Summary of Results 14

1.6 Thesis Overview 15

2 The Mechanics of Wrapped Cable 17

2.1 Wrapped Cable on a Non-rotating Pulley 18

2.2 Modelling 20

2.2.1 The Slip Condition $\quad 20$

2.2.2 The Affected Angle of Wrap 24

2.2.3 Tension Profile in a Wrapped Cable 26

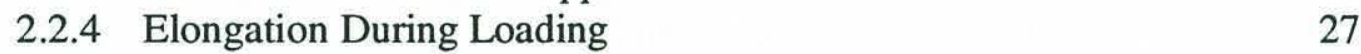

2.2.5 Elongation During Unloading 33

2.2.6 Energy Dissipated During Loading 39

2.2.7 Energy Stored During Loading $\quad 40$

2.3 Consistency Checks 41

2.3.1 Conservation of Energy 41

2.3.2 Efficiency Limit of Tension Element Drives 42

$\begin{array}{lll}2.4 & \text { Conclusions } & 49\end{array}$

3 Open Circuit Cable/Pulley Drives $\quad 51$

3.1 The Open Circuit Cable Drive $\quad 52$

3.2 Modelling 53

3.2.1 The Geometry-Friction Number 55

3.2.2 Deflection During Loading $\quad 57$

3.2.3 Deflection During Unloading 59

3.2.4 Comments on the Validity of Open Circuit Models 61

3.3 Experimental Confirmation 64

3.3.1 Approach 64

3.3.2 A Typical Trial 66

3.3.3 Correlation Coefficients: All Trials 68

3.3.4 Coefficient of Friction: All Trials 69

$\begin{array}{lll}3.4 & \text { Conclusions } & 71\end{array}$ 
4 Closed Circuit Cable/Pulley Drives

4.1 The Closed Circuit Cable Drive $\quad 74$

4.2 Modelling $\quad 75$

4.2.1 Equilibrium Equations During Loading 77

4.2.2 Equilibrium Equations During Unloading 78

4.2.3 Special Case I: Widely Separated Pulleys 80

4.2.4 Special Case II: Narrowly Separated Pulleys 83

4.2.5 General Case Solutions $\quad 88$

4.2.6 Approximate Solutions for the General Case 93

$\begin{array}{lll}4.3 & \text { Experimental Confirmation } & 97\end{array}$

4.3.1 Approach 97

4.3.2 A Typical Trial 99

4.3.3 Correlation Coefficients: All Trials 100

4.3.4 Coefficient of Friction: All Trials 101

$\begin{array}{lll}4.4 & \text { Conclusions } & 102\end{array}$

5 Multistage Drives $\quad 105$

5.1 Multistage Open Circuit Drives 106

5.2 Multistage Closed Circuit Drives 110

5.2.1 Two Antagonistic Multistage Open Circuit Drives in Parallel 111

5.2.2 Individual Closed Circuit Drives in Series 112

$\begin{array}{lll}5.3 \text { Conclusions } & 115\end{array}$

6 Designing Cable Drives 117

$\begin{array}{lll}6.1 & \text { Suitable Applications } & 118\end{array}$

6.2 Choosing the Appropriate Model for a Given Cable Drive 120

6.3 Performance Characteristics and Design Parameters 121

6.3.1 Performance Characteristics 122

6.3.2 Design Parameters 124

6.4 Limitations of the Models/ Future Work 126

A Experimental Apparatus and Procedures 127

$\begin{array}{lll}\text { A.1 Apparatus } & 127\end{array}$

$\begin{array}{ll}\text { A.2 Experimental Procedure } & 129\end{array}$

$\begin{array}{ll}\text { A.3 Torque Sensor Compliance } & 130\end{array}$ 
B Coefficients of Friction for Open and Closed Circuit Experiments 133

B.1 True Coefficient of Friction, Open Circuit Trials 133

B.2 Trials 36 through 85: Single Pulley Tests 134

B.3 Trials 86 through 110: Single Pulley Tests 135

B.4 Trials 111 through 135: Single Pulley Tests 136

B.5 Trials 136 through 172: Dual Pulley Tests 137

B.6 Trials 173 through 188: Dual Pulley Tests 138

B.7 Closed Circuit Experiments: Trials 1 through 30: Dual Pulley Tests 139

C Special Case II: Exact Tensions During Unloading 141

$\begin{array}{ll}\text { References } & 145\end{array}$ 


\section{List of Figures}

1 Introduction 11

1.1 Open Circuit Cable/Pulley Transmission 13

1.2 Closed Circuit Cable/Pulley Transmission 14

2 The Mechanics of Wrapped Cable 17

2.1 Linearly Elastic Cable Wrapped Around a Non-rotating Pulley 18

$\begin{array}{ll}2.2 \text { Schematic of Approach } & 19\end{array}$

2.3 Free Body Diagram of Wrapped Cable Segment 20

2.4 Wrapped Cable Under Load 24

2.5 Tensile Profile During Loading $\quad 27$

2.6 Schematic of Wrapped Cable During Loading 28

2.7 Elongation of Wrapped Cable on a Non-rotating Pulley 31

2.8 Stiffness of Wrapped Cable on a Non-rotating Pulley 32

2.9 Schematic of Wrapped Cable During Unloading 34

2.10 Tensile Profile During Unloading 37

2.11 Continuously Driven Pulley Under Load 43

2.12 Affected Angles of Wrap and Tensile Profiles 45

3 Open Circuit Cable/Pulley Drives 51

3.1 Single Stage Pretensioned Open Circuit Drive 52

3.2 Deflection of an Open Circuit Drive During Loading 57

3.3 Stiffness of an Open Circuit Drive During Loading 58

3.4 Deflection of an Open Circuit Cable Drive During Unloading, GF=0 60

3.5 Wrap Deflection and Dimensionless Moment vs. Time, Trial 145

3.6 Dimensionless moment vs. Normalized Deflection, Trial $145 \quad 67$

$3.7 \ell_{1}$ and $t_{u}$ vs. $\theta_{w r}$, Trial $145 \quad 68$

3.8 Correlation Coefficients, Trials 36 through 188

4 Closed Circuit Cable/Pulley Drives 73

4.1 Closed Circuit Cable Drive. 74

4.2 Closed Circuit Drive with Widely Separated Pulleys 80

4.3 Closed Circuit Drive with Narrowly Separated Pulleys 83

4.4 Fixed Load Stiffness vs. Initial Pretension 85

4.5 Equilibrium Tensions During Loading and Unloading when 86

4.6 Affected Angles of Wrap and Tension Bump Locations 87

4.7 Equilibrium Deflections During Loading and Unloading When 88

4.8 Dimensionless Tension vs. Dimensionless Moment, Various Values of 89 
4.9 Dimensionless Tension vs. Dimensionless Moment, Various Values of 90 4.10 Normalized Affected Angles of Wrap vs. Dimensionless Moment 91

4.11 Dimensionless Moment vs.Normalized Deflection 92

4.12 Dimensionless Moment vs.Normalized Deflection. 93

4.13 Accuracy Approximate Model I without Wrapped Cable Term 94

4.14 Accuracy of Approximate Solution I with Wrapped Cable Term 95

4.15 Accuracy of Approximate Solution II 96

4.16 Dimensionless moment vs. Normalized Deflection, Trial 19

$4.17 k_{1}$ and $k_{u}$ vs. $\hat{\theta}_{\mathrm{wr}}$, Trial $19 \quad 100$

4.18 Correlation Coefficients, Trials 1 through $30 \quad 101$

5 Multistage Drives $\quad 105$

5.1 Multistage Open Circuit Cable Drive 106

5.2 Multistage Closed Circuit Drive Made from Two Multistage Open Circuit 111 Drives

5.3 Multistage Closed Circuit Drive Made from Cascaded Single Stage Closed 112 Circuit Drives

6 Designing Cable Drives 117

6.1 Crossed and Uncrossed Methods of Rigging Cable Drives 125

A Experimental Apparatus and Procedures 127

$\begin{array}{ll}\text { A.1 Experimental Apparatus } & 127\end{array}$

$\begin{array}{ll}\text { A.2 Detail of Experimental Apparatus } & 128\end{array}$

B Coefficients of Friction for Open and Closed Circuit Experiments 133

B.1 Loading (o) and Unloading (x) Coefficients of Friction, Trials 36-85 134

B.2 Loading (o) and Unloading (x) Coefficients of Friction, Trials 86-110 135

B.3 Loading (o) and Unloading (x) Coefficients of Friction, Trials 111-135 136

B.4 Loading (o) and Unloading (x) Coefficients of Friction, Trials 136-172 137

B.5 Loading (o) and Unloading (x) Coefficients of Friction, Trials 173-188 138

B.6 Loading (o) and Unloading (x) Coefficients of Friction, Trials 1-30 139

C Special Case II: Exact Tensions During Unloading 141 


\section{Chapter 1 Introduction}

\subsection{Transmissions}

Ideally transmissions would be unnecessary; actuators would be compact, efficient and powerful enough to be attached directly to the objects they control (an option known as direct drive). Unfortunately the limitations of existing actuators make this option infeasible for a wide range of applications. To make up for this we use transmissions to modify the apparent characteristics of an actuator. By combining an actuator with a properly designed transmission we create a new system whose abilities more closely match the characteristics of our ideal actuator. These characteristics can be divided into three categories.

\section{Output:}

Examples: Torque, velocity, shaft friction, etc.

Many actuators (electric motors, internal combustion engines) deliver power most efficiently at high velocity and low torque while many applications (robotics, earthmoving) require the application of high torques at low velocity. Using a transmission to boost the torque and reduce the velocity of the actuator we create an actuator/transmission assembly which resembles an actuator having the desired torque/velocity characteristics.

\section{Packaging:}

Examples: Physical volume, weight

An actuator, even if it has the desired output characteristics, may still be too heavy or cumbersome to be connected directly to a given load. Using a transmission allows the actuator to be separated from the load and placed where there is sufficient room or weight bearing capability, reducing the packaging demands at the load.

\section{Cost:}

We often find it cheaper to use a transmission to adapt a non-ideal actuator to a specific application than to procure an inherently capable actuator (assuming that a capable actuator even exists). In addition, it is typically cheaper to use one or more transmissions to deliver power from a single actuator to multiple loads than it is to assign a separate actuator to each load (assuming that the motions of the 


\section{Chapter 1: Introduction}

different loads are not independent).

Transmissions are not perfect, however. Each type of transmission (e.g. gears, belts, cables, chains, etc.) has its own particular physical limitations (e.g. backlash, friction, load capacity, etc.) which impact the new system's performance. As designers of transmissions we must select a transmission concept whose general characteristics suit our application and then tailor the specific characteristics of an implementable version to the task requirements.

\subsection{Problem Statement}

We seek in this report to identify and model the physical characteristics of closed circuit cable/pulley transmissions to assist the transmission designer in the evaluation, design, and optimization of cable/ pulley transmissions.

\subsection{Why Studv Cable/Pullev Transmissions?}

Cable/pulley transmissions combine zero backlash motion and high stiffness with uniquely low stiction/friction levels, a very desirable combination in force/torque control applications. Backlash severely impacts closed loop bandwidth (Schempf [11]) whereas stiction can limit the force resolution of a system by inducing limit cycling (Townsend and Salisbury [15]). High stiffness, of course, helps increase system bandwidth. As a consequence of these features we find cable drives used most frequently in two applications requiring high performance force/torque control; robotics (Schempf [11], Townsend [15], Salisbury[9], Dipietro [3]) and telerobotics (Goertz[5], Vertut[16], Bejczy [1], McAffee et.al. [7])

To remove backlash from a transmission we typically preload the mechanism by adjusting the geometry of the mechanism until the drive elements have a slight interference fit. This removes the backlash but tends to increase frictional forces due to increased internal loads. Comparing five such zero backlash transmission mechanisms (a cable drive, a harmonic drive, a Kamo ball reducer, and cycloidal-type reducers manufactured by Dojen, Sumitomo and Redex) Schempf [11] found that the cable drive incurred the lowest friction penalty for removing backlash.

In robotic and telerobotic applications small collisions and impacts which occur during assembly and grasping tasks result in step changes in transmission loads, typically from near zero working load to some low amplitude peak load. To accurately transmit or respond to these changes the transmission must have a high force/torque bandwidth at low loads. Schempf [11] found that all of the above transmissions except the cable drive exhibited stiffening spring behavior, showing minimum stiffness (and therefore minimum band- 
width) near zero working load. In contrast (as we will show) the cable drive exhibits softening spring behavior, having its maximum stiffness (and therefore maximum bandwidth) at zero working load. Therefore, qualitatively speaking, the cable drive is better suited to these applications.

\subsection{Introduction To Cable/Pullev Transmissions}

There are two different types of cable/pulley transmissions; open circuit and closed circuit drives. To familiarize the reader we briefly describe each below.

\subsubsection{Open Circuit Cable Transmissions}

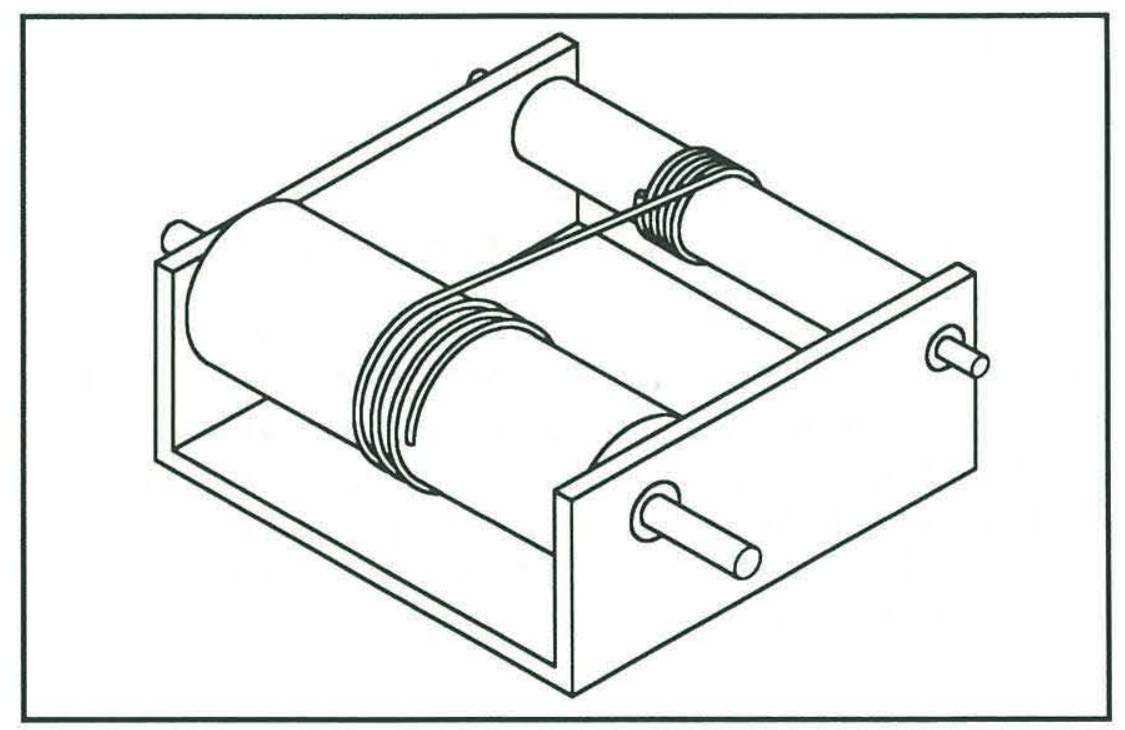

Figure 1.1. Open Circuit Cable/Pulley Transmission

Figure 1.1. shows a simple cable drive consisting of two pulleys linked by a single piece of cable. This is an open circuit cable drive, defined as a drive whose cable(s) are slack unless opposing torques are applied to the input and output pulleys. For the drive to function in both directions we must preload or pretension the drive such that the cable never goes slack, for example, by hanging opposing counterweights from each pulley or using opposing actuators at each pulley. Our interest in the open circuit drive stems less from its limited practical applications than from the considerable insight its closed form models give us into the behavior of the more useful (but more complex) closed circuit drive. 


\section{Chapter 1: Introduction}

\subsubsection{Closed Circuit Cable/Pulley Transmissions}

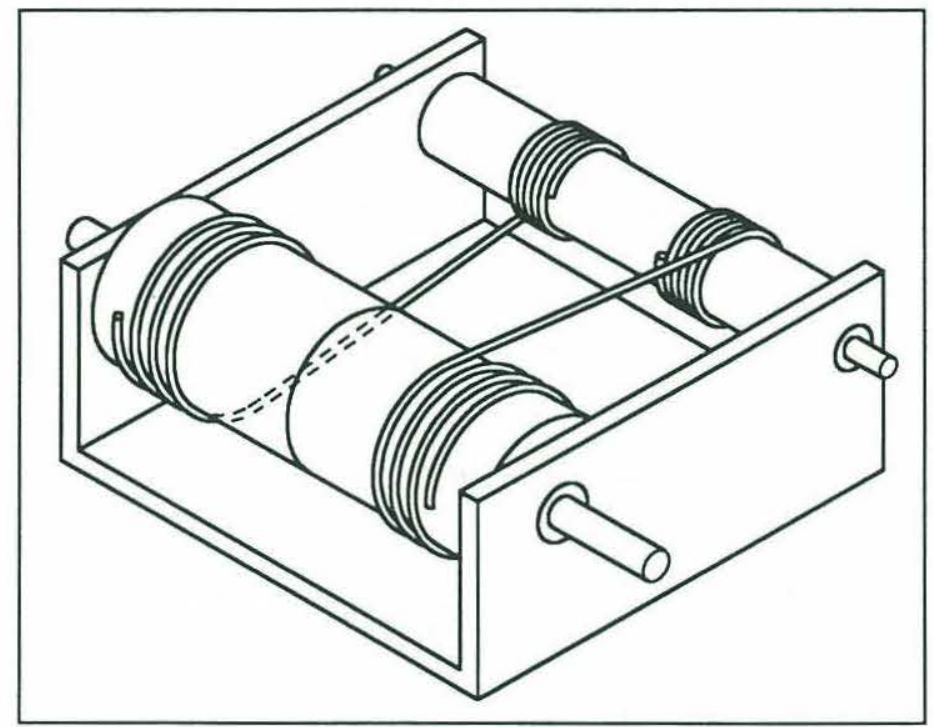

Figure 1.2. Closed Circuit Cable/Pulley Transmission

In this drive two pieces of cable antagonistically link the input and output pulleys. We define a closed circuit cable transmission as a drive whose cables and pulleys form a closed force path that is under tension even when no torques act upon the input and output pulleys. By definition a closed circuit drive must be pretensioned. For the drive shown, we tension the two cables by turning the two halves of the large pulley with respect to each other and then clamping the pulley halves together to lock the circuit in a state of tension. Once pretensioned, this transmission allows the input to actively drive the output in both directions with no backlash.

\subsection{Summary of Results}

\section{Existing Cable Drive Model}

The cable drive models presented in this report are presented as an improvement over the first order model commonly in use today (this model neglects the elongation of the wrapped cable in the drive). The predicted drive characteristics associated with this model are a constant, linear stiffness, no hysteresis deflection and a minimum required pretension (i.e. the pretension required to prevent either cable from going slack during operation) of $\mathrm{T}_{0}=\frac{\mathrm{M}_{\max }}{2 \mathrm{R}}$ where $\mathrm{M}_{\max }$ is the maximum applied load and $\mathrm{R}$ is the radius of the output pulley. 
Thesis Overview

\section{New Cable Drive Model}

The new models predict substantially different behavior which we briefly summarize here. These characteristics have been verified directly or indirectly by the deflection experiments presented in Chapters 3 and 4.

Softening spring behavior: Cable drives have very high stiffness at low loads and markedly reduced stiffness levels at higher loads which drop off rapidly as the applied load increases. In addition, the stiffness also depends on the load history of the drive.

The Geometry-Friction number: This dimensionless parameter determines the nature of a cable drive's response to load (i.e. how nonlinear the behavior is). We encounter this number in the analysis of every drive investigated in this report and it is the key to modelling a given drive correctly.most important piece of information needed to

Hysteresis deflection: Hysteresis deflection is inherent to all cable drives. Surprisingly it is accurately predictable.

Dependence on pretension: The stiffness of an existing cable drive can be increased simply by increasing the initial pretension. This also reduces the hysteresis deflection remaining after an applied load has been removed.

Minimum pretension: Depending on the drive's configuration the minimum pretension required to prevent either cable from going slack during operation can be as low as $\mathrm{T}_{0}=\frac{\mathrm{M}_{\mathrm{max}}}{4 \mathrm{R}}$, one half that predicted by the existing model.

Maximum cable tensions: The maximum cable tensions under load can be as much as $30 \%$ higher than those obtained from existing cable drive models.

\subsection{Thesis Overview}

Chapter 1: Introduction: We describe the context of this work and discuss other relevant work. We present the problem statement and outline the remainder of the report.

Chapter 2: Mechanics of Wrapped Cable: We model the behavior of cable wrapped around a nonrotating pulley and show these results to be consistent previous results.

Chapter 3: Open Circuit Cable/Pulley Drives: We use the wrapped cable results from Chapter 2 to model and explain the load/deflection behavior of single stage open circuit drives. These are closed form models, allowing us to 
investigate the dependence of the stiffness on particular design parameters. We introduce the Geometry-Friction number GF, a dimensionless parameter which determines the character of a drive's response to load. We present experimental results confirming these models.

Chapter 4: Closed Circuit Cable/Pulley Drives: We model single stage closed circuit drives as two antagonistically combined open circuit drives and find that the Geometry-Friction number also determines the character of a closed circuit drive's response to load. In general we must solve numerically, but we investigate two limiting cases for which there are analytical solutions and show that one applies to the bulk of practical drives. We confirm this approximate model experimentally.

Chapter 5: Multistage Drives: We show that a multistage open circuit drive behaves exactly like an equivalent single stage open circuit drive whose GF number is a function of the properties of the individual stages. We identify two types of multistage closed circuit drives; one can be modeled as an equivalent single stage closed circuit drive, the other must be modeled as $n$ single stage closed circuit drives in series.

Chapter 6: Designing Cable Drives: We start by identifying cable drives as excellent transmissions for high performance position and force control applications. We reiterate the importance of the Geometry-Friction number and summarize its usefulness in the determining the appropriate model to use for a particular drive. After discussing the impact of the design parameters on the drive's performance characteristics we end by summarizing the limitations of these models. 


\section{Chapter 2 The Mechanics of Wrapped Cable}

To model a closed circuit cable drive we must be able to model the cable wrapped on its pulleys. In this chapter we model the load/deflection behavior of a cable wrapped around a non-rotating pulley. We show that changing the load applied to the cable's free end affects only a portion of the wrapped cable and that the cable tension in this region varies exponentially with position. By integrating the strain associated with this profile we obtain the elongation resulting from the change in applied load. We further demonstrate that the cable does not return to its original length when we return the applied load to its initial value.

To check these results we verify that energy is conserved during elongation (i.e. that the work done by the applied load equals the work required to stretch the cable). As an additional check we use the models to derive the efficiency limit for cable/flat belt drives, showing that the result agrees with the findings of others. 


\subsection{Wrapped Cable on a Non-rotating Pullev}

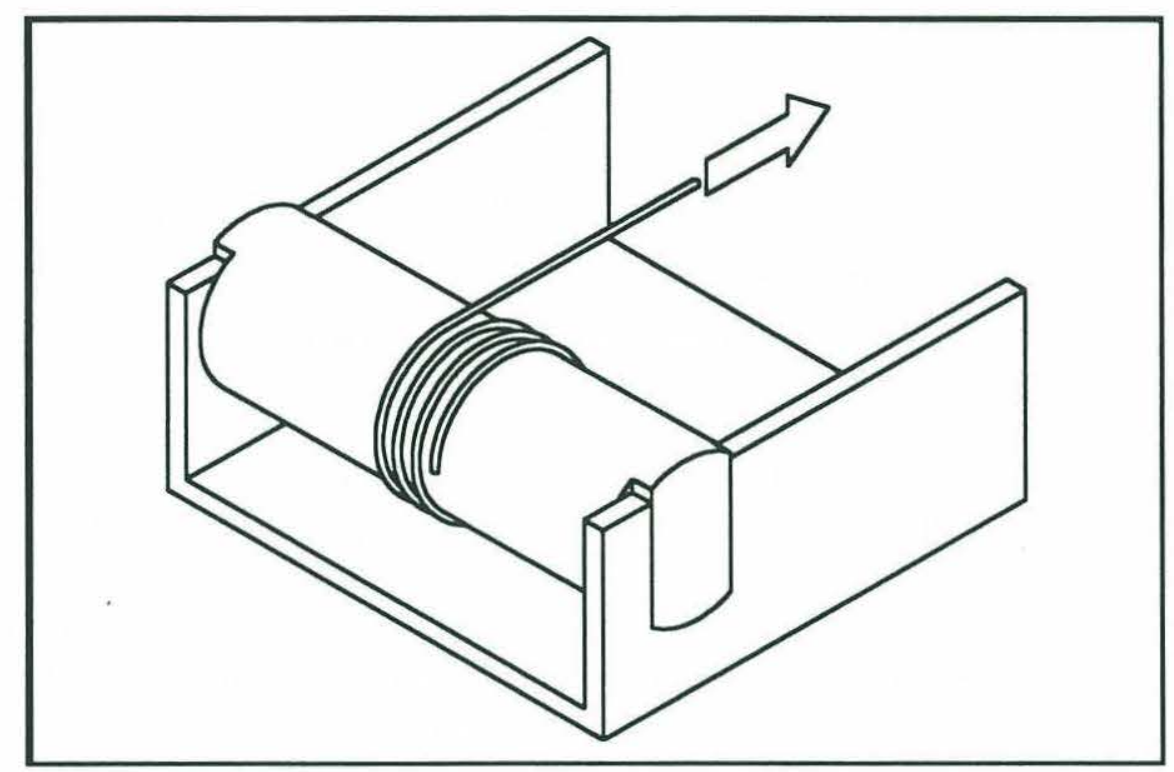

Figure 2.1. Linearly Elastic Cable Wrapped Around a Non-rotating Pulley

To develop a model for the load/deflection behavior of wrapped cable we analyze the system shown in Figure 2.1. Attaching one end of the cable to the pulley we wrap the rest such that the cable is uniformly pretensioned, i.e. such that the tension is everywhere equal to some value $\mathrm{T}_{0}$. At some later time we change the load applied to the cable's free end from $T_{0}$ to $T_{\text {app }}$, allow the system to reach equilibrium and then return the applied load to its original value $T_{0}$. We wish to determine the changes in the length of the wrapped cable resulting from these changes in applied load.

\section{Approach:}

The change in cable length (i.e. the elongation) is the integral of the change in cable strain. Determining the strain requires that we find the equilibrium tensile profile, which in turn depends on the amount of the wrapped cable affected by a change in applied load. To affect its neighbor a segment of wrapped cable must slip, i.e. the net load applied to it must exceed the frictional force acting upon it. Therefore we must start by determining the load conditions under which an arbitrary segment of wrapped cable will slip before we can find the affected angle of wrap. We summarize these dependencies in figure Figure 2.2. To find the elongation we simply start at the bottom of the list and work back towards the top. 


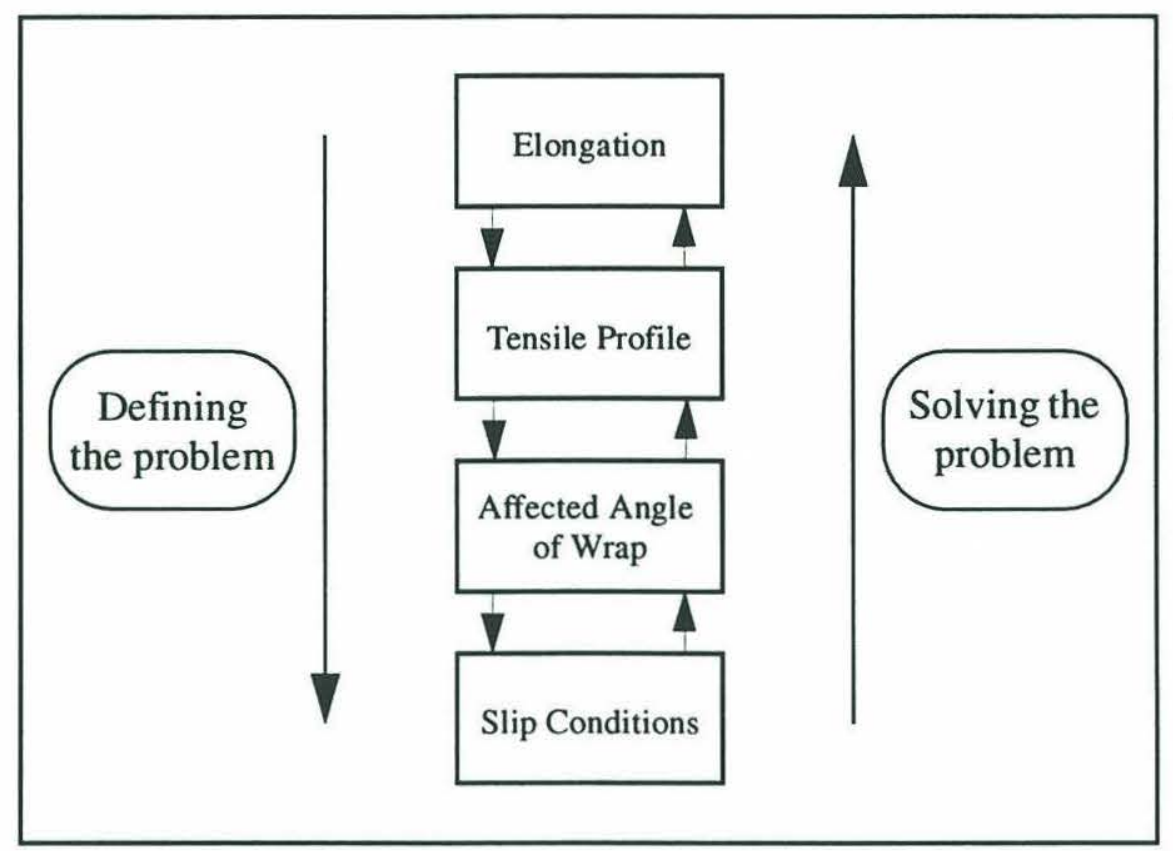

Figure 2.2. Schematic of Approach

\section{Chapter Overview:}

We attack each of these steps in a different subsection of this chapter. In the last two sections we check our results for consistency with the results of others.

\subsection{Modelling}

2.2.1 Slip Condition

2.2.2 Affected Angle of Wrap

2.2.3 Tensile Profile

2.2.4 Elongation During Loading

2.2.5 Elongation During Unloading

2.2.6 Energy Dissipated During Loading

2.2.7 Energy Stored During Loading

2.3 Consistency Checks

2.3.1 Conservation of Energy

2.3.2 Efficiency Limit of Tension Element Drives 


\subsection{Modelling}

\subsubsection{The Slip Condition}

Friction between the cable and the pulley enables the cable in Figure 2.3. to remain in equilibrium even if tensions $T_{1}$ and $T_{2}$ are not equal. We wish to determine how unequal these loads must be for the cable to slip freely with respect to the pulley. In the next section we use this result, first derived by Euler [4], to determine the portion of wrapped cable in Figure 2.1. affected by a change in applied load.

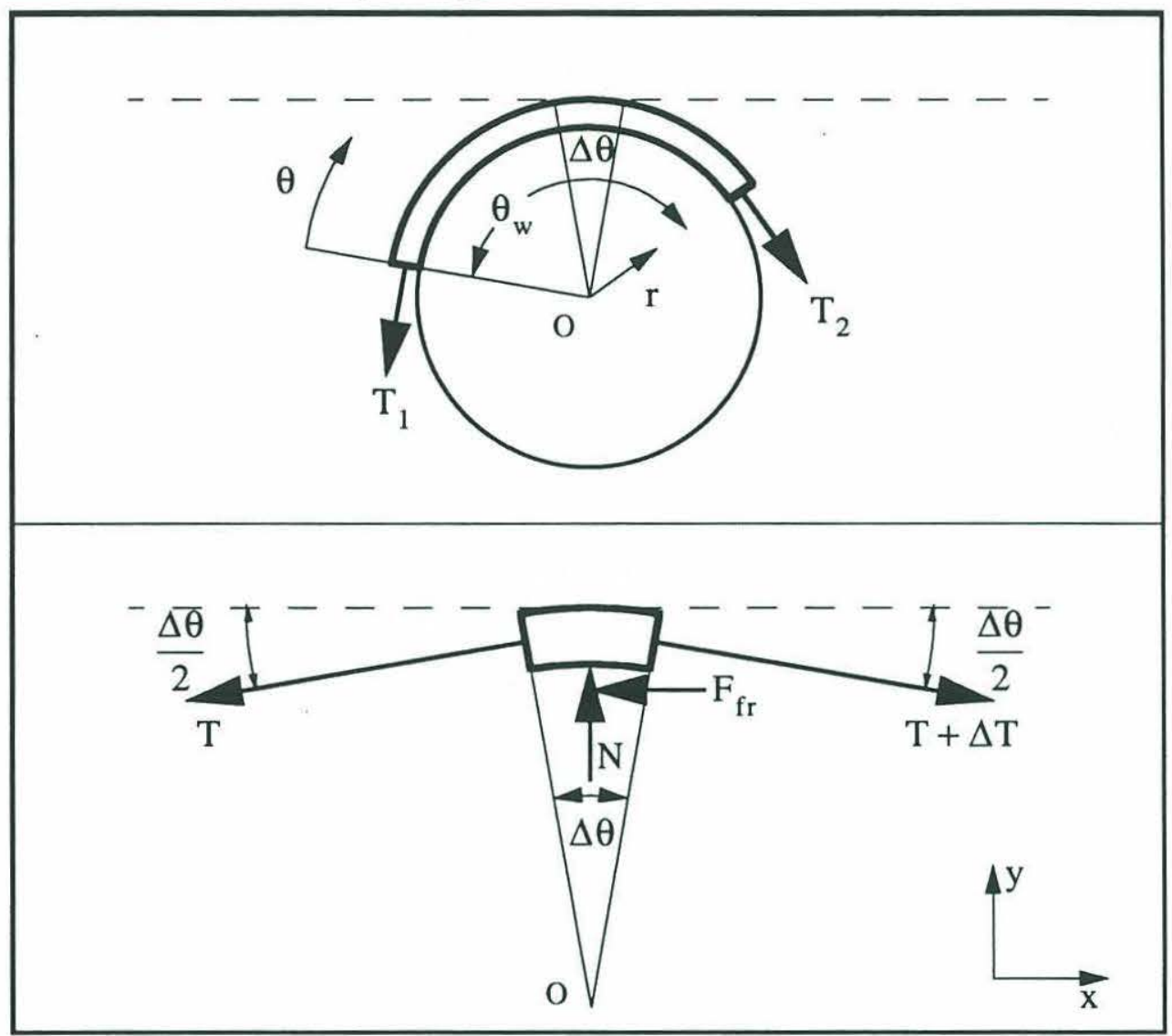

Figure 2.3. Free Body Diagram of Wrapped Cable Segment

We begin by looking at the equilibrium conditions for the differential element of cable $\Delta \theta$ shown in the lower half of Figure 2.3. Summing the forces and moments acting on the element we find that the cable will be in equilibrium (i.e. will not slip) if

$$
\Sigma \mathrm{F}_{\mathrm{x}}=0=(\mathrm{T}+\Delta \mathrm{T}) \cos \left(\frac{\Delta \theta}{2}\right)-\mathrm{T} \cos \left(\frac{\Delta \theta}{2}\right)-\mathrm{F}_{\mathrm{fr}}
$$




$$
\begin{aligned}
& \Sigma \mathrm{F}_{\mathrm{y}}=0=\mathrm{N}-(\mathrm{T}+\Delta \mathrm{T}) \sin \frac{\Delta \theta}{2}-\mathrm{T} \sin \frac{\Delta \theta}{2} \\
& \Sigma \mathrm{M}_{\mathrm{O}}=0=\mathrm{F}_{\mathrm{fr}} \mathrm{R}+\mathrm{TR}-(\mathrm{T}+\Delta \mathrm{T}) \mathrm{R}
\end{aligned}
$$

where the coordinates $\mathrm{x}$ and $\mathrm{y}$ are aligned with the tangential and normal directions for the element. Note that we have assumed that $d$ « $R$ (where $d$ is the cable diameter) and that the stress in the cable is uniaxial and does not vary significantly over a cross section of the cable (which should be valid if $d$ \& $R$ is valid).

As we shrink the size of the element from $\Delta \theta$ to $\mathrm{d} \theta$, the change in tension $\Delta \mathrm{T}$ across the element becomes $d T$. Since $d \theta$ is very small $\cos \left(\frac{d \theta}{2}\right) \approx 1$ and $\sin \left(\frac{d \theta}{2}\right) \approx \frac{d \theta}{2}$. Making these substitutions the equilibrium constraints become

$$
\begin{aligned}
& \Sigma \mathrm{F}_{\mathrm{x}}=\mathrm{dT}-\mathrm{F}_{\mathrm{fr}}=0 \\
& \Sigma \mathrm{F}_{\mathrm{y}}=\mathrm{N}-(2 \mathrm{~T}-\mathrm{dT}) \frac{\mathrm{d} \theta}{2}=0 \\
& \Sigma \mathrm{M}_{\mathrm{O}}=\left[\mathrm{F}_{\mathrm{fr}}-\mathrm{dT}\right] \mathrm{R}=0
\end{aligned}
$$

Recognizing the equivalence of the $\Sigma \mathrm{F}_{\mathrm{x}}$ and $\Sigma \mathrm{M}_{\mathrm{O}}$ equations and dropping the second order term $-\frac{\mathrm{dTd} \theta}{2}$ from equation (2-5) the equilibrium equations for the differential element of cable become

$$
\begin{aligned}
& F_{f r}=d T \\
& N=T d \theta
\end{aligned}
$$

Assuming the Coulomb model of friction applies we can say that

$$
\left|\mathrm{F}_{\mathrm{fr}}\right| \leq \mu \mathrm{N}=\mu \mathrm{Td} \theta
$$

where $\mu$ is the coefficient of friction between the cable and pulley. Using equation (2-7) to eliminate $F_{f r}$ from (2-9) we get

$$
|\mathrm{dT}| \leq \mu \mathrm{Td} \theta
$$


$\mu \mathrm{Td} \theta$ is the maximum supportable tension difference (i.e. net force) that the element can resist without slipping. In general a differential element of cable will not slip as long as

$$
\frac{|\mathrm{dT}|}{\mathrm{d} \theta}=\left|\frac{\mathrm{dT}}{\mathrm{d} \theta}\right| \leq \mu \mathrm{T}
$$

We call equation (2-11) the differential slip condition. It states that a differential element of cable will not slip until the magnitude of the slope of its tension $\frac{d T}{d \theta}$ exceeds the product of the local tension $\mathrm{T}$ and the coefficient of friction $\mu$. Conversely, an element of cable will continue to slip until the magnitude of the tensile slope $\frac{\mathrm{dT}}{\mathrm{d} \theta}$ equals $\mu \mathrm{T}$.

To find the load capacity of the entire arc of cable we sum the individual load capacities of every element in the cable by integrating the differential slip condition. Rearranging terms and recognizing that the tension $\mathrm{T}$ is always positive we can write

$$
\frac{|\mathrm{dT}|}{\mathrm{T}}=\left|\frac{\mathrm{dT}}{\mathrm{T}}\right| \leq \mu \mathrm{d} \theta
$$

Integrating from one end of the angle of wrap to the other we get

$$
\left|\int_{\mathrm{T}_{1}}^{\mathrm{T}_{2}} \frac{\mathrm{dT}}{\mathrm{T}}\right| \leq \int_{0}^{\theta_{\mathrm{w}}} \mu \mathrm{d} \theta
$$

This leads to two different solutions, the result depending on the sign of dT.

If $T_{2}>T_{1} d T$ is positive and we get

$$
\begin{gathered}
\log \left(\frac{\mathrm{T}_{2}}{\mathrm{~T}_{1}}\right) \leq \mu \theta_{\mathrm{w}} \\
\frac{\mathrm{T}_{2}}{\mathrm{~T}_{1}} \leq \mathrm{e}^{\mu \theta_{\mathrm{w}}}
\end{gathered}
$$

If $T_{2}<T_{1} d T$ is negative and we get 


$$
\frac{\mathrm{T}_{2}}{\mathrm{~T}_{1}} \leq \mathrm{e}^{-\mu \theta_{\mathrm{w}}}
$$

Equations (2-15) and (2-16) are well known results, first derived by Euler [4] in 1765. To represent both cases with a single equation we introduce the signed coefficient of friction $\mu^{*}$, defined as

$$
\mu^{*} \equiv \mu \operatorname{sgn}\left(\frac{\mathrm{T}_{2}}{\mathrm{~T}_{1}}-1\right)
$$

where $T_{2}$ is the tension acting in the positive $\theta$ direction. Physically $\mu^{*}$ reflects the fact that the frictional force $\mathrm{F}_{\mathrm{fr}}$ always opposes the net force acting on the cable. Thus we find that a wrapped cable will not slip as long as

$$
\frac{\mathrm{T}_{2}}{\mathrm{~T}_{1}} \leq \mathrm{e}^{\mu^{*} \theta_{\mathrm{w}}}
$$

Rearranging terms we can also write this as

$$
\theta_{\mathrm{w}} \geq \frac{1}{\mu^{*}} \log \left(\frac{\mathrm{T}_{2}}{\mathrm{~T}_{1}}\right)
$$

We call this constraint the integral slip condition. We define the slip angle $\phi$ as the minimum angle of wrap required to support the applied loads, i.e.

$$
\phi \equiv \frac{1}{\mu^{*}} \log \left(\frac{\mathrm{T}_{2}}{\mathrm{~T}_{1}}\right)
$$

$\phi$ corresponds to the case when every element supports the maximum tension difference that it can withstand, (i.e.when $\frac{\mathrm{dT}}{\mathrm{d} \theta}=\mu^{*} \mathrm{~T}(\theta)$ for every element). Thus an arc of wrapped cable will not slip as long as

$$
\theta_{\mathrm{w}} \geq \phi
$$




\subsubsection{The Affected Angle of Wrap}

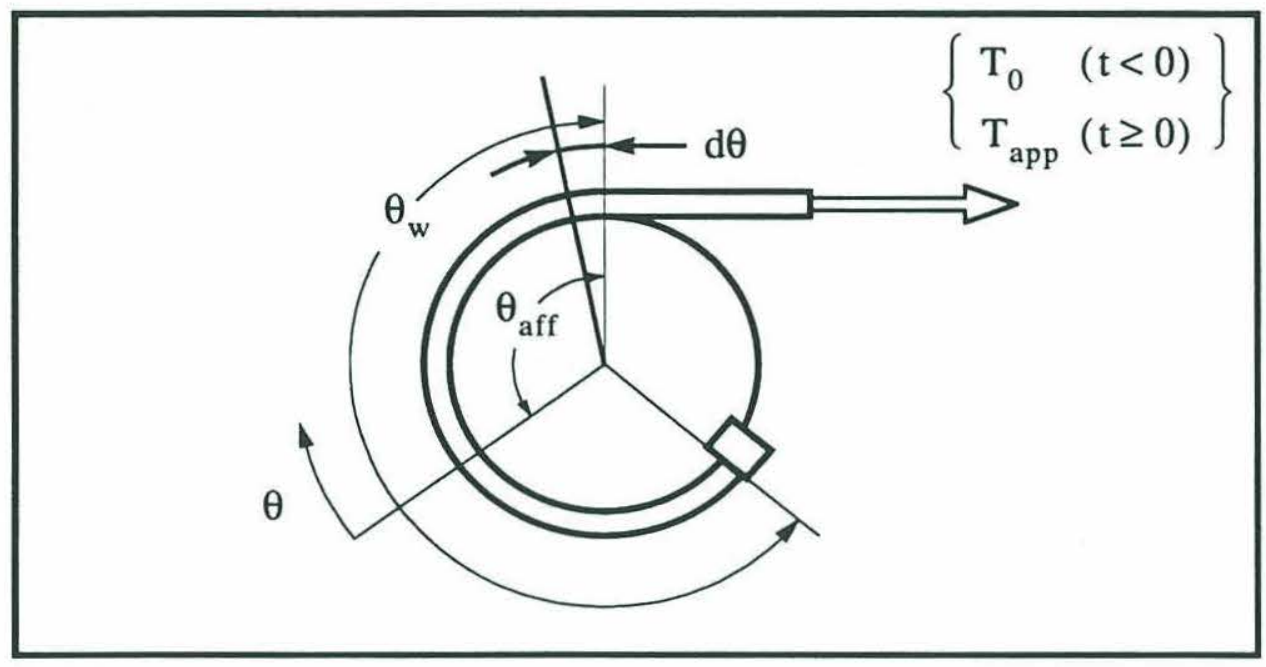

Figure 2.4. Wrapped Cable Under Load

Returning to the system shown in Figure 2.1. we wish to find the affected angle of wrap $\theta_{\text {aff }}$, defined as the region of cable whose tension changes from its initial value $T_{0}$ when we change the load applied to the cable's free end from $T_{0}$ to $T_{\text {app }}$.

We begin by considering a differential element of cable $\mathrm{d} \theta$ located at the cable's point of departure. The differential slip condition (2-11) states that the element will not slip if

$$
\left|\frac{\mathrm{dT}}{\mathrm{d} \theta}\right| \leq \mu \mathrm{T}_{0}
$$

At the instant we change the load the tensions acting on either side of the element $\mathrm{d} \theta$ differ by the finite amount $T_{a p p}-T_{0}$ which means that the slope $\frac{d T}{d \theta}= \pm \infty$. Thus the element must slip and (since any change in applied load, however small, results in an infinite slope) and it starts to do so the as soon as the applied load begins to change. During slip the change in tension across the element equals the frictional force acting on the element or

$$
\mathrm{dT}=\mathrm{F}_{\mathrm{fr}}=\mu_{\mathrm{d}}^{*} \mathrm{~T}_{\mathrm{app}} \mathrm{d} \theta
$$

where the $\mu_{\mathrm{d}}^{*}$ is the signed version of the dynamic coefficient of friction. Consequently the tensions acting on the adjacent element now differ by the amount $\left(\mathrm{T}_{\mathrm{app}}-\mathrm{F}_{\mathrm{fr}}\right)-\mathrm{T}_{0}$ and, by the same argument presented above, this element must also slip. The affected region 
continues to grow in this way until the sum of the frictional forces acting on the affected elements exactly equals the change in applied load, i.e. until $\theta_{\text {aff }}$ reaches equilibrium. In the previous section we showed that to be in equilibrium an arc of cable must satisfy the aggregate slip condition (2-19). Applying that result we see that $\theta_{\text {aff }}$ stops growing when

$$
\theta_{\text {aff }}=\phi=\frac{1}{\mu^{*}} \log \frac{T_{\text {app }}}{\mathrm{T}_{0}}
$$

where

$$
\mu^{*}{ }_{\mathrm{d}}=\mu_{\mathrm{d}} \operatorname{sgn}\left(\frac{\mathrm{T}_{\mathrm{app}}}{\mathrm{T}_{0}}-1\right)
$$

(Cotterill [2], in his 1892 study of flat belt power transmissions, appears to have been the first to recognize that $\theta_{\text {aff }}=\phi$ ). Obviously equation (2-24) only applies if $\theta_{w}>\phi$. If $\theta_{w}<\phi$ the affected angle of wrap is clearly

$$
\theta_{\mathrm{aff}}=\theta_{\mathrm{w}}
$$

For all the systems analyzed in this report we assume that $\theta_{w}>\phi$, i.e. that equation (2-24) applies. There are several important implications of equations (2-24) and (2-26);

1. Wrapped cable exhibits no stick/slip behavior during loading

2. Wrapped cable's equilibrium behavior depends on the dynamic coefficient of friction $\mu_{\mathrm{d}}$

3. If $\theta_{\mathrm{w}}>\phi$ changes in applied load do not affect the tension at the cable termination point (i.e. the tension at the termination is always $\mathrm{T}_{0}$ ).

4. If $\theta_{\mathrm{w}}>\phi$ the response of wrapped cable to applied loads is independent of the angle of wrap $\theta_{\mathrm{w}}$.

It may seem odd that the results depend on $\mu_{\mathrm{d}}^{*}$ instead of the static coefficient of friction $\mu_{s}^{*}$. To understand this we consider the last element to be affected before $\theta_{\text {aff }}$ reaches equilibrium (i.e. the element at $\theta=0$ ). Once its tension changes the element responds by increasing or decreasing in length. As this happens every other element in the affected region must also slip by this amount, meaning that every element in the entire affected region is slipping just prior to reaching equilibrium. During slip the dynamic coefficient of 
friction $\mu_{\mathrm{d}}{ }^{*}$ determines the frictional force acting on the elements. These forces act on the elements until the region reaches its final elongation, at which point the tensile profile associated with $\mu_{\mathrm{d}}{ }^{*}$ is locked in place by the higher static coefficient of friction $\mu_{\mathrm{s}}^{*}$. By applying the same arguments one can show that $\mu_{\mathrm{d}}{ }^{*}$ also determines the response to any further changes in the applied load. Thus the behavior of a wrapped cable at static equilibrium is determined by the dynamic coefficient of friction $\mu_{\mathrm{d}}{ }^{*}$. (These results tell us that we should use $\mu_{\mathrm{d}}{ }^{*}$ in the integral slip condition (2-19)). Unless otherwise noted the use of $\mu$ in this report refers to the dynamic coefficient of friction $\mu_{\mathrm{d}}$.

\subsubsection{Tension Profile in a Wrapped Cable}

We shall now determine the equilibrium tensile profile $T(\theta)$ in the affected region after changing the applied load from $\mathrm{T}_{\mathrm{o}}$ to $\mathrm{T}_{\mathrm{app}}$.

While determining the affected angle of wrap we showed that the equilibrium tension difference across a differential element of cable in the affected region is

$$
\mathrm{dT}=\mu^{*} \mathrm{Td} \theta
$$

where $\mu^{*}=\mu_{d}^{*}$ as given by equation (2-25). To solve for the tension $\mathrm{T}(\theta)$ at position $\theta$ we rearrange terms and integrate from $\theta=0$ to $\theta=\theta$ to get

$$
\begin{gathered}
\int_{\mathrm{T}_{0}}^{\mathrm{T}(\theta)} \frac{\mathrm{dT}}{\mathrm{T}}=\int_{0}^{\theta} \mu^{*} \mathrm{~d} \theta \\
\log \left(\frac{\mathrm{T}(\theta)}{\mathrm{T}_{0}}\right)=\mu^{*} \theta \\
\mathrm{T}(\theta)=\mathrm{T}_{0} \mathrm{e}^{\mu^{*} \theta}
\end{gathered}
$$

where $0 \leq \theta \leq \theta_{\text {aff }}=\phi$ and we measure $\theta$ from the interior edge of the affected angle of wrap as shown in Figure 2.4.

Recalling that $\mu^{*}=\mu \operatorname{sgn}\left(\frac{\mathrm{T}_{\text {app }}}{\mathrm{T}_{0}}-1\right)$ we see that the tension profile varies exponentially 
from $\theta=0$ to $\theta=\phi$, decaying when $\mathrm{T}_{\text {app }}<\mathrm{T}_{0}$ and increasing when $\mathrm{T}_{\text {app }}>\mathrm{T}_{0}$ (we show the latter case in Figure 2.5.) Euler[4] was the first to show that equation (2-30) applies to the cable in Figure 2.3. just prior to slip. We believe Cotterill [2] was the first to recognize that it applies to a belt (or cable) at static equilibrium on a stationary pulley

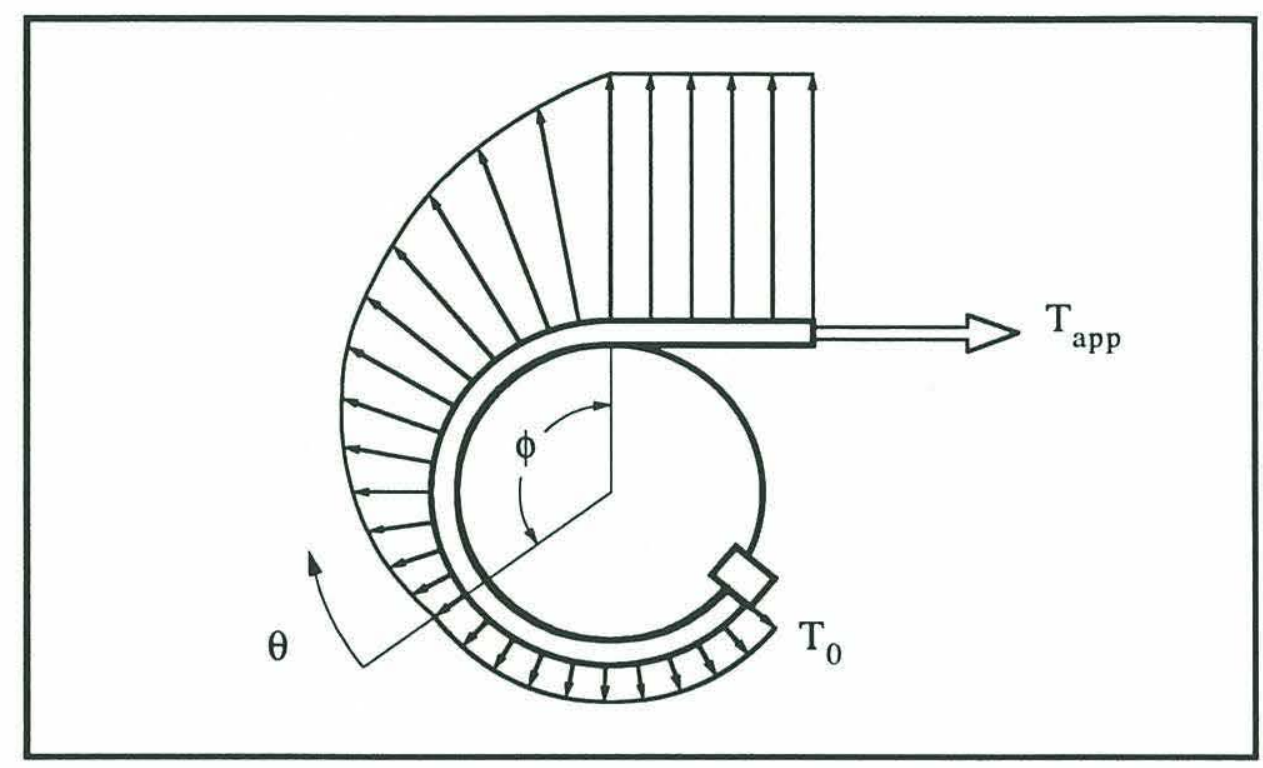

Figure 2.5. Tensile Profile During Loading

\subsubsection{Elongation During Loading}

Referring to Figure 2.6., we wish to determine how changing the applied load from $\mathrm{T}_{0}$ to $\mathrm{T}_{\text {app }}$ affects the elongation $\delta$ of the wrapped portion of the cable. To find $\delta$ we sum the change in elongation of the all the differential elements in the cable. For an element defined by an angle $\Delta \theta$ this change $\Delta \delta$ is

$$
\Delta \delta=\left(\varepsilon_{\mathrm{eq}}-\varepsilon_{0}\right) \mathrm{R} \Delta \theta
$$

where $\varepsilon_{\mathrm{eq}}$ is the total strain in the element at equilibrium, $\varepsilon_{0}$ is the initial strain associated with the pretension $T_{0}$ and $R \Delta \theta$ is the unstressed length of the element. As we shrink the size of the element from $\Delta \theta$ to $d \theta$, the change in the element's length $\Delta \delta$ across the element approaches $\mathrm{d} \delta$ and (2-31) becomes

$$
\mathrm{d} \delta=\left(\varepsilon_{\mathrm{eq}}-\varepsilon_{0}\right) \operatorname{Rd} \theta
$$




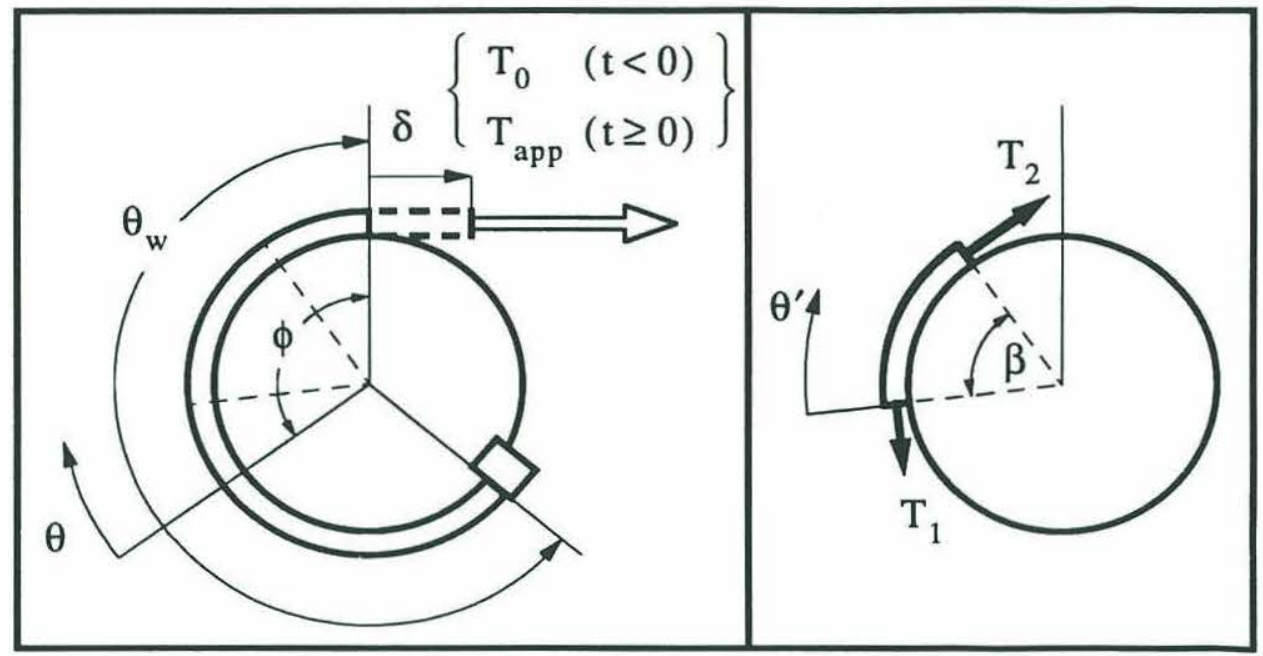

Figure 2.6. Schematic of Wrapped Cable During Loading

Integrating $\varepsilon_{\mathrm{eq}}$ over the affected region gives us the total elongation of the loaded cable at equilibrium. If the cable material obeys Hooke's law (i.e. is linearly elastic) and we assume the cable load is uniaxial we can rewrite (2-31) as

$$
\mathrm{d} \delta=\frac{\sigma_{\mathrm{eq}}}{\mathrm{E}} \mathrm{R} \mathrm{d} \theta
$$

where $\sigma_{\text {eq }}$ is the stress associated with the strain $\varepsilon_{\text {eq }}$. If we also assume that the stress is constant in the cable's cross equation (2-33) becomes

$$
\mathrm{d} \delta=\frac{\mathrm{T}(\theta)}{\mathrm{EA}} \mathrm{Rd} \theta
$$

where $T(\theta)$ is the tension in the element and A is the cross sectional area of the cable, which we assume to be constant. In section 2.2.3 we showed that the equilibrium tension varies exponentially from $T_{0}$ to $T_{\text {app }}$ across the affected angle of wrap. We consider now an arc of cable $\beta$ within this region for which the tensions acting on the left and right hand ends are $T_{1}$ and $T_{2}$ respectively. The tension profile in the region $0 \leq \theta^{\prime} \leq \beta$ is

$$
\mathrm{T}\left(\theta^{\prime}\right)=\mathrm{T}_{1} \mathrm{e}^{\mathrm{e}^{*} \theta^{\prime}}
$$

where $\theta^{\prime}$ is measured from the left end of $\beta$ and 


$$
\mu^{*}=\mu \operatorname{sgn}\left(\frac{\mathrm{T}_{\text {app }}}{\mathrm{T}_{0}}-1\right)=\mu \operatorname{sgn}\left(\frac{\mathrm{T}_{2}}{\mathrm{~T}_{1}}-1\right)
$$

Using equations (2-34) and (2-35) and integrating we can write the elongation as

$$
\begin{aligned}
& \int_{0}^{\delta_{e q \beta}} \mathrm{d} \delta=\int_{0}^{\beta} \frac{\mathrm{RT}}{\mathrm{EA}} \mathrm{e}^{\mu^{*} \theta^{\prime}} \mathrm{d} \theta^{\prime} \\
& \delta_{\mathrm{eq} \beta}=\frac{\mathrm{RT}{ }_{1}}{\mathrm{EA} \mu^{*}}\left(\mathrm{e}^{\mu^{*} \beta}-1\right) \\
& \delta_{\mathrm{eq} \beta}=\frac{\mathrm{R}}{\mathrm{EA} \mu^{*}}\left(\mathrm{~T}_{2}-\mathrm{T}_{1}\right)
\end{aligned}
$$

where we have recognized that $T_{1} e^{\mu^{*} \beta}=T(\beta)=T_{2}$. Thus we see that the total elongation (relative to the unstressed length) of an exponentially loaded cable is proportional to the difference between the tensions applied to its ends. (Note that $\mu^{*}$ ensures that $\delta_{\text {eq } \beta}$ is always positive regardless of the relative size of $T_{1}$ and $T_{2}$ ).

To find the initial elongation $\delta_{0 \beta}$ of the cable in $\beta$ we return to equation (2-31) and integrate the $\varepsilon_{0}$ term across $\beta$ to get

$$
\begin{aligned}
\int_{0}^{\delta_{0 \beta}} \mathrm{d} \delta_{0} & =\int_{0}^{\beta} \frac{\mathrm{T}_{0} \mathrm{R}}{\mathrm{EA}} \mathrm{d} \theta^{\prime} \\
\delta_{0 \beta} & =\frac{\mathrm{T}_{0} \mathrm{R}}{\mathrm{EA}} \beta
\end{aligned}
$$

Thus we find the net change in elongation of the cable in $\beta$ by subtracting the initial elongation $\delta_{0 \beta}$ from the equilibrium elongation $\delta_{\text {eq } \beta}$ to get

$$
\delta_{\beta}=\delta_{\text {eq } \beta}-\delta_{0 \beta}
$$




$$
\delta_{\beta}=\frac{\mathrm{R}}{\mathrm{EA}^{*}}\left(\mathrm{~T}_{2}-\mathrm{T}_{1}-\mathrm{T}_{0} \mu^{*} \beta\right)
$$

Equation (2-43) applies to any exponentially loaded arc of cable $\beta$ whose initial tension was uniformly equal to $T_{0}$.

To apply this result to the entire affected angle of wrap we substitute $T_{0}, T_{\text {app }}$ and $\theta_{\text {aff }}$ for $\mathrm{T}_{1}, \mathrm{~T}_{2}$ and $\beta$ to get

$$
\delta=\frac{\mathrm{R}}{\mathrm{EA} \mu^{*}}\left(\mathrm{~T}_{\mathrm{app}}-\mathrm{T}_{0}-\mu^{*} \theta_{\mathrm{aff}}\right)
$$

If $\theta_{\mathrm{w}}>\phi$ we can substitute $\theta_{\text {aff }}=\phi$ which yields

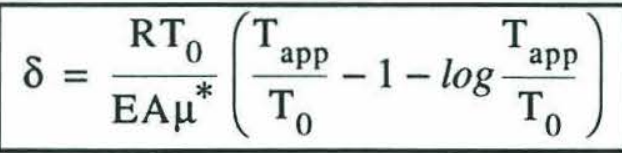

Equation (2-48) gives deflection of a uniformly pretensioned cable when we change the load applied to its free end from $T_{0}$ to $T_{a p p}$. The earliest appearance of this result appears to be that presented by Green [6] in his 1955 publication on continuous flat belt drives.

To find the associated stiffness $k$ we differentiate (2-48) with respect to $T_{\text {app }}$ and rearrange terms to get

$$
\mathrm{k}=\frac{\mathrm{dT}_{\mathrm{app}}}{\mathrm{d} \delta}=\frac{\mathrm{EA} \mu^{*}}{\mathrm{R}}\left(\frac{\mathrm{T}_{\mathrm{app}}}{\mathrm{T}_{\mathrm{app}}-\mathrm{T}_{0}}\right)
$$

We find it convenient to introduce the dimensionless tension $\tau$, defined as

$$
\tau \equiv \frac{\mathrm{T}_{\mathrm{app}}}{\mathrm{T}_{0}}
$$

Making this substitution equations (2-48) and (2-46) become

$$
\delta=\frac{\mathrm{RT}_{0}}{\mathrm{EA \mu}^{*}}(\tau-1-\log \tau)
$$

and 


$$
\mathrm{k}=\frac{\mathrm{EA} \mu^{*}}{\mathrm{R}}\left(\frac{\tau}{\tau-1}\right)
$$

while $\theta_{\text {aff }}$ and $\mu^{*}$ become

$$
\begin{gathered}
\theta_{\mathrm{aff}}=\frac{1}{\mu^{*}} \log \tau \\
\mu^{*}=\mu \operatorname{sgn}(\tau-1) .
\end{gathered}
$$

Figure 2.7. and Figure 2.8. show plots of the normalized elongation and normalized stiffness of the wrapped cable as a function of $\tau$ (recall that $\mu^{*}$ changes sign at $\tau=1$ i.e. when $\mathrm{T}_{\text {app }}=\mathrm{T}_{0}$ ).

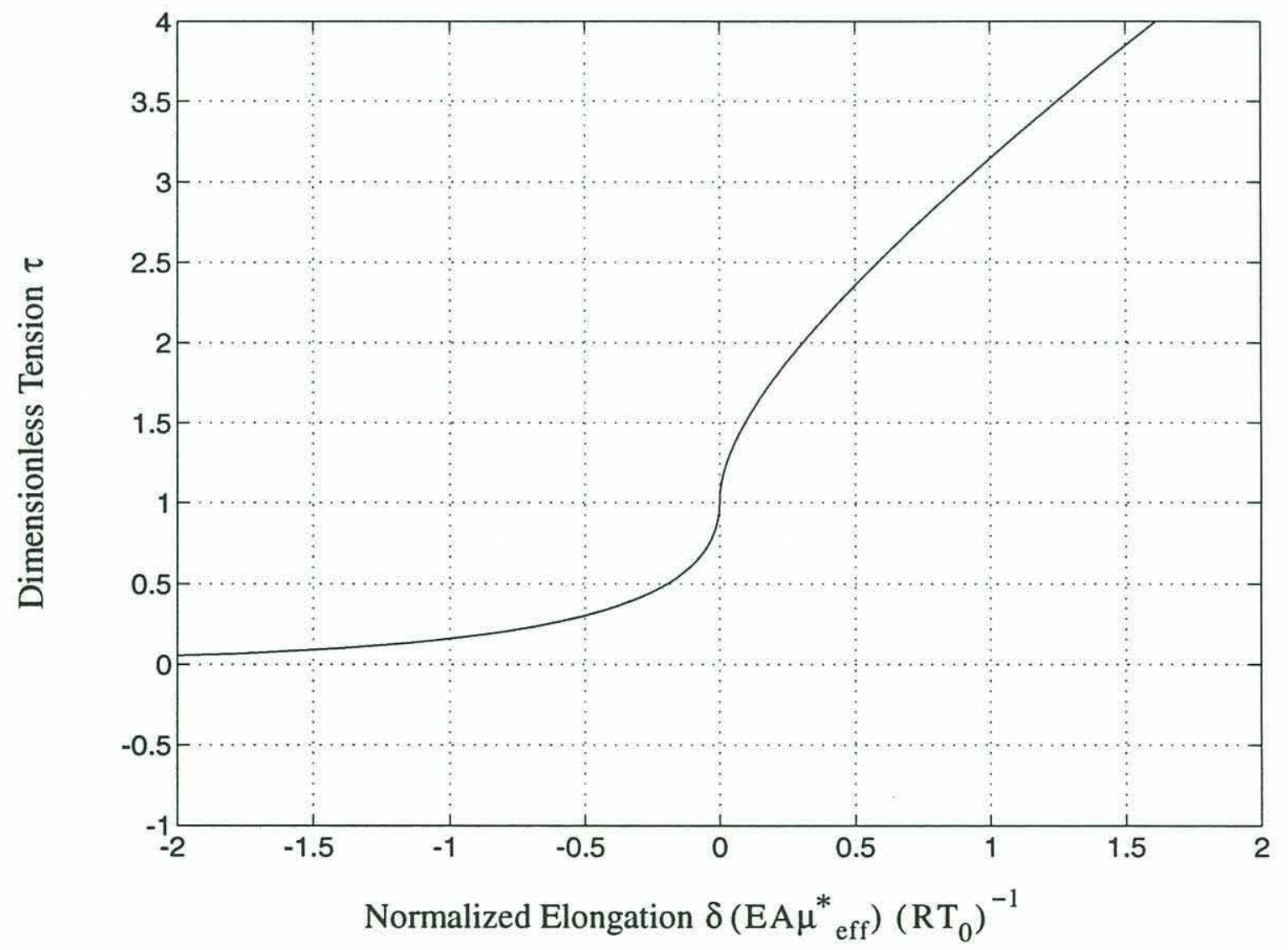

Figure 2.7. Elongation of Wrapped Cable on a Non-rotating Pulley 


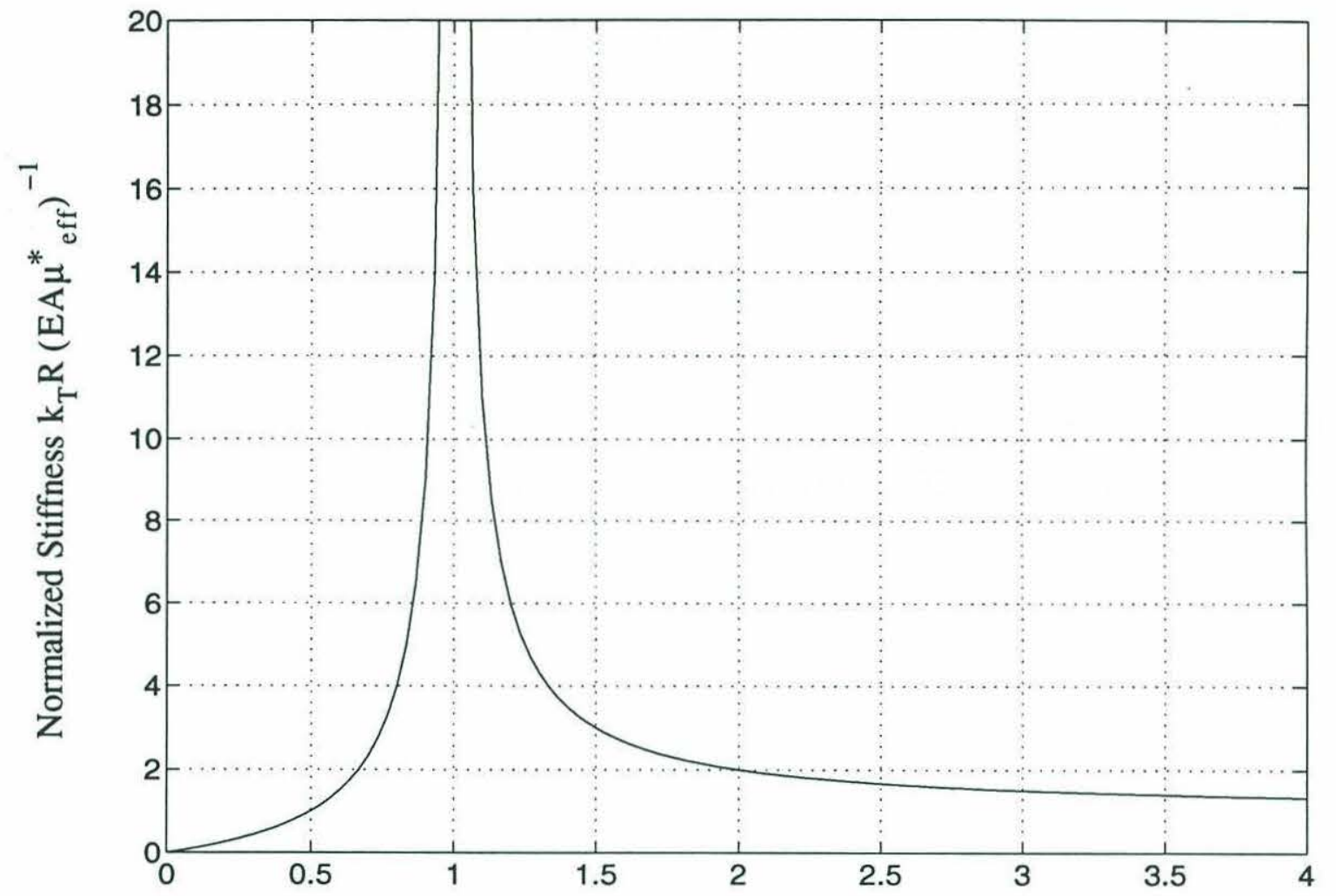

Dimensionless Tension $\tau$

Figure 2.8. Stiffness of Wrapped Cable on a Non-rotating Pulley

(Note: In a real cable transmission we would never have infinite stiffness because there will always be some finite length of cable tangent to the pulley to contribute some compliance).

We note several important characteristics of the cable's response to changes in load.

\section{Softening Spring Behavior.}

Nearly infinite for small changes in load, the stiffness drops off rapidly as the magnitude of the load grows. The stiffness approaches one of two asymptotic values; $\mathrm{k}=\frac{\mathrm{EA} \mu^{*}}{\mathrm{R}}$ if we increase the cable tension or $\mathrm{k}=0$ if we decrease the tension.

2. Dependence of Response on Direction of Load Change.

Although we can increase the tension $\tau$ as much as we like, we can only reduce it by the amount $\mathrm{T}_{0}$ before the cable goes slack. 
As the applied load approaches zero the affected angle of wrap $\theta_{\text {aff }}=\frac{1}{\mu} \log \tau$ approaches infinity. The tension in this region approaches zero resulting in a large deflection as this substantial amount of cable contracts. Increases in tension, however, have much less impact on the growth of $\theta_{\mathrm{aff}}$, resulting in more modest deflections.

\section{Dependence of Stiffness on Pretension $\mathrm{T}_{0}$.}

By substituting $\mathrm{T}_{\text {app }}=\mathrm{T}_{\mathrm{o}}+\Delta \mathrm{T}$ into equation (2-46) we get $\mathrm{k}=\frac{\mathrm{EA} \mu^{*}}{\mathrm{R}}\left(\frac{\mathrm{T}_{0}+\Delta \mathrm{T}}{\Delta \mathrm{T}}\right)$. The two asymptotic stiffnesses mentioned above are unaffected by $\mathrm{T}_{0}$. For small $\Delta \mathrm{T}$, however, we get $k \approx \frac{E A \mu^{*}}{R}\left(\frac{T_{0}}{\Delta T}\right)$ and we see that the stiffness is roughly proportional to the pretension $T_{0}$.

\subsubsection{Elongation During Unloading}

Referring to Figure 2.9. we assume that the load $\mathrm{T}_{\text {app }}$ applied to the cable has been changed from the initial uniform value $\mathrm{T}_{0}$ to some peak value $\mathrm{T}_{\mathrm{pk}}$ and allowed to reach equilibrium so that

$$
T(\theta)=T_{0} e^{\mu^{*} \theta}
$$

and

$$
\theta_{\text {aff }}=\frac{1}{\mu^{*}} \log \frac{\mathrm{T}_{\mathrm{pk}}}{\mathrm{T}_{0}}
$$

where

$$
\mu^{*}=\mu \operatorname{sgn}\left(\frac{\mathrm{T}_{\mathrm{pk}}}{\mathrm{T}_{0}}-1\right)
$$

At some time $t_{1}$ we return the applied load to some intermediate value $T_{\text {app }}$ which lies 
between $T_{0}$ and $T_{p k}$. We wish to find the equilibrium elongation $\delta$ associated with this loading sequence. To do so we follow the same steps used to find the elongation during loading; identify the region of cable affected by the change in applied load, determine the new tensile profile, and use this profile to find the change in elongation.

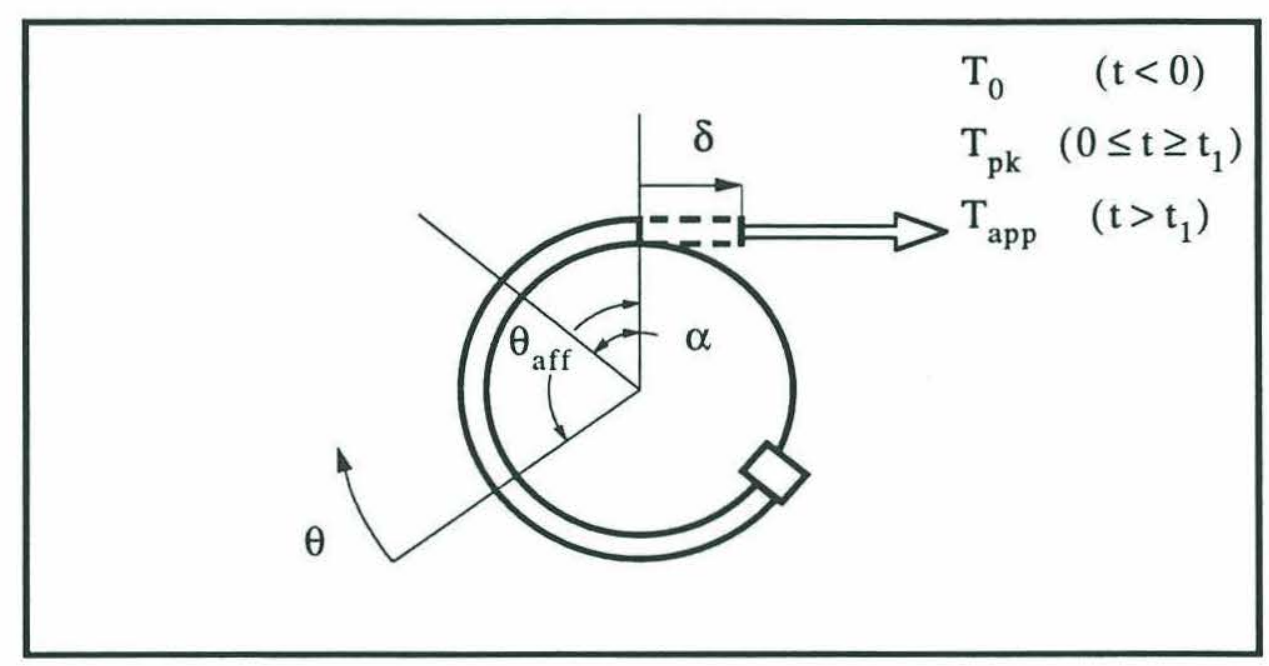

Figure 2.9. Schematic of Wrapped Cable During Unloading

\section{Affected Region:}

We define a new affected angle of wrap $\alpha$ defined as the region of cable whose tension changes from the equilibrium profile (2-52) when we reduce the applied load from its peak value. To begin we consider a differential cable element at the cable's departure point as we begin to at the instant we change the load from the peak load $\mathrm{T}_{\mathrm{pk}}$ to $\mathrm{T}_{\mathrm{app}}$. If the element does not slip there will be an abrupt change in tension across it so that $\left|\frac{\mathrm{dT}}{\mathrm{d} \theta}\right|=\infty$. However, applying the differential slip condition (2-11), we see that this element can only be in equilibrium if $\left|\frac{\mathrm{dT}}{\mathrm{d} \theta}\right| \leq \mu \mathrm{T}_{\mathrm{pk}}$. Therefore the element must slip and begins to do so the instant the load starts to change, initiating the growth of $\alpha$. This region continues to grow until it reaches equilibrium, i.e. when $\alpha$ satisfies the integral slip condition (2-19). Applying (219) we get

$$
\alpha=\frac{1}{\mu^{* *}} \log \frac{\mathrm{T}_{\mathrm{app}}}{\mathrm{T}_{\mathrm{b}}}
$$




$$
\mu^{* *}=\mu \operatorname{sgn}\left(\frac{\mathrm{T}_{\mathrm{app}}}{\mathrm{T}_{\mathrm{b}}}-1\right)
$$

We can show, however, that $\mu^{* *}=-\mu^{*}$. To verify this we consider the two possible loading scenarios. If we initially increase and subsequently decrease the applied load then $\mathrm{T}_{\mathrm{pk}}>\mathrm{T}_{0}$ and $\mathrm{T}_{\mathrm{b}}>\mathrm{T}_{\mathrm{app}}$ which, applying equations (2-54) and (2-56), leads to $\mu^{*}=\mu$ and $\mu^{* *}=-\mu$. An initial decrease and subsequent increase in applied load mean that $\mathrm{T}_{\mathrm{pk}}<\mathrm{T}_{0}$ and $\mathrm{T}_{\mathrm{b}}<\mathrm{T}_{\text {app }}$, in which case $\mu^{*}=-\mu$ and $\mu^{* *}=\mu$. Thus $\mu^{* *}=-\mu^{*}$ holds and we can rewrite equation $(2-55)$ as

$$
\alpha=-\frac{1}{\mu^{*}} \log \frac{T_{\text {app }}}{T_{b}}
$$

By the definition of $\alpha$ the tension $\mathrm{T}_{\mathrm{b}}$ acting on the left hand end is

$$
\mathrm{T}_{\mathrm{b}}=\mathrm{T}\left(\theta_{\mathrm{aff}}-\alpha\right)=\mathrm{T}_{0} \mathrm{e}^{\mu^{*}\left(\theta_{\mathrm{aff}}-\alpha\right)}
$$

where $\theta=\theta_{\text {aff }}-\alpha$ is the location of the left hand side of $\alpha$. By substituting into the equilibrium condition (2-57) we find that the new affected angle of wrap continues to grow until

$$
\alpha=-\frac{1}{\mu^{*}} \log \frac{\mathrm{T}_{\mathrm{app}}}{\mathrm{T}_{0} \mathrm{e}^{\mu\left(\theta_{\text {aff }}-\alpha\right)}}
$$

Rearranging terms we get

$$
\begin{aligned}
\mathrm{e}^{-\mu^{*} \alpha} & =\frac{\mathrm{T}_{\text {app }}}{\mathrm{T}_{0} \mathrm{e}^{\mu^{*}\left(\theta_{\text {aff }}-\alpha\right)}} \\
\frac{\mathrm{T}_{\text {app }}}{\mathrm{T}_{0}} & =\mathrm{e}^{\mu^{*}\left(\theta_{\text {aff }}-2 \alpha\right)}
\end{aligned}
$$

Finally we use equation (2-53) to eliminate $\theta_{\mathrm{aff}}$ and solve for $\alpha$ to get

$$
\alpha=\frac{1}{2 \mu} \log \frac{\mathrm{T}_{\mathrm{pk}}}{\mathrm{T}_{\mathrm{app}}}
$$




\section{Chapter 2: The Mechanics of Wrapped Cable}

This is the portion of $\theta_{\text {aff }}$ affected by changing the applied load from its peak value $T_{p k}$ to the $\mathrm{T}_{\text {app }}$.

\section{Tensile Profile:}

When the cable reaches equilibrium every element in the new affected region $\theta_{\text {aff }}-\alpha \leq \theta \leq \theta_{\text {aff }}$ supports the maximum load that it can withstand without slipping, i.e.

$$
\mathrm{dT}=-\mu^{*} \mathrm{Td} \theta
$$

where the minus sign reflects, as we showed above, that the friction force now acts in the opposite direction of the friction force associated with the original affected angle $\theta_{\text {aff }}$. To find the new tensile profile we rearrange terms and integrate across the region to get

$$
\begin{gathered}
\int_{\mathrm{T}(\theta)}^{\mathrm{T}_{\mathrm{app}}} \frac{\mathrm{dT}}{\mathrm{T}}=\int_{\theta}^{\theta_{\text {aff }}}-\mu^{*} \mathrm{~d} \theta \\
\mathrm{T}(\theta)=\mathrm{T}_{\text {app }} \mathrm{e}^{\mu^{*}\left(\theta_{\text {aff }}-\theta\right)}
\end{gathered}
$$

Eliminating $\theta_{\text {aff }}$ the tensile profile $\alpha$ becomes

$$
\mathrm{T}(\theta)=\frac{\mathrm{T}_{\mathrm{app}} \mathrm{T}_{\mathrm{pk}}}{\mathrm{T}_{0}} \mathrm{e}^{-\mu^{*} \theta}
$$

Thus the tensile profile over the original affected region is

$$
\mathrm{T}(\theta)=\left[\begin{array}{cc}
\mathrm{T}_{\mathrm{o}} \mathrm{e}^{\mu \theta} & {\left[0 \leq \theta \leq\left(\theta_{\mathrm{aff}}-\alpha\right)\right]} \\
\frac{\mathrm{T}_{\mathrm{app}} \mathrm{T}_{\mathrm{pk}}}{\mathrm{T}_{0}} \mathrm{e}^{-\mu \theta} & {\left[\left(\theta_{\mathrm{aff}}-\alpha\right) \leq \theta \leq \theta_{\mathrm{aff}}\right]}
\end{array}\right]
$$

To find the tension $T_{b}$ at the boundary between the two affected regions we substitute $\theta=\theta_{\text {aff }}-\alpha$ into either equation (2-65) or (2-66) to get

$$
\mathrm{T}_{\mathrm{b}}=\sqrt{\mathrm{T}_{\mathrm{app}} \mathrm{T}_{\mathrm{pk}}}
$$

Thus $T_{b}$ equals the geometric mean of the peak load $T_{p k}$ and the present load $T_{a p p}$. 


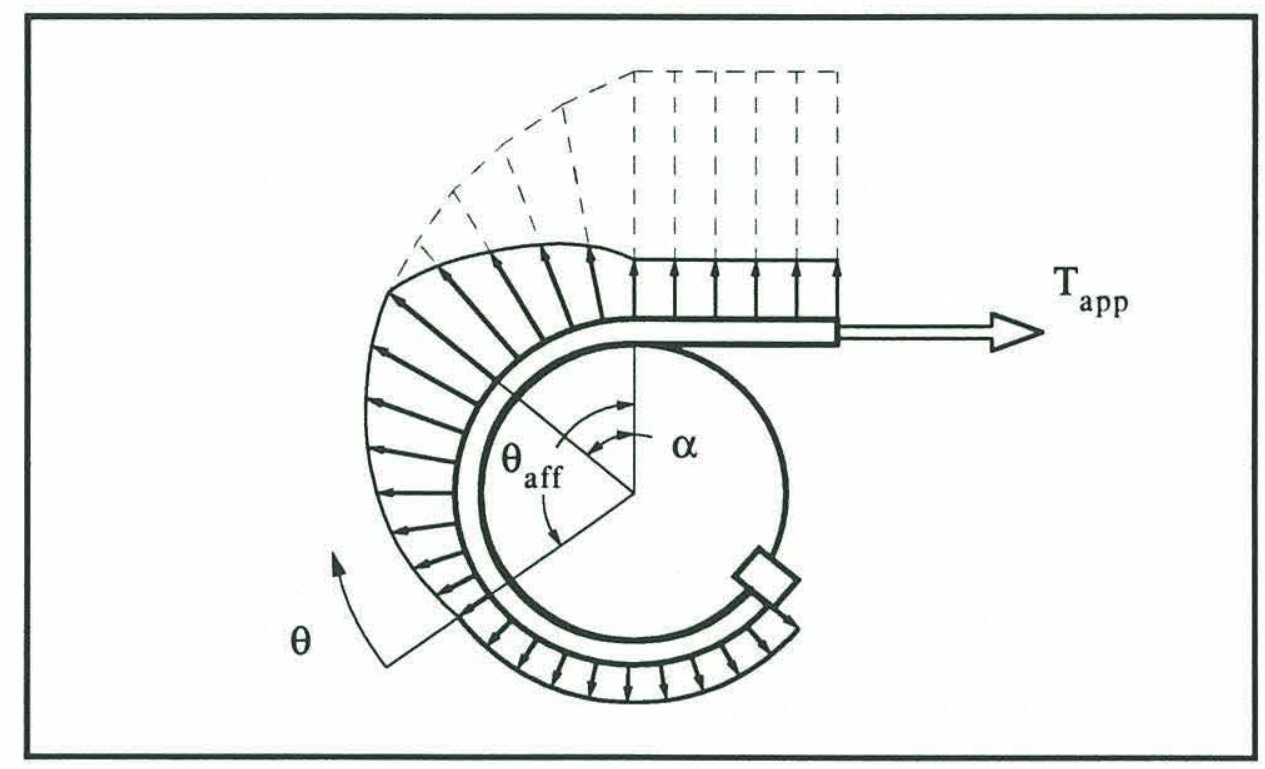

\section{Figure 2.10. Tensile Profile During Unloading}

Figure 2.10. shows the tension profile when we increase (i.e. $\mathrm{T}_{\mathrm{pk}}>\mathrm{T}_{0}$ ) and subsequently decrease the applied load $\mathrm{T}_{\mathrm{app}}$. Friction, capable of supporting a tension difference in either direction, traps a record of the cable's load history so that the wrapped cable becomes a sort of mechanical memory device. We call the profile shown in Figure 2.10. a tension bump. If we had initially reduced and then increased the tension $\mathrm{T}_{\mathrm{app}}$ (i.e. if $\mathrm{T}_{\mathrm{pk}}<\mathrm{T}_{0}$ ) the tension at $\alpha$ would be lower than the surrounding tension and we would have a tension dip.

To "erase" any knowledge of the previous load $\mathrm{T}_{\mathrm{pk}}$ we decrease $\mathrm{T}_{\text {app }}$ until $\alpha$ grows to equal $\theta_{\text {aff }}$ or

$$
\begin{aligned}
\frac{1}{\mu} \log \frac{\mathrm{T}_{\mathrm{pk}}}{\mathrm{T}_{0}} & =\frac{1}{2 \mu} \log \frac{\mathrm{T}_{\mathrm{pk}}}{\mathrm{T}_{\mathrm{app}}} \\
\frac{\mathrm{T}_{\mathrm{app}}}{\mathrm{T}_{0}} & =\frac{\mathrm{T}_{0}}{\mathrm{~T}_{\mathrm{pk}}}
\end{aligned}
$$

or in dimensionless terms when 


$$
\tau=\frac{1}{\tau_{\mathrm{pk}}}
$$

If we instead keep $\mathrm{T}_{\text {app }}$ constant and rotate the pulley counter clockwise, the tension bump rotates intact with the pulley while new cable wraps onto the pulley with constant tension $\mathrm{T}_{\mathrm{app}}$. If we keep $\mathrm{T}_{\text {app }}$ constant and rotate the pulley clockwise an amount $\beta$, by defining $\theta_{\mathrm{aff}}{ }^{\prime}=\theta_{\mathrm{aff}}-\beta$ and substituting for $\theta_{\mathrm{aff}}$ in equation (2-61) we find the new tension bump location $\alpha^{\prime}$ is

$$
\alpha^{\prime}=\alpha_{\text {app }}-\frac{\beta}{2}
$$

where $\alpha_{\text {app }}$ is the bump location before we rotated the pulley. To "erase" any knowledge of the original load $\mathrm{T}_{\mathrm{pk}}$ we must turn the pulley until $\theta_{\mathrm{aff}}{ }^{\prime}=\alpha^{\prime}$ or

$$
\theta_{\mathrm{aff}}-\beta=\alpha_{\mathrm{app}}-\frac{\beta}{2}
$$

which becomes

$$
\beta=2\left(\theta_{\text {aff }}-\alpha_{\text {app }}\right)=\frac{1}{\mu} \log \frac{\mathrm{T}_{\mathrm{app}} \mathrm{T}_{\mathrm{pk}}}{\mathrm{T}_{0}^{2}}
$$

\section{Elongation:}

To find the elongation associated with this tension bump we add the elongations associated with each of the two regions $0 \leq \theta \leq\left(\theta_{\text {aff }}-\alpha\right)$ and $\alpha \leq \theta \leq \theta_{\text {aff }}$. Applying equation (2$43)$ to each region we find that the elongations of the two sections are

$$
\begin{aligned}
\delta_{\alpha} & =\frac{\mathrm{R}}{\mathrm{EA} \mu^{*}}\left(\mathrm{~T}_{\mathrm{b}}-\mathrm{T}_{\mathrm{app}}-\mu^{*} \mathrm{~T}_{0} \alpha\right) \\
\delta_{\left(\theta_{\mathrm{aff}}-\alpha\right)} & =\frac{\mathrm{R}}{\mathrm{EA} \mu^{*}}\left(\mathrm{~T}_{\mathrm{b}}-\mathrm{T}_{0}-\mu^{*} \mathrm{~T}_{0}\left(\theta_{\mathrm{aff}}-\alpha\right)\right)
\end{aligned}
$$

Adding these we find that the elongation of the tension bump relative to the its initial uniformly pretensioned state to be 


$$
\delta=\frac{\mathrm{R}}{\mathrm{EA} \mu^{*}}\left(2 \mathrm{~T}_{\mathrm{b}}-\mathrm{T}_{0}-\mathrm{T}_{\mathrm{app}}-\mu^{*} \mathrm{~T}_{0} \theta_{\mathrm{aff}}\right)
$$

Substituting for $\mathrm{T}_{\mathrm{b}}$ and $\theta_{\mathrm{aff}}$ we get

$$
\delta=\frac{\mathrm{R}}{\mathrm{EA \mu}} \mu^{*}\left(2 \sqrt{\mathrm{T}_{\mathrm{app}} \mathrm{T}_{\mathrm{pk}}}-\mathrm{T}_{0}-\mathrm{T}_{\mathrm{app}}-\mathrm{T}_{0} \log \frac{\mathrm{T}_{\mathrm{pk}}}{\mathrm{T}_{0}}\right)
$$

or, in terms of the dimensionless tension $\tau$, this becomes

$$
\delta=\frac{\mathrm{RT}_{0}}{\mathrm{EA}^{*}}\left(2 \sqrt{\tau \tau_{\mathrm{pk}}}-1-\tau-\log \tau_{\mathrm{pk}}\right)
$$

\subsubsection{Energy Dissipated During Loading}

Because of friction the stretching cable dissipates energy as it slips against the pulley. Referring to Figure 2.6. the work done by friction at a position $\theta$ is

$$
\mathrm{dW}=\mathrm{F}_{\mathrm{fr}}(\theta) \delta(\theta)
$$

where $\mathrm{F}_{\mathrm{fr}}(\theta)$ is the frictional force at $\theta$ and $\delta(\theta)$ is the total length of cable that slides past this point as the system approaches equilibrium. We showed in section 2.2.1 that

$$
\mathrm{F}_{\mathrm{fr}}(\theta)=\mu^{*} \mathrm{~T}(\theta) \mathrm{d} \theta
$$

By substituting the equilibrium tensile profile $\mathrm{T}(\theta)=\mathrm{T}_{0} \mathrm{e}^{\mu^{*} \theta}$ this becomes

$$
\mathrm{F}_{\mathrm{fr}}(\theta)=\mu^{*} \mathrm{~T}_{0} \mathrm{e}^{\mu^{*} \theta} \mathrm{d} \theta
$$

To find the elongation at position $\theta$ we apply equation (2-43) to the arc of cable subtended by $\theta$ to get

$$
\delta(\theta)=\frac{\mathrm{R}}{\mathrm{EA} \mu^{*}}\left(\mathrm{~T}_{0} \mathrm{e}^{\mathrm{\mu}^{*} \theta}-\mathrm{T}_{0}-\mathrm{T}_{0} \mu^{*} \theta\right)
$$

Eliminating $\mathrm{F}_{\mathrm{fr}}(\theta)$ and $\delta(\theta)$ from equation (2-80) we get 


$$
\mathrm{dW}=\left(\mu^{*} \mathrm{~T}_{0} \mathrm{e}^{\mu^{*} \theta} \mathrm{d} \theta\right) \frac{\mathrm{T}_{0} \mathrm{R}}{\mathrm{EA} \mu^{*}}\left(\mathrm{e}^{\mu^{*} \theta}-1-\mu^{*} \theta\right)
$$

By integrating over the region $0 \leq \theta<\theta_{\text {aff }}$ we find the total energy dissipated during elongation.

$$
\int_{0}^{\mathrm{W}_{\mathrm{d}}} \mathrm{dW}=\frac{\mathrm{T}_{0}^{2} \mathrm{R}}{\mathrm{EA}} \int_{0}^{\theta_{\text {aff }}}\left(\mathrm{e}^{2 \mu^{*} \theta}-\mathrm{e}^{\mu^{*} \theta}-\mu^{*} \theta \mathrm{e}^{\mu^{*} \theta}\right) d \theta
$$

Noting that the last term in parentheses requires integration by parts this becomes

$$
\begin{aligned}
\mathrm{W}_{\mathrm{diss}} & =\left.\frac{\mathrm{T}_{0}^{2} \mathrm{R}}{\mathrm{EA} \mu^{*}}\left(\frac{1}{2} \mathrm{e}^{2 \mu^{*} \theta}-\mathrm{e}^{\mu^{*} \theta}-\left(\mu^{*} \theta \mathrm{e}^{\mu^{*} \theta}-\mathrm{e}^{\mu^{*} \theta}\right)\right)\right|_{0} ^{\theta_{\text {aff }}} \\
& =\left.\frac{\mathrm{T}_{0}^{2} \mathrm{R}}{\mathrm{EA} \mu^{*}}\left(\frac{1}{2} \mathrm{e}^{2 \mu^{*} \theta}-\mu^{*} \theta \mathrm{e}^{\mu^{*} \theta}\right)\right|_{0} ^{\theta_{\text {aff }}}
\end{aligned}
$$

If $\theta_{w}>\phi$ equation (2-24) tells us that $\theta_{\text {aff }}=\frac{1}{\mu^{*}} \log \tau$. Making this substitution we find that the energy dissipated by stretching the cable is

$$
\mathrm{W}_{\mathrm{diss}}=\frac{\mathrm{T}_{0}^{2} \mathrm{R}}{\mathrm{EA} \mu^{*}}\left(\frac{1}{2} \tau^{2}-\tau \log \tau-\frac{1}{2}\right)
$$

\subsubsection{Energy Stored During Loading}

Additional work, stored as strain energy in the elongated cable, is required to change the length of the affected cable. The work dW done in stretching a differential element of wrapped cable is

$$
\mathrm{dW}=\mathrm{T}(\theta) \mathrm{d} \delta(\theta)
$$

where $T(\theta)$ is the equilibrium tension in the element and $d \delta(\theta)$ is the amount by which its length changes in response to the applied load (we assume that the change in tension across the element is much much smaller than the tension $\mathrm{T}(\theta)$ itself). Using equations (2-30) and $(2-31)$ to eliminate $T(\theta)$ and $d \delta(\theta)$ we can rewrite (2-88) as 


$$
d W=\left(T_{0} e^{\mu^{*} \theta}\right) \frac{R}{E A}\left(T_{0} e^{\mu^{*} \theta}-T_{0}\right) d \theta
$$

where we have also made the appropriate substitutions for $\varepsilon_{\mathrm{eq}}$ and $\varepsilon_{0}$ in equation (2-31). We find the work required to stretch the entire affected angle of cable by integrating over the region $0 \leq \theta \leq \theta_{\text {aff }}$.

$$
\begin{gathered}
\int_{0}^{W_{\text {stored }}} d W=\frac{T_{0}^{2} R}{E A} \int_{0}^{\theta_{\text {aff }}}\left(e^{2 \mu^{*} \theta}-e^{\mu^{*} \theta}\right) d \theta \\
W_{\text {stored }}=\frac{T_{0}^{2} R}{E A \mu^{*}}\left(\frac{1}{2} e^{2 \mu^{*} \theta_{\text {aff }}}-e^{\mu^{*} \theta_{\text {aff }}}+\frac{1}{2}\right)
\end{gathered}
$$

Again, if the wrap angle $\theta_{w}>\phi$ equation (2-24) tells us that $\theta_{\text {aff }}=\frac{1}{\mu^{*}} \log (\tau)$ and we get

$$
\mathrm{W}_{\text {stored }}=\frac{\mathrm{T}_{0}^{2} \mathrm{R}}{\mathrm{EA} \mu^{*}}\left(\frac{1}{2} \tau^{2}-\tau+\frac{1}{2}\right)
$$

or

$$
\mathrm{W}_{\text {stored }}=\frac{\mathrm{T}_{0}^{2} \mathrm{R}}{2 \mathrm{EA} \mu^{*}}(\tau-1)^{2}
$$

\subsection{Consistency Checks}

\subsubsection{Conservation of Energy}

If our results are correct the work done by the by the applied load during elongation should equal the sum of the dissipated and stored energies or

$$
\mathrm{W}_{\text {in }}=\mathrm{W}_{\text {diss }}+\mathrm{W}_{\text {stored }}
$$

The work done by the applied load is 


$$
\mathrm{W}_{\mathrm{in}}=\int_{0}^{\delta} \mathrm{T}_{\mathrm{app}} \mathrm{dx}=\mathrm{T}_{\mathrm{app}} \delta
$$

where $\delta$ is the equilibrium deflection at the point at which $\mathrm{T}_{\text {app }}$ is applied and we assume $\mathrm{T}_{\text {app }}$ is independent of $\delta$. Using equation (2-48) to replace $\delta$ and rearranging terms we find the work done by the applied load during elongation to be

$$
\mathrm{W}_{\mathrm{in}}=\frac{\mathrm{RT}_{0}^{2}}{\mathrm{EA} \mu}\left(\tau^{2}-\tau-\tau \log \tau\right)
$$

Returning to equation (2-94) we add $\mathrm{W}_{\text {diss }}$ and $\mathrm{W}_{\text {stored }}$ to get

$$
\mathrm{W}_{\text {diss }}+\mathrm{W}_{\text {stored }}=\frac{\mathrm{RT}_{0}^{2}}{\mathrm{EA} \mu}\left[\left(\frac{1}{2} \tau^{2}-\tau \log \tau-\frac{1}{2}\right)+\left(\frac{1}{2} \tau^{2}-\tau+\frac{1}{2}\right)\right]
$$

which simplifies to

$$
\mathrm{W}_{\text {diss }}+\mathrm{W}_{\text {stored }}=\frac{\mathrm{RT}_{0}^{2}}{\mathrm{EA} \mu}\left(\tau^{2}-\tau-\tau \log \tau\right)
$$

Comparing equations (2-96) and (2-98) we see that the models are consistent with the principle of energy conservation.

\subsubsection{Efficiency Limit of Tension Element Drives}

We now show that the results of the previous sections are consistent with the steady state efficiency limit predicted by Green [6], Tordion [13], and Townsend [15]. Green and Townsend determined the power loss by comparing power in to power out. Tordion derived the efficiency limit by determining the frictional power loss due to slip between the belt and pulley but did so by formulating the problem as an analogous compressible fluid flow problem. We also determine the frictional power loss but we use a more classical mechanics based approach employing the models we have developed. 


\section{Problem Statement:}
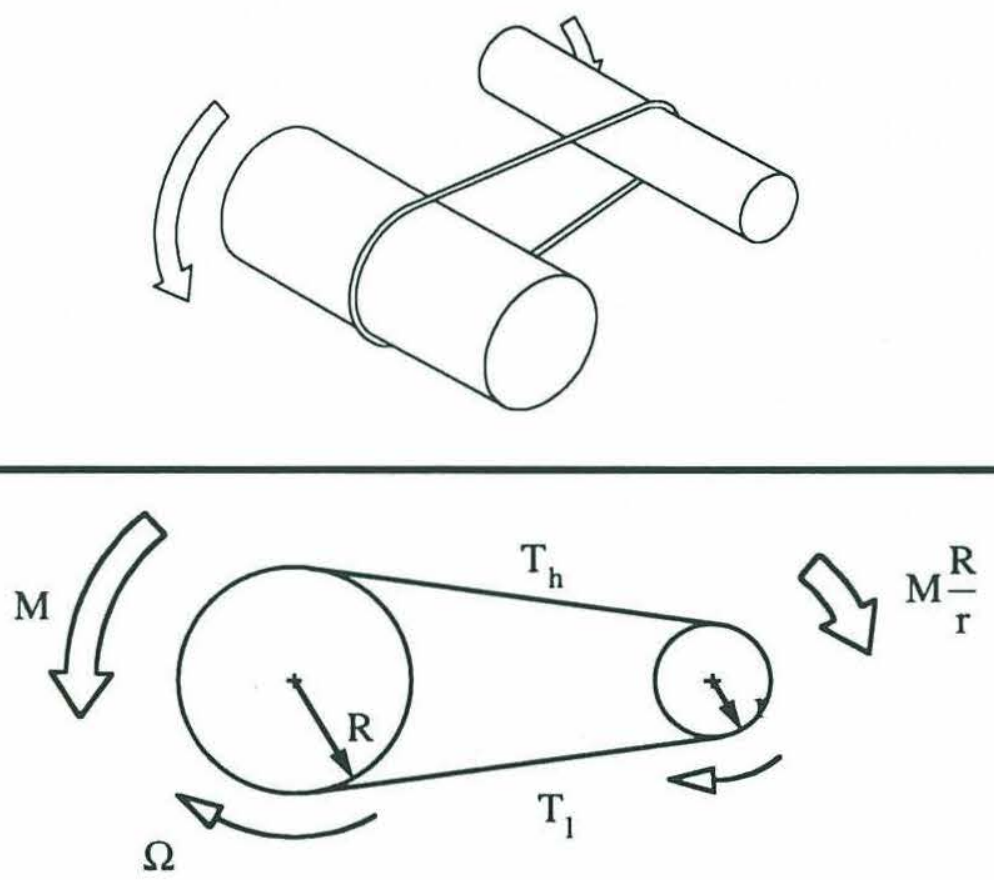

Figure 2.11. Continuously Driven Pulley Under Load

We consider the steady state rotation of the system shown in Figure 2.11 . The pulley $R$ rotates at a constant angular velocity $\Omega$ and the working load $\mathrm{M}$ exactly balances the moment resulting from the difference in cable tensions, i.e. the system is in dynamic equilibrium.

As Reynolds [8] first recognized, the cable on the output pulley R must stretch as it travels from the low tension side to the high tension side and therefore must slip with respect to the pulley surface. Similarly, the cable on input pulley $r$ must also slip, only here it must stretch as the pulley rotates. We wish to determine the continuous power dissipation associated with these regions of slipping cable.

\section{The Affected Angles of Wrap and Tensile Profile}

Swift [12] showed that the affected angle of wrap and the tensile profile in this system are stationary with respect to a non-rotating world frame of reference. To see this we consider the drive initially at rest and uniformly pretensioned to $\mathrm{T}_{0}$. Locking the position of the input pulley $r$ we apply the load $M$ to the output pulley. As a result the cable tensions 
change to $T_{h}$ and $T_{1}$ and two affected angles of wrap develop on each pulley; $\theta_{\text {aff }_{\mathrm{h}}}=\frac{1}{\mu} \log \frac{\mathrm{T}_{\mathrm{h}}}{\mathrm{T}_{0}}$ on the upper halves and $\theta_{\text {aff }_{1}}=\frac{1}{\mu} \log \frac{\mathrm{T}_{0}}{\mathrm{~T}_{1}}$ on the lower halves (see Figure 2.12.a. For clarity we have assumed that the affected regions do not overlap, i.e. that they are still separated by an arc of cable at tension $\mathrm{T}_{0}$ ).

Maintaining the applied load we slowly rotate the input pulley $\mathrm{r}$ in the clockwise direction, thus tending to decrease $\theta_{\mathrm{aff}_{\mathrm{h}}}$ and increase $\theta_{\mathrm{aff}_{1}}$ on the output pulley $\mathrm{R}$. However, (still referring to the output pulley) the loads acting on the ends of $\theta_{\text {aff }}$ are still $\mathrm{T}_{0}$ and $\mathrm{T}_{\mathrm{h}}$ so by the slip condition $\theta_{\text {aff }_{\mathrm{h}}}$ must still equal $\frac{1}{\mu} \log \frac{\mathrm{T}_{\mathrm{h}}}{\mathrm{T}_{0}} \cdot \theta_{\mathrm{aff}_{\mathrm{f}}}$, on the other hand, does in fact increase. The original affected region $\theta_{\text {aff }_{1}}=\frac{1}{\mu} \log \frac{\mathrm{T}_{0}}{\mathrm{~T}_{1}}$ rotates intact with the output pulley while new cable wraps on behind it at constant tension $T_{1}$ (Figure 2.12.b.). Eventually this region of constant tension extends into the interior edge of the upper affected angle, after which only the upper affected angle of wrap $\theta_{\text {aff }_{\mathrm{h}}}$ remains, its value now equal to

$$
\theta_{\mathrm{aff}_{\mathrm{h}}}=\frac{1}{\mu} \log \left(\frac{\mathrm{T}_{\mathrm{h}}}{\mathrm{T}_{1}}\right)
$$

(Figure 2.12.c.). During constant rotation the entire arc of cable defined by $\theta_{\mathrm{aff}_{\mathrm{h}}}$ slips continuously so the slope of the tension in this region is $\frac{d T}{d \theta}=\mu \mathrm{T}$. The tensile profile in the affected angle of wrap is therefore

$$
\mathrm{T}(\theta)=\mathrm{T}_{1} \mathrm{e}^{\mu \theta}
$$

whereas the tension in the remainder of the wrapped cable is uniformly equal to $T_{1}$. Thus $T_{1}$ becomes the effective pretension for the wrapped cable. The development of the input pulley's tensile profile is similar except that $T_{h}$ becomes the effective pretension and only the lower affected region remains, equal to $\theta_{\mathrm{aff}_{1}}=\theta_{\mathrm{aff}_{\mathrm{h}}}=\frac{1}{\mu} \log \left(\frac{\mathrm{T}_{\mathrm{h}}}{\mathrm{T}_{1}}\right)$ 


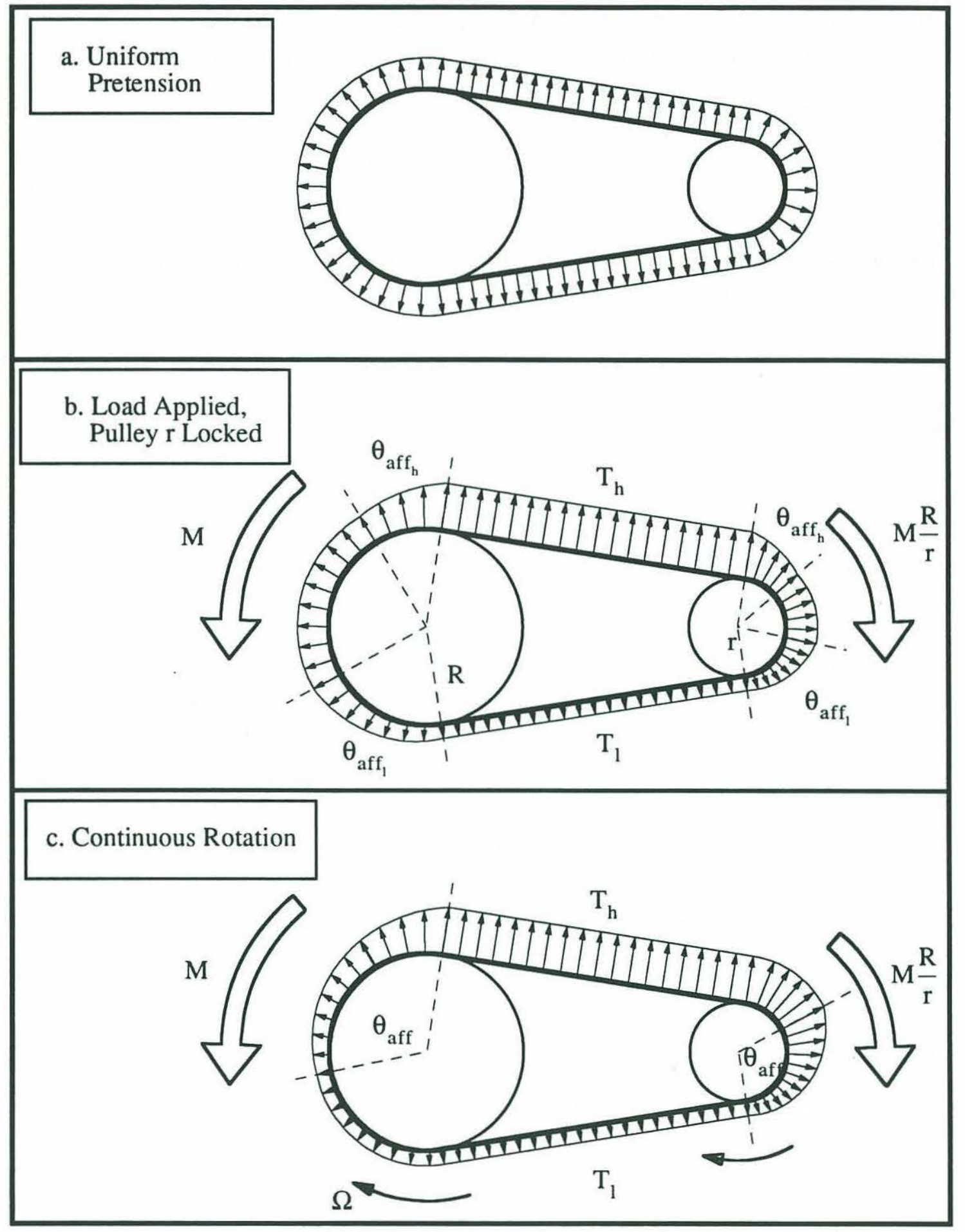

Figure 2.12. Affected Angles of Wrap and Tensile Profiles 


\section{Power Dissipation at the Output Pulley R}

Power dissipation only occurs where cable slips with respect to the pulley (i.e. in the region $0 \leq \theta \leq \theta_{\text {aff }_{\mathrm{h}}}$ for the output pulley $\mathrm{R}$ ). The differential power loss at a position $\theta$ equals the product of the frictional force and the slip velocity or

$$
\mathrm{dP}(\theta)=\mathrm{F}_{\mathrm{fr}}(\theta) \frac{\mathrm{d} \delta(\theta)}{\mathrm{dt}}
$$

where we recognize that the slip velocity is the time derivative of the elongation $\delta(\theta)$. From the derivation of the slip condition in section 2.2.1 we know that

$$
F_{f r}(\theta)=\mu T(\theta) d \theta
$$

Substituting for $\mathrm{T}(\theta)$ we get

$$
\mathrm{F}_{\mathrm{fr}}(\theta)=\mu \mathrm{T}_{1} \mathrm{e}^{\mu \theta} \mathrm{d \theta}
$$

This is the frictional force at the position $\theta$ which again is measured with respect to the stationary world frame.

Our expression for elongation of a wrapped cable gives the amount of slip at a point as a function of $\theta$. By the chain rule we may write the slip velocity $\frac{\mathrm{d} \delta}{\mathrm{dt}}$ as

$$
\frac{\mathrm{d} \delta}{\mathrm{dt}}=\frac{\mathrm{d} \delta}{\mathrm{d} \theta} \frac{\mathrm{d} \theta}{\mathrm{dt}}
$$

To find $\frac{\mathrm{d} \delta}{\mathrm{d} \theta}$ we apply equation (2-48) to the arc of cable subtended by $\theta$ which yields

$$
\delta(\theta)=\frac{\mathrm{R}}{\mathrm{EA \mu}}\left(\mathrm{T}_{1} \mathrm{e}^{\mu \theta}-\mathrm{T}_{1}-\mathrm{T}_{1} \mu \theta\right)
$$

and then differentiate with respect to $\theta$ to get

$$
\frac{\mathrm{d} \delta}{\mathrm{d} \theta}=\frac{\mathrm{RT}_{1}}{\mathrm{EA}}\left(\mathrm{e}^{\mu \theta}-1\right)
$$

Recognizing that $\frac{\mathrm{d} \theta}{\mathrm{dt}}=\Omega$ equation (2-104) becomes 


$$
\frac{\mathrm{d} \delta}{\mathrm{dt}}=\frac{\Omega \mathrm{RT}_{1}}{\mathrm{EA}}\left(\mathrm{e}^{\mu \theta}-1\right)
$$

Substituting for $\frac{\mathrm{d} \delta}{\mathrm{dt}}$ and $\mathrm{F}_{\mathrm{fr}}$ in $\mathrm{dP}(\theta)$ we get

$$
\mathrm{dP}(\theta)=\left(\mu \mathrm{T}_{1} \mathrm{e}^{\mu \theta} \mathrm{d} \theta\right)\left[\frac{\Omega \mathrm{RT} \mathrm{T}_{1}}{\mathrm{EA}}\left(\mathrm{e}^{\mu \theta}-1\right)\right]
$$

To find the total power dissipation for the output pulley we integrate over the entire affected region to get

$$
\int_{0}^{\mathrm{P}_{\mathrm{L}_{\mathrm{R}}}} \mathrm{dP}(\theta)=\int_{0}^{\theta_{\mathrm{arf}_{\mathrm{h}}}} \frac{\mu \Omega \mathrm{RT}_{1}^{2}}{\mathrm{EA}}\left(\mathrm{e}^{2 \mu \theta}-\mathrm{e}^{\mu \theta}\right) \mathrm{d} \theta
$$

(Note that the power loss at $\theta=0$ is zero since there is no slip at this point).Thus the power loss at the output pulley $P_{L_{R}}$ is

$$
\begin{gathered}
\mathrm{P}_{\mathrm{L}_{\mathrm{R}}}=\frac{\mu \Omega R \mathrm{~T}_{1}^{2}}{2 \mathrm{EA}}\left(\mathrm{e}^{2 \mu \theta_{\text {aff }}}-2 \mathrm{e}^{\mu \theta_{\text {aff }}}+1\right) \\
\mathrm{P}_{\mathrm{L}_{\mathrm{R}}}=\frac{\mu \Omega R \mathrm{~T}_{1}^{2}}{2 \mathrm{EA}}\left(\mathrm{e}^{\mu \theta_{\text {aff }}}+1\right)^{2}
\end{gathered}
$$

If we substitute $\theta_{\mathrm{aff}_{\mathrm{h}}}=\frac{1}{\mu} \log \left(\frac{\mathrm{T}_{\mathrm{h}}}{\mathrm{T}_{1}}\right)$ and rearrange terms we get

$$
\mathrm{P}_{\mathrm{L}_{\mathrm{R}}}=\frac{\Omega \mathrm{R}}{2 \mathrm{EA}}\left(\mathrm{T}_{\mathrm{h}}-\mathrm{T}_{1}\right)^{2}
$$

Substituting $M=\left(T_{h}-T_{1}\right) R$ we find that power loss at the output pulley $R$ is

$$
\mathrm{P}_{\mathrm{L}_{\mathrm{R}}}=\frac{\Omega \mathrm{M}}{2 \mathrm{EA}}\left(\mathrm{T}_{\mathrm{h}}-\mathrm{T}_{1}\right)
$$

This is the same power loss term that Townsend [15] obtained via a control volume anal- 
ysis. Noting that $\Omega \mathrm{M}$ is the power delivered to the load we can rewrite equation (2-115) as

$$
P_{L_{R}}=P_{\text {out }} \frac{T_{h}-T_{1}}{2 E A}
$$

\section{Overall Efficiency of the Complete Drive}

A similar power loss takes place at the input pulley, the only difference being that the cable contracts as it rotates from the high tension side of the pulley to the low tension side. Performing the same analysis on the input pulley we find its power loss to be the same as that of the output pulley, i.e.

$$
P_{L_{r}}=P_{\text {in }} \frac{T_{h}-T_{1}}{2 E A}
$$

The power the input pulley delivers to the cables must equal the power the output pulley takes from the cables, i.e.

$$
P_{\text {in }}\left(1-\frac{T_{h}-T_{1}}{2 E A}\right)=P_{\text {out }}\left(1+\frac{T_{h}-T_{1}}{2 E A}\right)
$$

Solving for the efficiency $\eta=\frac{P_{\text {out }}}{P_{\text {in }}}$ we get

$$
\eta=\frac{1-\frac{\mathrm{T}_{\mathrm{h}}-\mathrm{T}_{1}}{2 \mathrm{EA}}}{1+\frac{\mathrm{T}_{\mathrm{h}}-\mathrm{T}_{1}}{2 \mathrm{EA}}}
$$

When $\frac{T_{h}-T_{1}}{2 E A} \ll 1$ (which holds for most properly designed cable mechanisms) this is approximately equal to

$$
\eta=1-\frac{T_{h}-T_{1}}{E A}=1-\frac{M}{R E A}
$$

which is the efficiency limit obtained by the others. Thus we conclude that our models are consistent with previous work on the efficiency of belt and cable drives. 


\subsection{Conclusions}

In this chapter we modelled the equilibrium elongation of a cable wrapped around a nonrotating pulley, first as we applied a load and then as we removed it. We summarize our findings below.

\section{Characteristics of Wrapped Cable}

1. Wrapped cable exhibits softening spring behavior.

2. Stiffness increases as we increase pretension $\mathrm{T}_{0}$.

3. Exhibits no stick/slip behavior during elongation.

4. $\mu_{\text {dynamic }}$, not $\mu_{\text {static }}$, determines the equilibrium behavior

5. Applied loads only affect a portion of the wrapped cable.

6. Applying and removing a load creates a stable "tension bump" in the tensile profile of the wrapped cable.

7. The elongation associated with a tension bump determines the hysteresis deflection of the drive. 


\section{Chapter 3 Open Circuit Cable/Pulley Drives}

Using the results of the previous chapter we now model the deflection of a single stage open circuit cable drive during both loading and unloading. We show that in both cases the character of the drive's response depends on the value of a dimensionless parameter we call the geometry-friction number GF. High GF values correspond to a low but relatively linear stiffness whereas low GF values represent much stiffer drives whose stiffness drops off rapidly as we increase the applied load. We also find that for low and moderate GF values increasing the pretension $T_{0}$ increases the stiffness. We end by presenting experimental results which confirm the validity of both the loading and unloading models. 


\subsection{The Open Circuit Cable Drive}

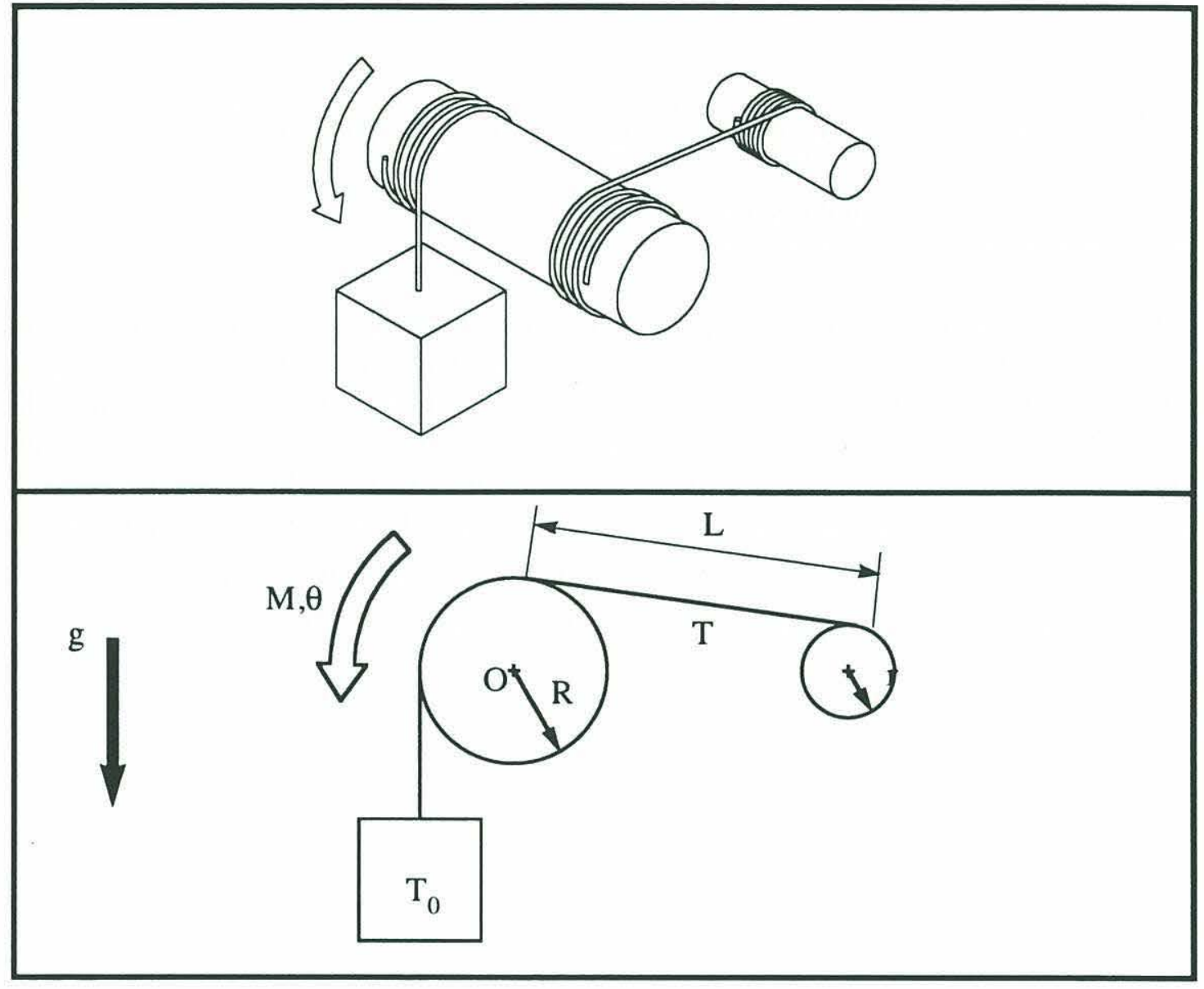

Figure 3.1. Single Stage Pretensioned Open Circuit Drive

Definition: An open circuit cable drive is a cable drive whose cables are only in tension while the drive transmits a load between the input and the output.

Figure 3.1. shows a single stage open circuit cable drive in which a length of cable couples the rotation of the input pulley $r$ and the output pulley $R$ (the ends of the cable are rigidly attached to each pulley). Prior to applying the moment $M$ we turn the input pulley $r$ back and forth through the full range of motion permitted by the drive cable. This ensures that the drive is uniformly pretensioned, i.e. that the cable tension is everywhere equal to $\mathrm{T}_{0}$, the weight of the block. Locking the position of the input pulley $r$ we wish to determine the equilibrium deflection $\theta$ of the output pulley $\mathrm{R}$ when we apply and subsequently remove a moment $\mathrm{M}$. 


\subsection{Modelling}

We find the deflection of the drive by identifying and then simultaneously satisfying the system's geometric, constitutive and equilibrium constraints. The geometric constraint relates pulley deflection $\theta$ to the cable elongation, the constitutive relation relates this elongation to the tension $\mathrm{T}$ in the cable's free length and the equilibrium constraint relates this tension to the applied moment M. In these derivations we assume that

1. the affected angles of wrap $\theta_{\mathrm{aff}}$ on the pulleys are always smaller than the actual angles of wrap

2. the deflection angle $\theta$ is much smaller than the affected angle of wrap $\theta_{\mathrm{aff}}$.

\section{Geometric Constraint:}

The rotation $\theta$ of the output pulley is equal to the cable elongation $\delta$ divided by the pulley radius $\mathrm{R}$ or

$$
\theta=\frac{\delta}{\mathrm{R}}
$$

\section{Equilibrium Constraint:}

At equilibrium the moments acting on pulley $\mathrm{R}$ sum to zero, resulting in

$$
\mathrm{T}=\mathrm{T}_{0}+\frac{\mathrm{M}}{\mathrm{R}}
$$

Dividing by $\mathrm{T}_{0}$ we obtain the dimensionless equilibrium constraint

$$
\tau=1+\mathrm{m}
$$

where we define the dimensionless tension $\tau \equiv \frac{T}{T_{0}}$ and the dimensionless moment $\mathrm{m} \equiv \frac{\mathrm{M}}{\mathrm{RT}_{0}}$

\section{Constitutive Relations:}

The constitutive relation relates the total cable deformation $\delta$ to the tension $\mathrm{T}$ in the tangent 
length of cable. For the open circuit drive the total cable deformation $\delta$ is

$$
\delta=\delta_{\mathrm{R}}+\delta_{\mathrm{L}}+\delta_{\mathrm{r}}
$$

where $\delta_{\mathrm{r}}$ and $\delta_{\mathrm{R}}$ are the deformations of the cable wrapped on the pulleys $\mathrm{r}$ and $\mathrm{R}$ and $\delta_{\mathrm{L}}$ is the deformation of the tangent segment of cable $\mathrm{L}$. To find $\delta_{\mathrm{L}}$ we simply integrate the change in strain resulting from the change in load to get

$$
\delta_{L}=\int_{0}^{L}\left(\varepsilon-\varepsilon_{0}\right) d x=\frac{L}{E A}\left(T-T_{0}\right)
$$

where $\varepsilon$ is the strain in the cable, $T$ is the equilibrium tension in the tangent length and we have assumed a linearly elastic cable. Note that this relation holds regardless of whether we are applying or removing a load.

The behavior of the wrapped cable, however, depends on whether we are loading or unloading the drive. Consequently we obtain a different constitutive relation for each case. We find the constitutive relation during loading by applying equation (2-48) to find $\delta_{\mathrm{R}}$ and $\delta_{\mathrm{r}}$ and, making the appropriate substitutions in equation (3-4), we get

$$
\delta=\frac{\mathrm{T}_{0} \mathrm{R}}{\mathrm{EA}^{*_{\mathrm{R}}}}(\tau-1-\log \tau)+\frac{\mathrm{T}_{0} \mathrm{~L}}{\mathrm{EA}}(\tau-1)+\frac{\mathrm{T}_{0} \mathrm{r}}{\mathrm{EA}_{\mu_{\mathrm{r}}}^{*}}(\tau-1-\log \tau)
$$

where $\mu_{\mathrm{R}}{ }^{*}=\mu_{\mathrm{R}} \operatorname{sgn}(\tau-1)$ and $\mu_{\mathrm{r}}{ }^{*}=\mu_{\mathrm{r}} \operatorname{sgn}(\tau-1)$ are the signed versions of the coefficients of friction between the cable and each of the pulleys. When we collect terms this becomes

$$
\delta=\frac{\mathrm{T}_{0} \mathrm{R}}{\mathrm{EA}}\left(\frac{1}{\mu^{*}{ }_{\mathrm{R}}}+\frac{\mathrm{r}}{\mathrm{R}} \frac{1}{\mu_{\mathrm{r}}^{*}}\right)(\tau-1-\log \tau)+\frac{\mathrm{T}_{0} \mathrm{~L}}{\mathrm{EA}}(\tau-1)
$$

Defining the effective coefficient of friction $\mu^{*}{ }_{\text {eff }}$ as

$$
\mu_{\text {eff }}^{*}=\left(\frac{1}{\mu_{\mathrm{R}}^{*}}+\frac{\mathrm{r}}{\mathrm{R}} \frac{1}{\mu_{\mathrm{r}}^{*}}\right)^{-1}=\left(\frac{1}{\mu_{\mathrm{R}}}+\frac{\mathrm{r}}{\mathrm{R}} \frac{1}{\mu_{\mathrm{r}}}\right)^{-1} \operatorname{sgn}(\tau-1)
$$

the constitutive relation during loading (3-7) becomes 


$$
\delta=\frac{\mathrm{T}_{0} \mathrm{R}}{\mathrm{EA} \mu_{\text {eff }}^{*}}(\tau-1-\log \tau)+\frac{\mathrm{T}_{0} \mathrm{~L}}{\mathrm{EA}}(\tau-1)
$$

(Note: If the coefficients of friction for the two pulleys happen to be the same (i.e. if $\left.\mu_{\mathrm{r}}=\mu_{\mathrm{R}}=\mu\right) \mu_{\text {eff }}^{*}$ simplifies to $\left.\mu_{\text {eff }}^{*}=\frac{\mathrm{R} \mu}{\mathrm{R}+\mathrm{r}} \operatorname{sgn}(\tau-1)\right)$.

To obtain the constitutive relation during unloading we use equation (2-79) to eliminate $\delta_{R}$ and $\delta_{\mathrm{r}}$ from equation (3-4) which yields

$$
\delta=\frac{\mathrm{T}_{0} \mathrm{R}}{\mathrm{EA} \mu^{*}{ }_{\text {eff }}}\left(2 \sqrt{\tau_{\mathrm{pk}}} \tau-1-\tau-\log \tau_{\mathrm{pk}}\right)+\frac{\mathrm{T}_{0} \mathrm{~L}}{\mathrm{EA}}(\tau-1)
$$

By comparing equations (3-9) and (3-10) with equations (2-48) and (2-79) we see that a cable wrapped around two pulleys $R$ and $r$ (with coefficients of friction $\mu_{R}$ and $\mu_{r}$ ) behaves as if it was wrapped around a single pulley $\mathrm{R}$ with an effective coefficient of friction of $\mu^{*}{ }_{\text {eff }}$.

(Note: We have modelled all of the wrapped cable as if it were wrapped on a nonrotating pulley. This presents no problem for cable on pulley $\mathrm{r}$ since we have locked it in place. Pulley R, however, rotates as the cable responds to the applied load and this may cause the affected angle of wrap (and thereby the elongation) to differ from that predicted by the nonrotating pulley model. Nonetheless we can say that any effect will be small if the pulley rotation $\theta$ is very small compared to the affected angle of wrap $\theta_{\text {aff }}$ expected for an equivalently loaded nonrotating pulley, i.e. if $\theta \ll \phi=\frac{1}{\mu^{*}{ }_{\mathrm{R}}} \log \left(\frac{\mathrm{T}}{\mathrm{T}_{0}}\right)$. In section 3.2.4 we verify that typical open circuit drives satisfy this condition and we try to estimate the error for drives which do not satisfy the condition.)

\subsubsection{The Geometry-Friction Number}

We take one last look at the constitutive relations before we solve for the drive's deflection. By combining terms we can rewrite equations (3-9) and (3-10) as

$$
\delta=\frac{\mathrm{T}_{0} \mathrm{R}}{\mathrm{EA} \mu^{*}{ }_{\text {eff }}}\left[\left(1+\frac{\mathrm{L} \mu^{*}{ }_{\text {eff }}}{\mathrm{R}}\right)(\tau-1)-\log (\tau)\right]
$$


and

$$
\delta=\frac{\mathrm{T}_{0} \mathrm{R}}{\mathrm{EA} \mu^{*}{ }_{\text {eff }}}\left[2 \sqrt{\tau_{\mathrm{pk}} \tau}+\left(\frac{\mathrm{L} \mu^{*}{ }_{\text {eff }}}{\mathrm{R}}-1\right)(\tau-1)-2-\log \tau_{\mathrm{pk}}\right]
$$

Note the presence of the dimensionless term $\frac{\mathrm{L \mu}{ }^{*}{ }_{\text {eff }}}{\mathrm{R}}$ in both equation (3-11) and (3-12). This term indicates the relative importance of the elongations of the tangent and wrapped portions of cable. To see this we compare the elongations of the tangent and wrapped portions of cable during loading

$$
\frac{\delta_{\text {tan }}}{\delta_{\text {wrap }}}=\frac{\mathrm{L} \mu_{\text {eff }}^{*}}{\mathrm{R}}\left(1-\frac{\log \tau}{\tau-1}\right)^{-1}
$$

and during unloading

$$
\frac{\delta_{\text {tan }}}{\delta_{\text {wrap }}}=\frac{\mathrm{L} \mu_{\text {eff }}^{*}}{\mathrm{R}}\left(\frac{2 \sqrt{\tau_{\mathrm{pk}} \tau}-2-\log \tau_{\mathrm{pk}}}{(\tau-1)}-1\right)^{-1}
$$

When $\frac{L \mu_{\text {eff }}}{R}$ » 1 (e.g. when the pulleys are far apart) the elongation of the tangent cable dominates and the stiffness is very nearly linear. When $\frac{\mathrm{L} \mu_{\mathrm{eff}}}{\mathrm{R}} \ll 1$ (e.g. when the pulleys nearly touch) the elongation of the wrapped cable dominates and the stiffness is decidedly nonlinear. Thus this parameter determines the character of a drive's response to load. We call this parameter as the Geometry-Friction number and designate it as

$$
\mathrm{GF} \equiv \frac{\mathrm{L} \mu_{\text {eff }}}{\mathrm{R}}
$$

(In the special case of $\mu_{r}=\mu_{R}=\mu$ this hecomes GF $=\frac{L \mu}{R+r}$ ). In general we shall use the signed version of GF defined as GF $\equiv \mathrm{GF} s g n(\tau-1)=\frac{\mathrm{L} \mu^{*}{ }_{\text {eff }}}{\mathrm{R}} \operatorname{sgn}(\mathrm{m})$ where we have used the dimensionless equilibrium constraint (3-3) to replace $\tau-1$ by $\mathrm{m}$. We will encounter the parameter GF in the analysis of every drive investigated in this report. 


\subsubsection{Deflection During Loading}

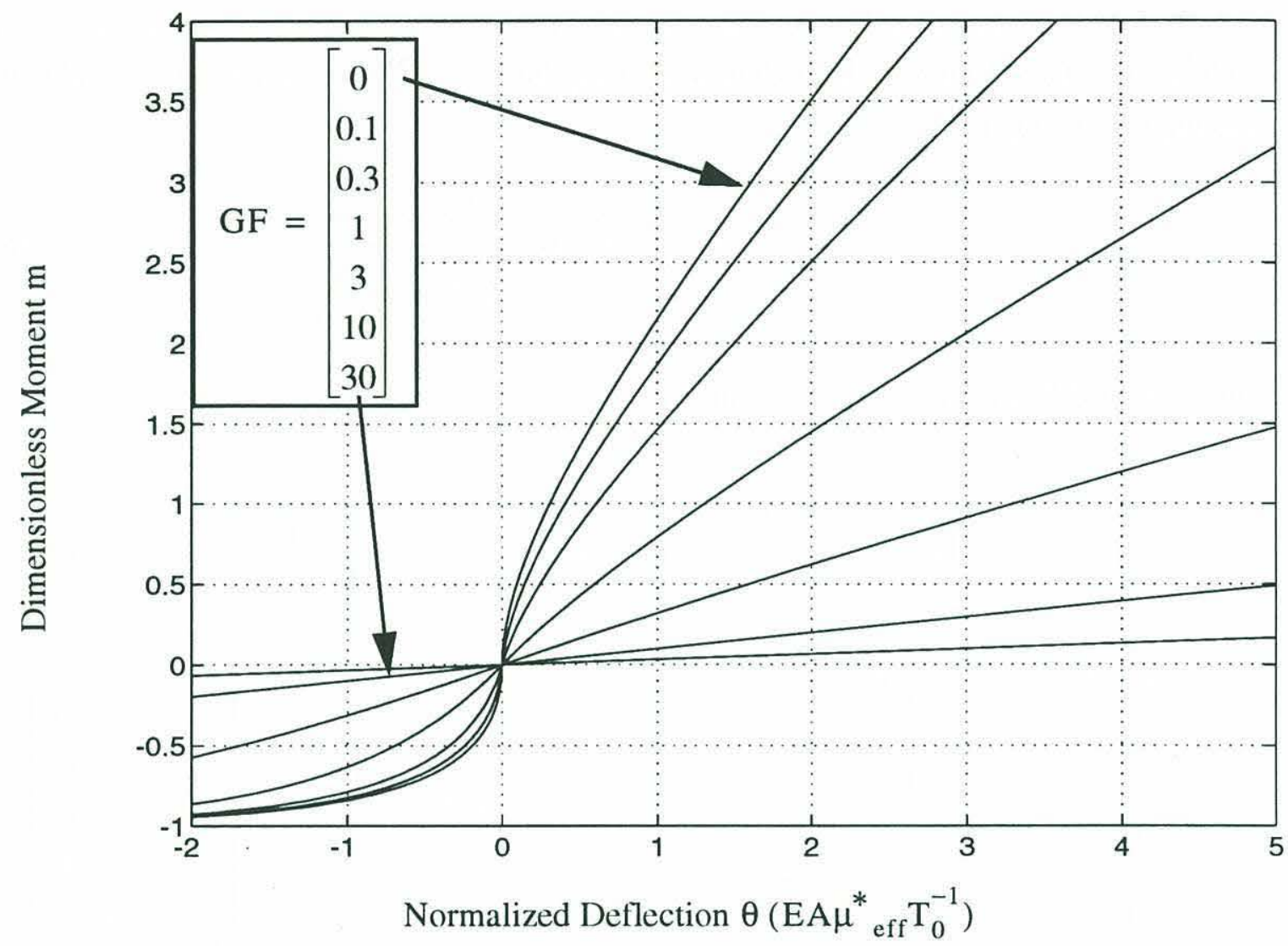

Figure 3.2. Deflection of an Open Circuit Drive During Loading

We now solve for the pulley deflection $\theta$ during loading by substituting the constitutive relation (3-9) and the equilibrium constraint (3-3) into the geometric constraint (3-1) to get

$$
\theta=\frac{\mathrm{T}_{0}}{\mathrm{EA} \mu^{*}{ }_{\text {eff }}}(\mathrm{m}-\log (1+\mathrm{m}))+\frac{\mathrm{T}_{0} \mathrm{~L}}{\mathrm{EAR}} \mathrm{m}
$$

Collecting terms the deflection during loading becomes

$$
\theta=\frac{\mathrm{T}_{0}}{\mathrm{EA} \mu^{*}{ }_{\mathrm{eff}}}\left[\left(1+\mathrm{GF}^{*}\right) \mathrm{m}-\log (1+\mathrm{m})\right]
$$

Figure 3.2. shows the normalized deflection $\frac{\theta \mathrm{EA} \mu^{*} \text { eff }}{\mathrm{T}_{0}}$ as a function of the dimensionless moment $\mathrm{m}$ for several different GF. 
The open circuit drive, like the wrapped cable examined in Chapter 2, exhibits softening spring behavior, i.e. the stiffness decreases with increasing load. The drive's stiffness also decreases as the value of GF increases. To better understand these trends we solve for the drive's torsional stiffness $\mathrm{k}_{\mathrm{T}}$ by differentiating equation (3-17) with respect to $\mathrm{M}$ and rearranging terms to get

$$
\mathrm{k}_{\mathrm{T}}=\frac{\mathrm{dM}}{\mathrm{d} \theta}=\frac{\mathrm{dm}}{\mathrm{d} \theta} \frac{\mathrm{dM}}{\mathrm{dm}}=\frac{\mathrm{EA} \mu^{*}{ }_{\text {eff }} \mathrm{R}}{\left(\mathrm{GF}{ }^{*}+\frac{\mathrm{m}}{\mathrm{m}+1}\right)}
$$

Eliminating GF and $\mathrm{m}$ and rearranging some more we get

$$
\mathrm{k}_{\mathrm{T}}=\left[\left(\frac{\mathrm{EAR}{ }^{2}}{\mathrm{~L}}\right)^{-1}+\left(\mathrm{EA} \mu_{\text {eff }}^{*} \mathrm{R}\left(1+\frac{\mathrm{RT}_{0}}{\mathrm{M}}\right)\right)^{-1}\right]^{-1}
$$

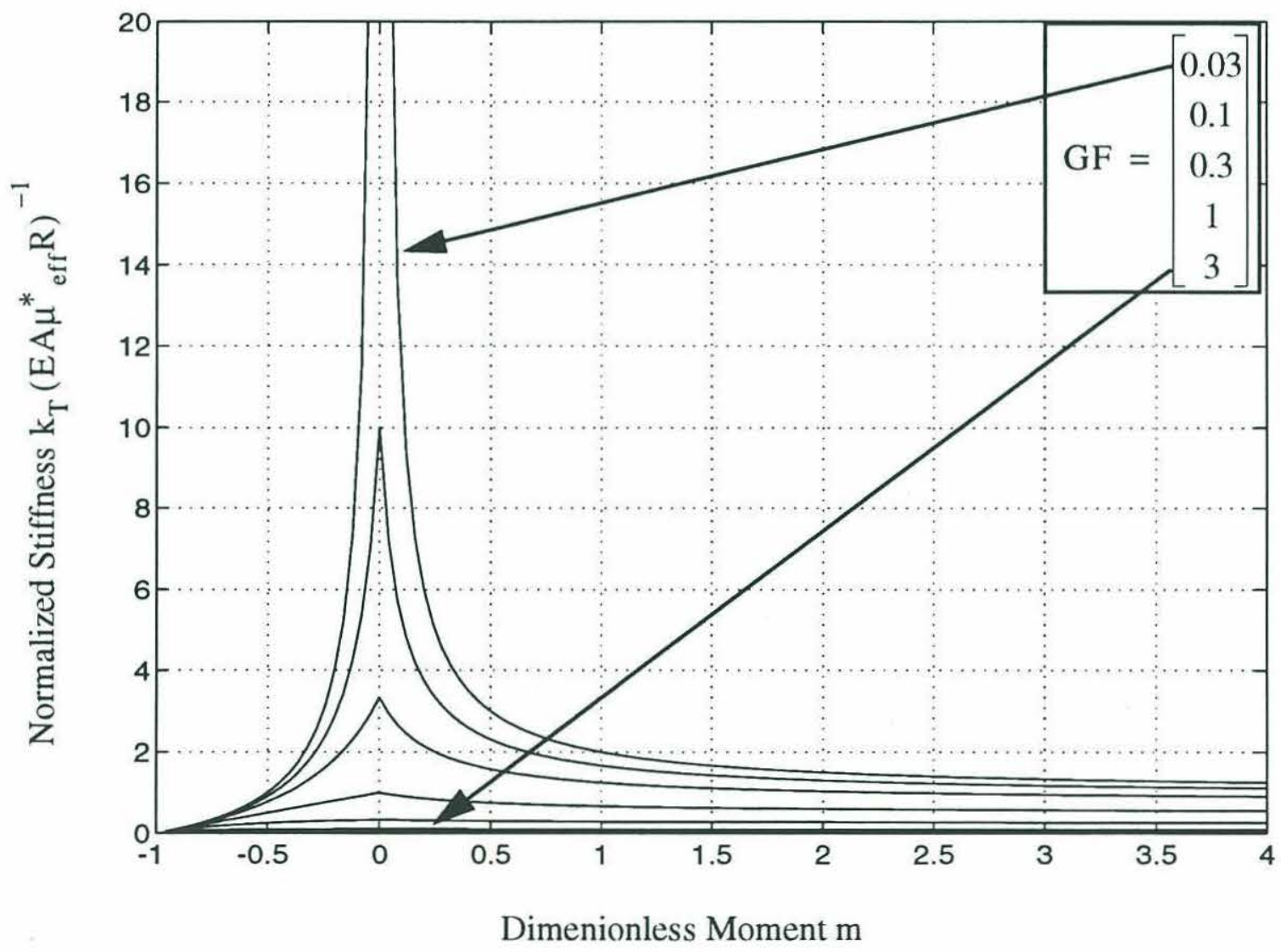

Figure 3.3. Stiffness of an Open Circuit Drive During Loading 
The drive's stiffness is the series combination of the stiffness due to the tangent cable (first term) and the stiffness due to the wrapped cable (second term). Note that $\mathrm{k}_{\mathrm{T}}$ depends on the pretension $\mathrm{T}_{0}$, i.e the higher the pretension the higher the stiffness. This indicates that we have some ability to change the stiffness of an existing drive without making any physical modifications to it. We shall see that this also holds true for closed circuit drives.

Figure 3.3. shows the normalized stiffness $\frac{\mathrm{k}_{\mathrm{T}}}{\mathrm{EA} \mu^{*}{ }_{\text {eff }} \mathrm{R}}$ as a function of the dimensionless moment $\mathrm{m}$ for several values of GF. The maximum stiffness for each curve $\mathrm{k}_{\mathrm{Tmax}}=\frac{\mathrm{EA} \mu_{\text {eff }} \mathrm{R}}{\mathrm{GF}}=\frac{\mathrm{EAR}^{2}}{\mathrm{~L}}$ occurs at $\mathrm{m}=0$ and is equal to the stiffness due to the tangent cable (the wrapped cable's stiffness is infinite at this point). As we apply a load the stiffness drops off rapidly, approaching zero as $\mathrm{m} \rightarrow-1$ (i.e. as the cable goes slack) or $\frac{\mathrm{EA} \mu_{\mathrm{eff}} \mathrm{R}}{\mathrm{GF}+1}$ as $\mathrm{m} \rightarrow+\infty$ (Note that when GF » 1 changes in $\mathrm{m}$ have little effect on the stiffness).

\subsubsection{Deflection During Unloading}

We find the pulley deflection $\theta$ during unloading by substituting the constitutive relation (3-10) and the dimensionless equilibrium constraint (3-3) into the geometric constraint (31) to get

$$
\theta=\frac{\mathrm{T}_{0}}{\mathrm{EA}^{*}{ }_{\text {eff }}}\left[2 \sqrt{\left(1+\mathrm{m}_{\mathrm{pk}}\right)(1+\mathrm{m})}-\mathrm{m}-2-\log \left(1+\mathrm{m}_{\mathrm{pk}}\right)\right]+\frac{\mathrm{LT}_{0}}{\mathrm{EAR}} \mathrm{m}
$$

Collecting terms the deflection during unloading becomes

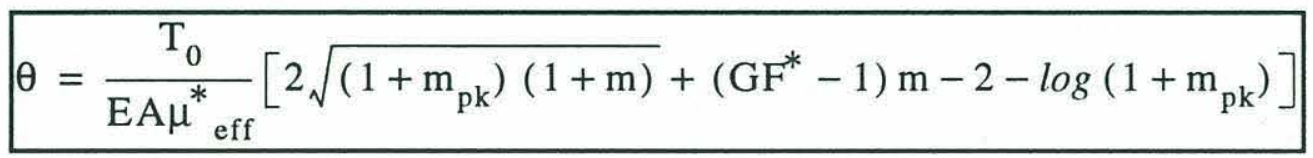

where $\mathrm{m}_{\mathrm{pk}}=\frac{\mathrm{M}_{\mathrm{pk}}}{\mathrm{RT}_{0}}$ is the peak dimensionless moment applied during loading.

Figure 3.4. shows the dimensionless moment $m$ vs. normalized deflection $\frac{\theta \mathrm{EA} \mu_{\text {eff }}^{*}}{\mathrm{~T}_{0}}$ of a drive having only wrapped cable (i.e. $\mathrm{GF}=0$ ). We show several possible unloading 
curves (dashed curves) branching off from the loading curve (solid curve), with each unloading curve corresponding to a different peak load $\mathrm{m}_{\mathrm{pk}}$ (curves to the right of the origin apply when $m_{p k}>0$ while curves to the left of the origin are unloading curves when $\mathrm{m}_{\mathrm{pk}}<0$ ). As an example, consider the drive when we change the dimensionless moment from zero to $m_{p k}=4$ and back again. During loading the deflection follows the loading curve up and to the right. As we begin to reduce the load the deflection immediately departs from the loading curve and proceeds downwards along the rightmost dashed curve. When $\mathrm{m}=0$ again the drive has not returned to its original position but instead remains deflected at about $\frac{1}{3}$ the maximum deflection value.

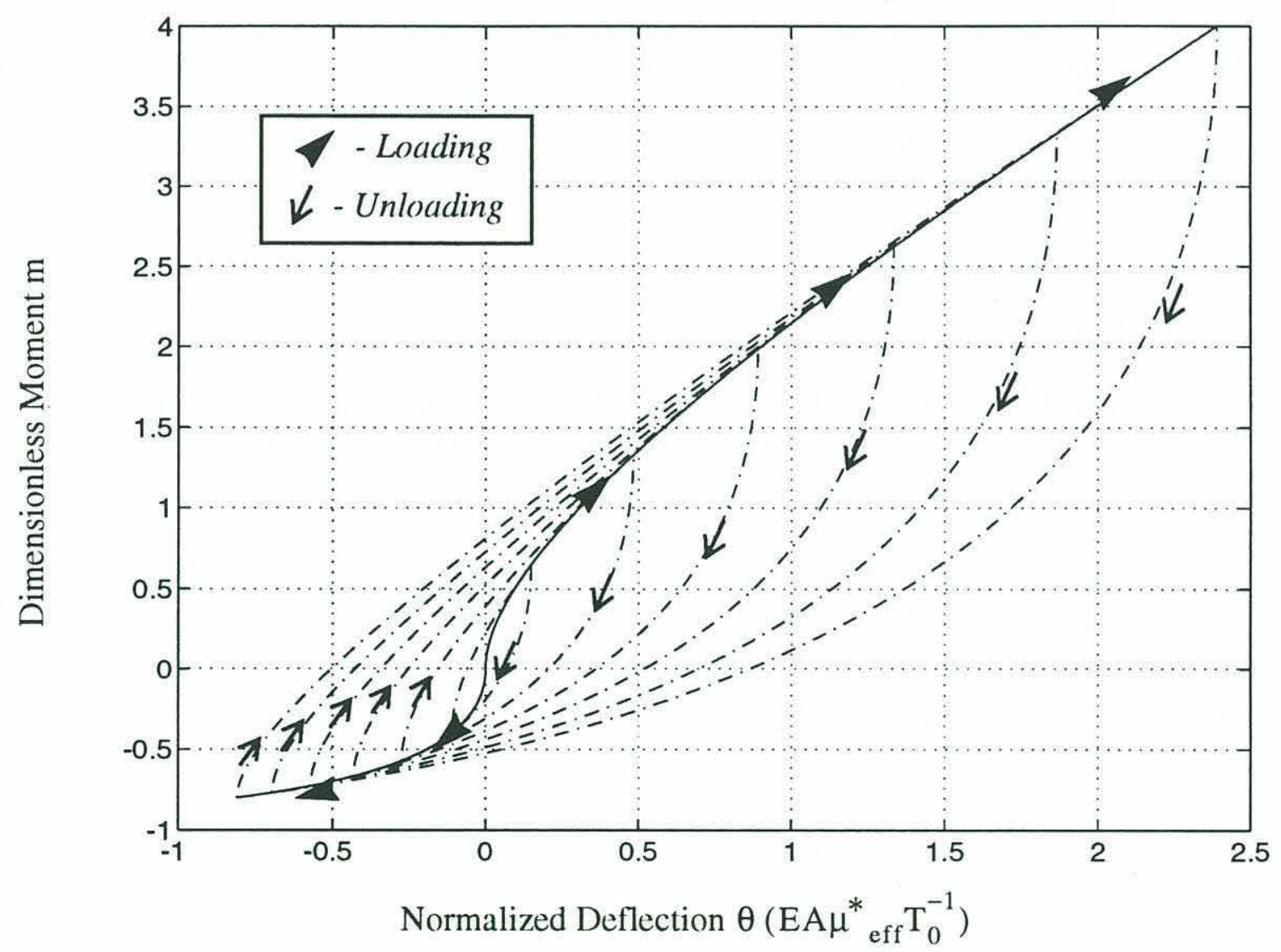

Figure 3.4. Deflection of an Open Circuit Cable Drive During Unloading, $G F=0$

If we continue to reduce the load such that $\mathrm{m}<0$ the unloading curve eventually intersects the loading curve again. When we load the drive the affected angle of wrap $\theta_{\text {aff }}$ forms, 
reaching its largest value when $m=m_{p k}$. As we remove this load the secondary affected angle of wrap $\alpha$ begins to form, progressively affecting more and more of the original region $\theta_{\text {aff }}$. If we reduce the load enough $\alpha$ eventually overtakes $\theta_{\text {aff }}$ at which point the original load $\mathrm{m}_{\mathrm{pk}}$ no longer affects the response of the drive. As we showed in section 2.2.5 this occurs when $\tau=\frac{1}{\tau_{\mathrm{pk}}}$, i.e. when

$$
\mathrm{m}=-\frac{\mathrm{m}_{\mathrm{pk}}}{1+\mathrm{m}_{\mathrm{pk}}}
$$

If we reduce the load beyond this point, the deflection once again follows the loading curve until we begin to reverse the load again. These results also apply when the initial loading is negative, i.e. when $\mathrm{m}_{\mathrm{pk}}<0$.

\subsubsection{Comments on the Validity of Open Circuit Models}

When developing these models the only section of cable we did not model exactly was the cable wrapped on the rotating pulley $\mathrm{R}$. To determine the exact elongation of this cable we would need to know the equilibrium tensile profile in the wrapped cable. However, because the pulley rotates during elongation the tensile profile depends (or may depend) on the cable elongation as a function of time. Solving this problem requires that we model the dynamics of the cable's response to the applied load.

For these reasons we chose to use the nonrotating pulley model to approximate the elongation of the rotating pulley system. We argued that any difference would be small if the pulley rotation $\theta$ was very small compared to the affected angle of wrap $\theta_{\text {aff }}$ expected for an equivalently loaded nonrotating pulley, i.e. if

$$
\theta \ll \phi=\frac{1}{\mu_{\mathrm{R}}^{*}} \log (1+\mathrm{m})
$$

We now consider how much smaller $\theta$ needs to be by estimating the error associated with this approximation. We concentrate on the elongation during loading, examining the two possible load cases: $\mathrm{M}<0$ and $\mathrm{M}>0$.

Applied Moment Negative $(\mathrm{M}<0)$ :

Referring to Figure 3.1., when $\mathrm{M}<0$ the pulley rotates in the negative $\theta$ direction which 
tends to reduce the affected angle of wrap by $\theta$. However, we know from the aggregate slip condition (2-19) that the affected angle of wrap must be at least $\theta_{\text {aff }}=\phi=\frac{1}{\mu_{R}} \log (\tau)$ for the cable to be in equilibrium. If $\theta_{\text {aff }}<\phi$ additional cable will slip until $\theta_{\text {aff }}=\phi$. Thus the affected angle of wrap at equilibrium always equals $\phi$, yielding the same tensile profile and elongation as the nonrotating pulley model.Thus the nonrotating model is exact when $\mathrm{M}<0$.

\section{Applied Moment Positive $(\mathrm{M}>0)$ :}

When $\mathrm{M}>0$ the pulley rotates in the positive $\theta$ direction, tending to increase the affected angle of wrap. Since the slip condition only requires that $\theta_{\text {aff }} \geq \phi$ it can no longer tell us the affected angle of wrap at equilibrium. However, we may conclude that the new affected angle of wrap is no larger than $\phi+\theta$ by considering the following limiting case. Assume that the usual exponential tensile profile propagates instantaneously out to $\theta_{\text {aff }}=\phi$ before the cable begins to deflect. The rotation of the pulley would then increase the affected angle of wrap to $\theta_{\text {aff }}=\phi+\theta$ (Note: This scenario is clearly impossible since there is no appreciable delay between the change in stress of a cable element and its change in elongation. However, this only means that the actual affected angle will always be less than $\phi+\theta$ ).

If we assume that $\theta \ll \phi$ we can estimate the percent error in elongation as

$$
\text { \%error }=\frac{\Delta \theta}{\theta} 100=\left(\frac{\left(\frac{\partial \theta}{\partial \theta_{\text {aff }}}\right) \Delta \theta_{\text {aff }}}{\theta}\right) 100
$$

Recognizing that the variation $\Delta \theta_{\text {aff }}=\theta$ we can write

$$
\frac{\Delta \theta}{\theta}=\frac{\partial \theta}{\partial \theta_{\text {aff }}}
$$

We recall that $\theta=\frac{\left(\delta_{R}+\delta_{L}+\delta_{r}\right)}{R}$ and note that the variation $\Delta \theta_{\text {aff }}$ only affects $\delta_{R}$. Therefore equation (3-25) becomes 


$$
\frac{\Delta \theta}{\theta}=\frac{\partial}{\partial \theta_{\text {aff }}}\left(\frac{\delta_{\mathrm{R}}}{\mathrm{R}}\right)
$$

Writing $\delta_{\mathrm{R}}$ as a function of $\theta_{\text {aff }}$ we get

$$
\delta_{\mathrm{R}}=\frac{\mathrm{T}_{0} \mathrm{R}}{\mathrm{EA} \mu_{\mathrm{R}}^{*}}\left(\mathrm{e}^{\mu^{*}{ }_{\mathrm{R}} \theta_{\text {aff }}}-1-\mu_{\mathrm{R}}^{*} \theta_{\mathrm{aff}}\right)
$$

which leads to

$$
\frac{\Delta \theta}{\theta}=\frac{\mathrm{T}_{0}}{\mathrm{EA}}\left(\mathrm{e}^{\mu^{*}{ }_{\mathrm{R}} \theta_{\text {aff }}}-1\right)
$$

Substituting the nominal value of $\theta_{\text {aff }}$, i.e.

$$
\theta_{\mathrm{aff}}=\phi=\frac{1}{\mu_{\mathrm{R}}^{*}} \log (1+\mathrm{m})
$$

the percent error in the predicted deflection when $\theta \ll \theta_{\text {aff }}$ becomes

$$
\text { \%error }=\frac{\Delta \theta}{\theta} 100=\left(\frac{\mathrm{T}_{0}}{\mathrm{EA}} \mathrm{m}\right) 100
$$

For real drives, $m$ will typically range from -1 to 4 and is unlikely to ever exceed 5. Fatigue life considerations usually limit $\frac{\mathrm{T}_{0}}{\mathrm{EA}}$ (the initial strain in the cable due to the preload) to be 0.0025 at most. Thus our worst case error in predicted deflection due to the increase in wrap angle would be $1.3 \%$. As a final step we verify our original assumption that $\theta \ll \phi$ by substituting these values for $\mathrm{m}$ and $\frac{\mathrm{T}_{\mathrm{o}}}{\mathrm{EA}}$ into

$$
\frac{\theta}{\phi}=\frac{\mathrm{T}_{0}}{\mathrm{EA}}\left[\left(1+\mathrm{GF}^{*}\right) \frac{\mathrm{m}}{\log (1+\mathrm{m})}-1\right]
$$

For $\mathrm{m}=5$ and $\frac{\mathrm{T}_{0}}{\mathrm{EA}}=0.0025$ this ratio will he less than 0.05 for $\mathrm{GF}$ values less than 6.5 . 
For higher GF values our assumption that $\theta$ « $\phi$ eventually breaks down which means that equation (3-30) will no longer hold. Qualitatively, we expect the modelling error for the cable wrapped on the rotating pulley to grow as we increase GF. However we expect that the significance of this error will decrease since large GF values indicate that wrapped cable plays a small role in the deflection of the drive. Thus we conclude that the nonrotating model can be used to accurately model the elongation of the cable wrapped on the rotating pulley.

\subsection{Experimental Confirmation}

In this section we show that the deflection models agree closely with data obtained from experiments involving real open circuit drives. We present the theoretical basis for the experiment and follow with a presentation and discussion of the results. For a description of the experimental apparatus and procedures used see Appendix A.

\subsubsection{Approach}

By definition our models only apply once the drive reaches its equilibrium deflection. This gives us two options for collecting data during a trial; apply an "increment/wait/measure" strategy or increase the load quasi-statically and measure the deflection continuously. We choose the latter approach because it enables us to collect data more efficiently.

From each trial we obtain a vector of applied loads $\stackrel{M}{ }$ and an associated vector of deflections $\theta$ (the " $\bullet "$ to signifies that the quantity is a sequence values). If the loading model (316) is correct the relationship between these vectors will be

$$
\theta=\frac{\mathrm{T}_{0}}{\mathrm{EA}^{*}{ }_{\text {eff }}}\left(\frac{\stackrel{\circ}{\mathrm{RT}_{0}}}{\log }\left(1+\frac{\stackrel{\circ}{\mathrm{RT}_{0}}}{\mathrm{EAR}^{2}}\right)-\frac{\mathrm{L}}{\mathrm{M}}\right.
$$

Defining the deflection of the wrapped cable $\hat{\theta}_{\mathrm{wr}}$ and the processed load vector $\ell_{1}$ as

$$
\begin{aligned}
& \hat{\theta}_{\mathrm{wr}} \equiv \hat{\theta}-\frac{\mathrm{L}}{\mathrm{EAR}^{2}} \stackrel{\mathrm{M}}{\mathrm{H}}
\end{aligned}
$$

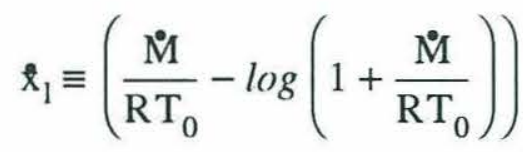


equation (3-32) becomes

$$
\hat{\theta}_{\mathrm{wr}}=\frac{\mathrm{T}_{0}}{\mathrm{EA} \mu_{\text {eff }}^{*}} \mathrm{\ell}_{1}
$$

For the values of $\hat{\theta}_{\mathrm{wr}}$ associated with the unloading portion of a trial we employ a similar strategy. Equation (3-20) gives the deflection during unloading which we can rewrite as

$$
\theta_{\mathrm{wr}}=\frac{\mathrm{T}_{0}}{\mathrm{EA} \mu_{\text {eff }}^{*}} \mathrm{x}_{\mathrm{u}}
$$

where we define the processed load vector during unloading $\hat{x}_{\mathrm{u}}$ as

$$
\mathrm{t}_{\mathrm{u}}=2 \sqrt{\left(1+\frac{\mathrm{M}_{\mathrm{pk}}}{\mathrm{RT}_{0}}\right)\left(1+\frac{\stackrel{\circ}{\mathrm{RT}_{0}}}{\mathrm{RT}_{0}}\right)}-\frac{\stackrel{\circ}{\mathrm{R}}}{\mathrm{R}_{0}}-\log \left(1+\frac{\mathrm{M}_{\mathrm{pk}}}{\mathrm{RT}_{0}}\right)
$$

Thus we check the validity of the models by determining the linearity of the relationship between $\theta_{\mathrm{wr}}$ and the appropriate processed load vector. In addition we can determine the coefficient of friction $\mu^{*}$ eff from slopes of these lines. (Note also that both lines should be parallel and, because they share a common point (the peak deflection) they should also be coincident). Unfortunately, recalling that $\mu^{*}{ }_{\text {eff }}=\left(\frac{r}{R} \frac{1}{\mu_{r}}+\frac{1}{\mu_{R}}\right)^{-1} \operatorname{sgn}(\mathrm{M})$, we see that we cannot directly determine the coefficient of friction for given pulley in a dual pulley drive. If we set $r=0$, however, this becomes $\mu^{*}{ }_{\text {eff }}=\mu_{R} s g n(M)$, allowing us to solve directly for the coefficient of friction on pulley $R$ (Physically, setting $r=0$ means that the only wrapped cable in the drive is on pulley $R$ ). Thus we test both dual pulley (i.e. $r \neq 0$ ) and single pulley $(\mathrm{r}=0)$ open circuit drives.

In summary, our analysis of the data consists of two tasks.

1. prove the validity of the models by showing that $\hat{\theta}_{\mathrm{wr}}$ and the processed load vectors $\mathrm{t}_{1}$ and $\mathrm{x}_{\mathrm{u}}$ are linearly related

2. determine and compare the coefficients of friction obtained from the various trials 


\subsubsection{A Typical Trial}

Figure 3.5. shows the wrap deflection $\theta_{\mathrm{wr}}$ and the dimensionless moment $\mathrm{m}$ as functions of time for a typical experimental trial. We note immediately that our assumption of quasistatic loading is incorrect because the deflection continues to change after we stop changing the applied load. Strictly speaking, then, our models only apply at the two equilibrium points (A) and (B). However, we show below that the models still fit the rest of the data extremely well, indicating that the loading is very nearly quasi-static. Therefore we still use the deflection/processed load vector plots to show the validity of the models over a range of load values. However the slopes of these plots no longer gives the correct value of the coefficient of friction but instead represents some combination of Coulomb and viscous friction forces which we call the acting coefficient of friction. We determine the true coefficient of friction by applying equations (3-35) to and (3-36) to the two equilibrium points (A) and (B), respectively.
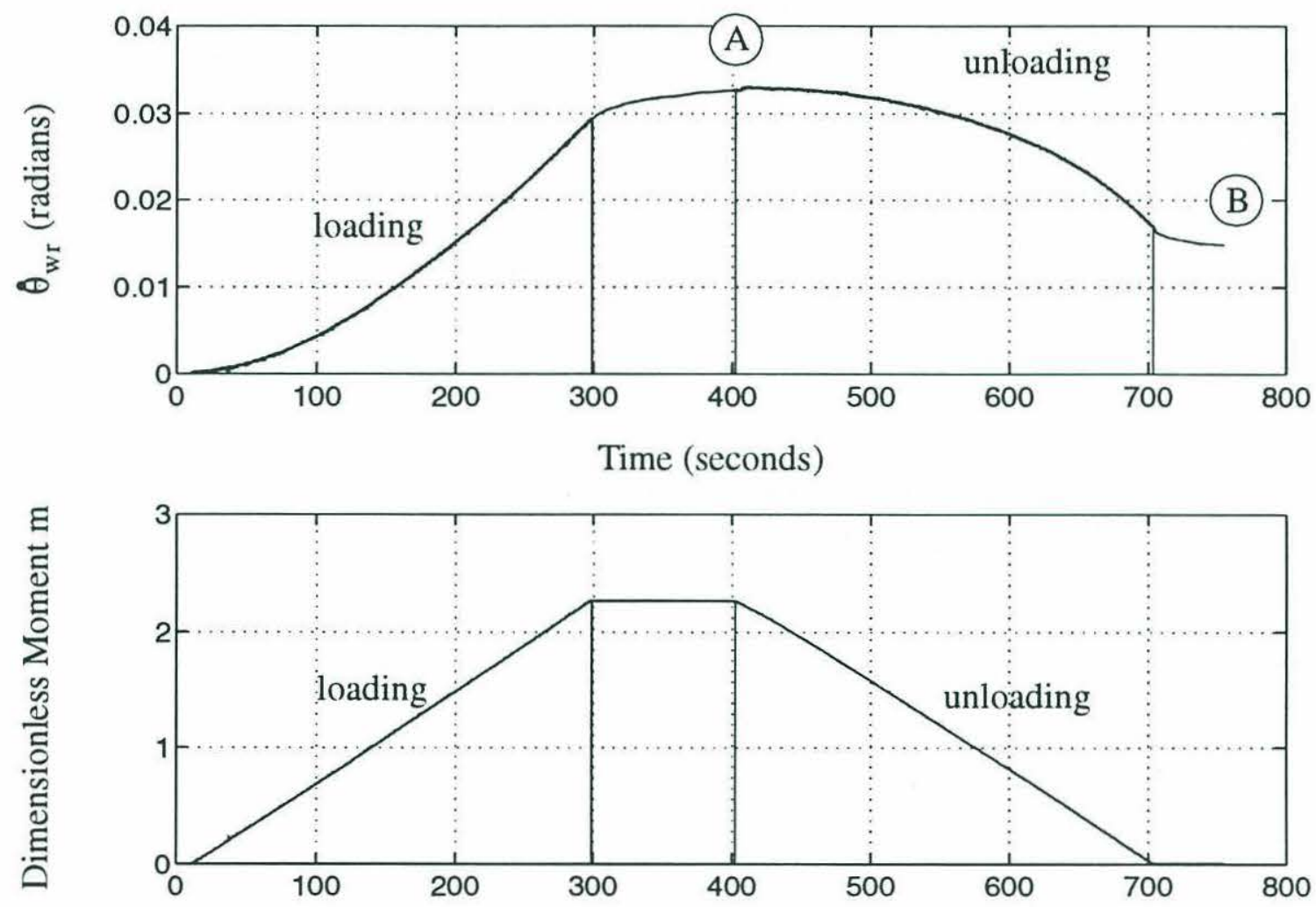

Time (seconds)

Figure 3.5. Wrap Deflection $\hat{\theta}_{\mathrm{wr}}$ and Dimensionless Moment $\mathrm{m}$ vs. Time, Trial 145 
Considering how slowly we change the applied load (0.028 to 0.098 in-lbf per second) the fact that the loading is not quasi-static implies the presence of very substantial viscous forces between the cable and the pulley. If true, the drive's dynamic response may depend strongly on load rate, exhibiting greater stiffness for rapid changes in load and lower stiffness for more gradual changes.

\section{Unprocessed Data}

Figure 3.6. shows the dimensionless applied moment $\mathrm{m}$ and wrapped cable deflection $\hat{\theta}_{\mathrm{wr}}$ from Figure 3.5. plotted against each other. The two horizontal portions of the curve represent the deflection that takes place between the time when we stop changing the applied load and the time when the drive reaches equilibrium. Note the similarity of the loading and unloading curves to those shown in Figure 3.4.

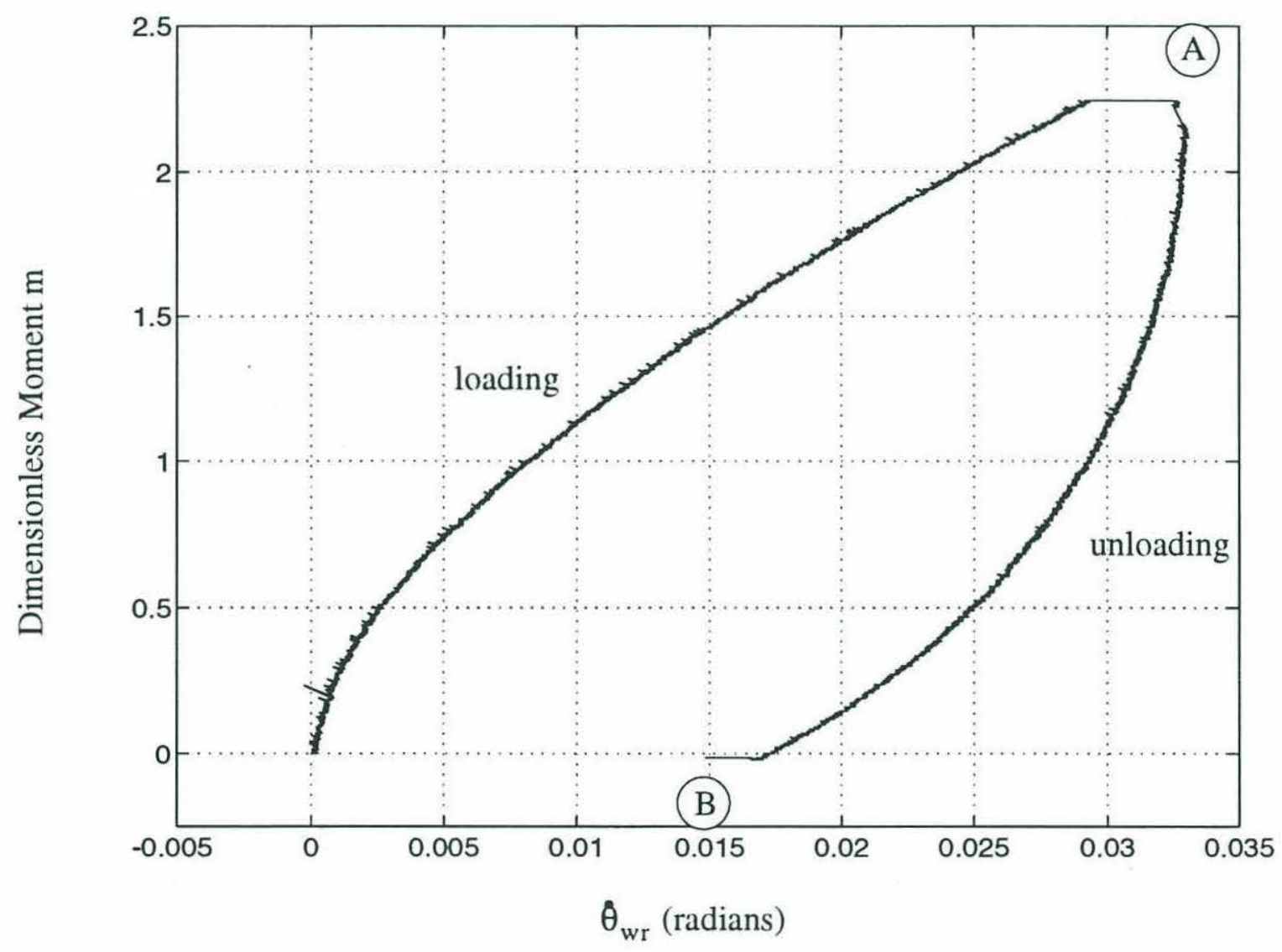

Figure 3.6. Dimensionless moment vs. Normalized Deflection, Trial 145 


\section{Processed Data}

Figure 3.7. shows that each of the processed load vectors $k_{1}$ and $k_{u}$ varies linearly with $\theta_{\mathrm{wr}}$ as predicted by the loading and unloading models. To quantify the agreement we perform a least squares fit of a straight line to the data and determine the associated correlation coefficient for each curve. For this trial the acting coefficients of friction (obtained from the slopes) during loading and unloading are 0.056 and 0.605 and the associated correlation coefficients are both 0.9998 . The true coefficients of friction (obtained from the equilibrium points) are $0.048 \pm 0.008$ and $0.043 \pm 0.008$.

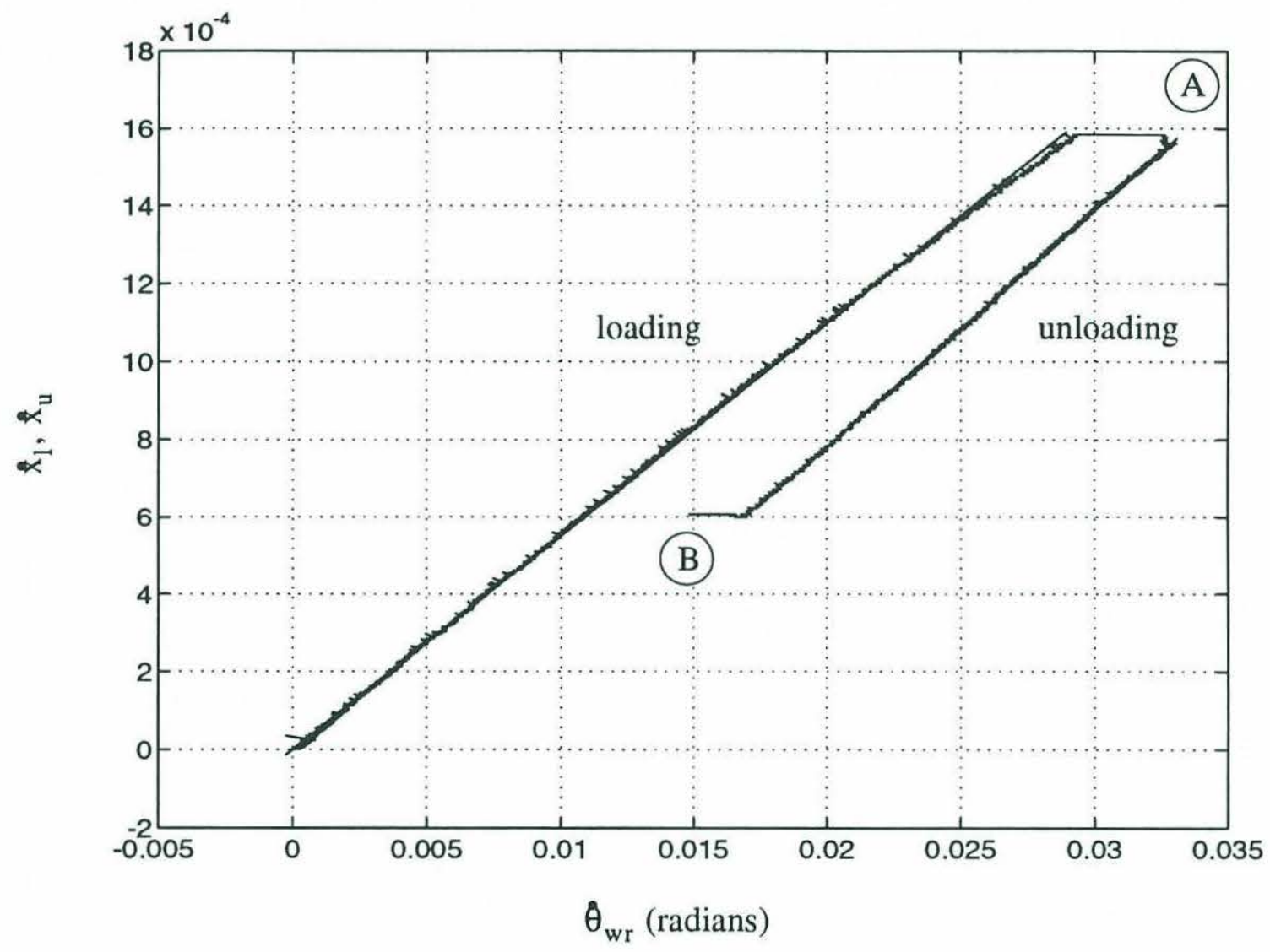

Figure 3.7. $\mathrm{x}_{1}$ and $\mathrm{k}_{\mathrm{u}}$ vs. $\hat{\theta}_{\mathrm{wr}}$, Trial 145

\subsubsection{Correlation Coefficients: All Trials}

The correlation coefficient indicates how well the model fits the data. Figure 3.8. shows the correlation coefficients associated with the loading (circles) and unloading (x's) models for each of the open circuit trials. This plot indicates that both models fit the data very closely for each trial. The groups of trials that dip noticeably from the rest are trials in which we 
applied and then removed a negative moment (i.e. $\mathrm{M}_{\mathrm{pk}}<0$ ).

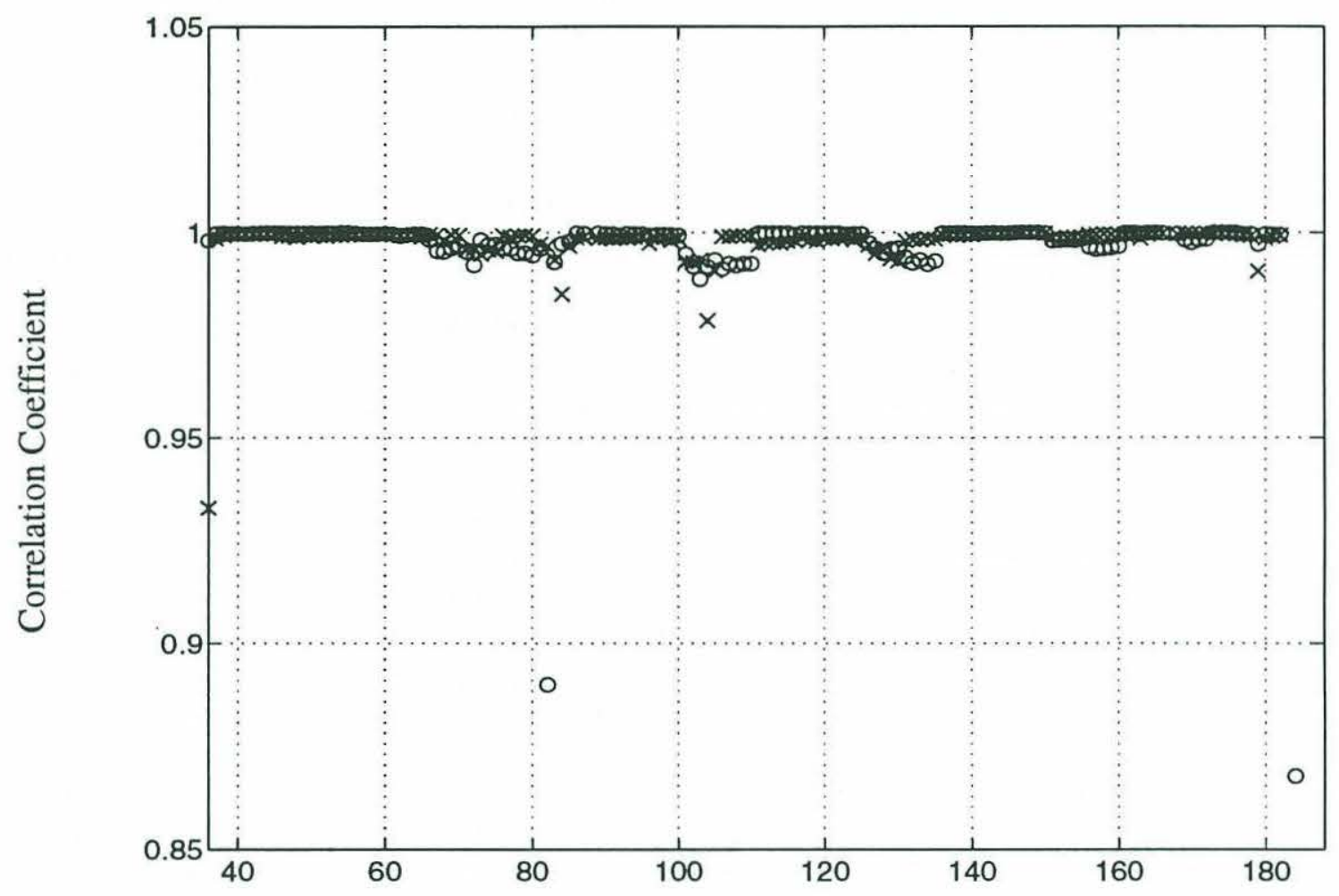

Trial Number

Figure 3.8. Correlation Coefficients, Trials 36 through 188

\subsubsection{Coefficient of Friction: All Trials}

\section{True Coefficient of Friction:}

Referring to Table 3.1 we see that the coefficients of friction obtained from the loading and unloading models are in very close agreement for most of the trial groups. However if we look at the values obtained from a given trial (see any of Figure C.1. through Figure C.5 in Appendix B) we see that the $\mu_{t}$ 's obtained from the loading portion of the trials (circles) are almost always higher than those from the unloading portion of trials (crosses) when the peak load $M_{p k}$ is positive. Since this situation reverses when $M_{p k}$ is negative we believe it is related to a directionally dependent effect that we have not adequately compensated for, probably bearing friction (despite our attempts to characterize and compensate for it). 


\begin{tabular}{|c|c|c|c|c|c|c|}
\hline \multirow[b]{2}{*}{ Trials } & \multirow[b]{2}{*}{$\mathrm{R}$ (in) } & \multirow[b]{2}{*}{ r (in) } & \multicolumn{2}{|c|}{$\begin{array}{c}\text { True Coefficient of } \\
\text { Friction }\end{array}$} & \multicolumn{2}{|c|}{$\begin{array}{l}\text { Acting Coefficient of } \\
\text { Friction }\end{array}$} \\
\hline & & & $\begin{array}{l}\text { Loading } \\
\text { Mean } \\
\text { (St. Dev.) } \\
\text { [Uncert.] }\end{array}$ & $\begin{array}{l}\text { Unloading } \\
\text { Mean } \\
\text { (St. Dev.) } \\
\text { [Uncert.] }\end{array}$ & $\begin{array}{l}\frac{\text { Loading }}{\text { Mean }} \\
\text { (St. Dev.) } \\
\text { [Uncert.] }\end{array}$ & $\begin{array}{l}\text { Unloading } \\
\text { Mean } \\
\text { (St. Dev.) } \\
\text { [Uncert.] }\end{array}$ \\
\hline $36-65$ & $\begin{array}{c}2.438^{\prime \prime} \\
\text { Aluminum }\end{array}$ & 0 & $\begin{array}{c}0.12 \\
(0.005) \\
{[0.008]}\end{array}$ & $\begin{array}{c}0.12 \\
(0.015) \\
{[0.03]}\end{array}$ & $\begin{array}{c}0.13 \\
(0.006) \\
{[-]}\end{array}$ & $\begin{array}{c}0.15 \\
(0.005) \\
{[-]}\end{array}$ \\
\hline $86-100$ & $\begin{array}{c}1.438 " \\
\text { Aluminum }\end{array}$ & 0 & $\begin{array}{c}0.092 \\
(0.010) \\
{[0.008]}\end{array}$ & $\begin{array}{c}0.084 \\
(0.011) \\
{[0.02]}\end{array}$ & $\begin{array}{c}0.10 \\
(0.010) \\
{[-]}\end{array}$ & $\begin{array}{c}0.11 \\
(0.011) \\
{[-]}\end{array}$ \\
\hline $111-125$ & $\begin{array}{c}0.688 " \\
\text { Aluminum }\end{array}$ & 0 & $\begin{array}{c}0.062 \\
(0.008) \\
{[0.01]}\end{array}$ & $\begin{array}{c}0.058 \\
(0.009) \\
{[0.01]}\end{array}$ & $\begin{array}{c}0.068 \\
(0.008) \\
{[-]}\end{array}$ & $\begin{array}{c}0.078 \\
(0.007) \\
{[-]}\end{array}$ \\
\hline $136-150$ & $\begin{array}{c}1.438 " \\
\text { Aluminum }\end{array}$ & $\begin{array}{c}2.438 " \\
\text { Aluminum }\end{array}$ & $\begin{array}{c}0.047 \\
(0.001) \\
{[0.003]}\end{array}$ & $\begin{array}{r}0.043 \\
(0.002) \\
{[0.006]}\end{array}$ & $\begin{array}{c}0.054 \\
(0.002) \\
{[-]}\end{array}$ & $\begin{array}{c}0.060 \\
(0.002) \\
{[-]}\end{array}$ \\
\hline $161-168$ & $\begin{array}{l}1.438 " \\
\text { Steel }\end{array}$ & $\begin{array}{c}2.438 " \\
\text { Aluminum }\end{array}$ & $\begin{array}{c}0.044 \\
(0.002) \\
{[0.002]}\end{array}$ & $\begin{array}{c}0.043 \\
(0.005) \\
{[0.004]}\end{array}$ & $\begin{array}{c}0.049 \\
(0.002) \\
{[-]}\end{array}$ & $\begin{array}{c}0.053 \\
(0.003) \\
{[-]}\end{array}$ \\
\hline $173-176$ & $\begin{array}{l}1.438 " \\
\text { Steel }\end{array}$ & $\begin{array}{c}1.438 " \\
\text { Aluminum }\end{array}$ & $\begin{array}{c}0.058 \\
(0.001) \\
{[0.003]}\end{array}$ & $\begin{array}{l}0.054) \\
(0.001 \\
{[0.01]}\end{array}$ & $\begin{array}{c}0.064 \\
(0.001) \\
{[-]}\end{array}$ & $\begin{array}{c}0.073 \\
(0.001) \\
{[-]}\end{array}$ \\
\hline $177-182$ & $\begin{array}{l}2.438 " \\
\text { Steel }\end{array}$ & $\begin{array}{c}2.438^{\prime \prime} \\
\text { Aluminum }\end{array}$ & $\begin{array}{c}0.083 \\
(0.006) \\
{[0.006]}\end{array}$ & $\begin{array}{c}0.083 \\
(0.012) \\
{[0.007]}\end{array}$ & $\begin{array}{c}0.085 \\
(0.006) \\
{[-]}\end{array}$ & $\begin{array}{c}0.088 \\
(0.003) \\
{[-]}\end{array}$ \\
\hline
\end{tabular}

Table 3.1 Mean Values of True and Acting Coefficients of Friction 


\section{Acting Coefficient of Friction:}

Referring again to Table 3.1 we see that the average coefficients of friction obtained from the loading and unloading portions of the trials are again in good agreement, though they are not as close as the true coefficients of friction are. Moving to Appendix B we see that the shape of the $\mu_{\mathrm{a}}$ curves closely track the shape of the $\mu_{\mathrm{t}}$ curves, further indicating that the loading is in fact fairly close to being quasi-static (note, however, that now the $\mu_{\mathrm{a}}$ 's from the loading portion of the trials (circles) ar consistently lower than those from the unloading portions of the trials (crosses) when $\mathrm{M}_{\mathrm{pk}}>0$ and vice versa when $\mathrm{M}_{\mathrm{pk}}<0$. We have no explanation for this.)

\subsection{Conclusions}

Using the results of Chapter 2 we modelled the deflection of a single stage open circuit cable drive during loading (equation (3-17)) and unloading (equation (3-21)). Our experiments demonstrate the validity of these models but the inherent variability of the coefficient of friction limits the accuracy with which we can predict the response of a particular drive to, say, $\mp 20$ percent. From these models we determined the key characteristics of open circuit cable drives:

\section{Characteristics of Open Circuit Cable Drives}

\section{Visco-elastic Deflection Response}

The drives we tested required a significant amount of time to reach their equilibrium deflections. We believe viscous friction forces between the cable and pulley to be responsible for this behavior. If true, the stiffness of cable drives should be higher when the applied load varies rapidly.

\section{Softening Spring behavior}

Cable drives are stiffest near zero load, becoming progressively more compliant as the load increases.

3. Nature of Response Determined Value of $\mathrm{GF}=\frac{\mathrm{L} \mu_{\mathrm{eff}}}{\mathrm{R}}$.

The value of the geometry-friction number GF indicates the relative importance of the linear and nonlinear (softening) components of the drive's stiffness. A high GF value yields a drive with very low but nearly linear stiffness. A low GF value results in a much stiffer drive but this stiffness drops off rapidly as the load increases. Most reasonably compact drives will have a low value of GF which means they will exhibit significant softening spring behavior. 
4. Stiffness Depends on Pretension $\mathrm{T}_{0}$.

Increasing $\mathrm{T}_{0}$ makes a cable drive stiffer. The effect of a given change in pretension depends on the value of GF.

5. Inherent hysteresis

Hysteresis is inherent to cable drives. However, it is predictable 


\section{Chapter 4 Closed Circuit Cable/Pulley Drives}

In this chapter we determine the equilibrium deflection of a single stage closed circuit cable drive by modelling it as two opposing open circuit drives in parallel. As with the open circuit drive the geometry friction number GF determines the character of a closed circuit drive's deflection during loading and unloading. In general we cannot find explicit solutions for the equilibrium cable tensions but there are two special cases in which we can; when $\mathrm{GF}=\infty$ (i.e. pulleys widely separated) and when GF $=0$ (pulleys nearly tangent). By comparison with numerical solutions for other GF values we show that most cable drives can be accurately modelled by using the one of the special case solutions to approximate their equilibrium tensions. We end by presenting experimental data which confirms the validity of the GF $=0$ based approximation for a particular drive. 


\subsection{The Closed Circuit Cable Drive}

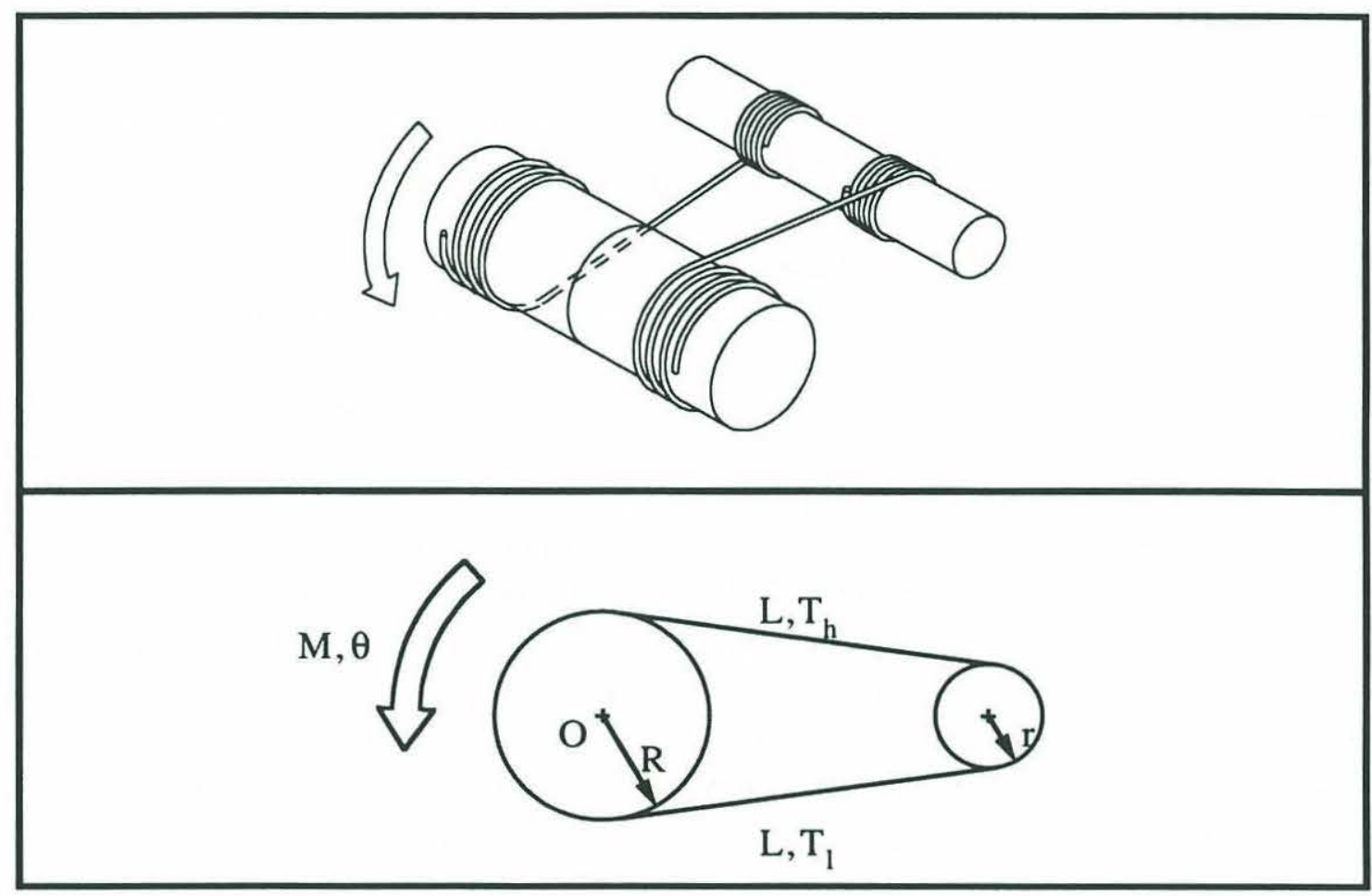

Figure 4.1. Closed Circuit Cable Drive.

Definition: $A$ closed circuit cable drive is a cable drive whose cables and pulleys form a closed force path such that the cables are in a state of tension regardless even when the drive transmits no load.

Figure 4.1. shows a single stage pretensioned closed circuit cable drive. We can think of the closed circuit drive as two opposing open circuit drives which link the same two shafts. We pretension the drive by applying opposing torques to the two halves of pulley $\mathrm{R}$ and then clamping the halves together. When we subsequently remove the torques the cables try to return to their unstressed length but cannot because the two halves of the pulley can no longer turn relative to one another. Thus the drive remains in a state of tension. We uniformly pretension the drive by, prior to clamping, rotating the loaded drive through its full range of motion. This ensures that the cable tension is everywhere equal to some value $\mathrm{T}_{0}$.

Having uniformly pretensioned the drive and having fixed the position of pulley $r$, we wish to determine the equilibrium deflection of pulley $\mathrm{R}$ when we apply and subsequently remove a moment $\mathrm{M}$. 


\subsection{Modelling}

As with the open circuit drive, we find the deflection by identifying and then simultaneously satisfying the system's geometric, constitutive and equilibrium constraints. In these derivations we assume that

1. the affected angles of wrap are always smaller than actual angles of wrap.

2. deflection angle is much smaller than the slip angles.

3. the applied moment is positive.

4. the lower cable does not go slack.

\section{Geometric Constraints:}

When pulley $R$ deflects by a positive amount $\theta$ the length of the upper cable increases by $\theta \mathrm{R}$ while the length of the lower cable decreases by this same amount. The geometric constraint is therefore

$$
\Sigma \text { Elongations }=0=\delta_{\mathrm{h}}+\delta_{1}
$$

where $\delta_{\mathrm{h}}$ and $\delta_{1}$ are the changes in length of the upper and lower cables. In addition, the rotation $\theta$ of the output pulley equals the cable elongation $\delta_{\mathrm{h}}$ divided by the pulley radius $\mathrm{R}$ or

$$
\theta=\frac{\delta_{\mathrm{h}}}{\mathrm{R}}=-\frac{\delta_{1}}{\mathrm{R}}
$$

\section{Equilibrium Constraint:}

At equilibrium the moments acting on pulley $\mathrm{R}$ must sum to zero which requires that

$$
\Sigma \mathrm{M}_{\mathrm{o}}=0=\mathrm{M}-\left(\mathrm{T}_{\mathrm{h}}-\mathrm{T}_{1}\right) \mathrm{R}
$$

Dividing by $\mathrm{T}_{0}$ we obtain the dimensionless equilibrium constraint

$$
\mathrm{m}=\tau_{\mathrm{h}}-\tau_{1}
$$

where we define the dimensionless tensions $\tau_{\mathrm{h}} \equiv \frac{\mathrm{T}_{\mathrm{h}}}{\mathrm{T}_{0}}$ and $\tau_{1} \equiv \frac{\mathrm{T}_{1}}{\mathrm{~T}_{0}}$ and the dimensionless 
moment $\mathrm{m} \equiv \frac{\mathrm{M}}{\mathrm{RT}_{0}}$.

\section{Constitutive Relations:}

In Chapter 3 we showed that during loading the constitutive relation for a cable wrapped around two pulleys is given by equation (3-9). When we apply a positive moment $M$ the tension in the upper cable increases from $T_{0}$ to $T_{h}$ while the tension in the lower cable decreases from $T_{0}$ to $T_{1}$ meaning that $\tau_{h}>1$ and $\tau_{1}<1$. Applying equations (3-9) and (38 ) we find the constitutive relations during loading for the upper and lower cable to be

$$
\begin{aligned}
& \delta_{\mathrm{h}}=\frac{\mathrm{RT}_{0}}{\mathrm{EA} \mu_{\text {heff }}}\left(\tau_{\mathrm{h}}-1-\log \tau_{\mathrm{h}}\right)+\frac{\mathrm{LT}_{0}}{\mathrm{EA}}\left(\tau_{\mathrm{h}}-1\right) \\
& \delta_{1}=-\frac{\mathrm{RT}_{0}}{\mathrm{EA} \mu_{\text {leff }}}\left(\tau_{1}-1-\log \tau_{1}\right)+\frac{\mathrm{LT}_{0}}{\mathrm{EA}}\left(\tau_{1}-1\right)
\end{aligned}
$$

where

$$
\begin{aligned}
& \frac{1}{\mu_{\mathrm{heff}}}=\left(\frac{\mathrm{r}}{\mathrm{R}} \frac{1}{\mu_{\mathrm{hr}}}+\frac{1}{\mu_{\mathrm{hR}}}\right) \\
& \frac{1}{\mu_{\mathrm{leff}}}=\left(\frac{\mathrm{r}}{\mathrm{R}} \frac{1}{\mu_{\mathrm{lr}}}+\frac{1}{\mu_{\mathrm{lR}}}\right)
\end{aligned}
$$

(Note: We use $\mu$ instead of $\mu^{*}$ because we assume that $M$ is always positive. Because of its symmetry the closed circuit drive (in contrast to the open circuit) responds the same. to positive and negative loads. Note also that, for the time being, we allow for a different coefficient of friction at each cable/pulley interface).

Similarly, we apply equation (3-10) to find that the constitutive relations during unloading are

$$
\begin{aligned}
& \delta_{\mathrm{h}}=\frac{\mathrm{RT} \mathrm{T}_{0}}{\mathrm{EA} \mu_{\mathrm{heff}}}\left(2 \sqrt{\tau_{\mathrm{h}_{\mathrm{pk}}} \tau_{\mathrm{h}}}-\tau_{\mathrm{h}}-1-\log \tau_{\mathrm{h}}\right)+\frac{\mathrm{T}_{0} \mathrm{~L}}{\mathrm{EA}}\left(\tau_{\mathrm{h}}-1\right) \\
& \delta_{1}=-\frac{\mathrm{RT}_{0}}{\mathrm{EA} \mu_{\text {leff }}}\left(2 \sqrt{\tau_{\mathrm{l}_{\mathrm{pk}}} \tau_{1}}-\tau_{1}-1-\log \tau_{\mathrm{l}_{\mathrm{pk}}}\right)+\frac{\mathrm{T}_{0} \mathrm{~L}}{\mathrm{EA}}\left(\tau_{1}-1\right)
\end{aligned}
$$


where $\tau_{\mathrm{h}_{\mathrm{pk}}}$ and $\tau_{\mathrm{l}_{\mathrm{pk}}}$ are the upper and lower cable tensions associated with $\mathrm{M}_{\mathrm{pk}}$, the peak moment applied during the loading phase.

\subsubsection{Equilibrium Equations During Loading}

\section{Equilibrium Tensions:}

To find the equilibrium tensions during loading we substitute the constitutive relations (45) and (4-6) into the geometric constraint (4-1) to get

$$
\frac{\mathrm{RT}_{0}}{\mathrm{EA} \mu_{\text {heff }}}\left(\tau_{\mathrm{h}}-1-\log \tau_{\mathrm{h}}\right)-\frac{\mathrm{RT}_{0}}{\mathrm{EA} \mu_{\text {leff }}}\left(\tau_{1}-1-\log \tau_{1}\right)+\frac{\mathrm{LT}_{0}}{\mathrm{EA}}\left(\tau_{1}+\tau_{\mathrm{h}}-2\right)=0
$$

Dividing through by $\frac{\mathrm{RT}_{0}}{\mathrm{EA} \mu_{\text {heff }}}$ and rearranging yields

$$
\frac{\mu_{\text {heff }}}{\mu_{\text {leff }}} \log \tau_{1}-\log \tau_{h}+\tau_{h}-\frac{\mu_{\text {heff }}}{\mu_{\text {leff }}} \tau_{1}+\frac{\mu_{\text {heff }}}{\mu_{\text {leff }}}-1+\frac{L \mu_{\text {heff }}}{R}\left(\tau_{1}+\tau_{h}-2\right)=0
$$

We now use the dimensionless equilibrium constraint (4-4) to eliminate $\tau_{\mathrm{h}}$ and rearrange some more to get

$$
\frac{\mu_{\text {heff }}}{\mu_{\text {leff }}} \log \tau_{1}-\log \left(\tau_{1}+m\right)+\left(1-\frac{\mu_{\text {heff }}}{\mu_{\text {leff }}}\right)\left(\tau_{1}-1\right)+m+\frac{L \mu_{\text {heff }}}{R}\left(2 \tau_{1}+m-2\right)=0
$$

Recognizing $\frac{\mathrm{L} \mu_{\mathrm{heff}}}{\mathrm{R}}$ as a form of the geometry friction number we can rewrite this as

$$
\log \left(\frac{\tau_{1}^{\frac{\mu_{\text {heff }}}{\mu_{\text {leff }}}}}{\tau_{1}+\mathrm{m}}\right)+(1+\mathrm{GF}) \mathrm{m}+\left(1-\frac{\mu_{\text {heff }}}{\mu_{\text {leff }}}+2 \mathrm{GF}\right)\left(\tau_{1}-1\right)=0
$$

At this point we make the simplifying assumption that $\mu_{\text {heff }} \approx \mu_{\text {leff }} \approx \mu_{\text {eff }}$, i.e. that both cables experience the same coefficient of friction when in contact with the same pulley. Making this assumption reduces equation (4-14) to 


$$
\log \left(\frac{\tau_{1}}{\tau_{1}+\mathrm{m}}\right)+(1+\mathrm{GF}) \mathrm{m}+2 \mathrm{GF}\left(\tau_{1}-1\right)=0
$$

where we now define GF as

$$
\mathrm{GF} \equiv \frac{\mathrm{L} \mu_{\mathrm{eff}}}{\mathrm{R}}
$$

Solving equation (4-15) for $\tau_{1}$ gives the dimensionless equilibrium tension in the lower cable when the dimensionless applied moment is $\mathrm{m}$. Note that GF alone determines relationship between $\tau_{1}$ and $\mathrm{m}$. Unfortunately we cannot solve explicitly for $\tau_{1}$ so we must resort to solving for it numerically. Once we have, we find $\tau_{h}$ by substituting $\tau_{1}$ into the equilibrium constraint (4-4) to get

$$
\tau_{\mathrm{h}}=\tau_{1}+\mathrm{m}
$$

In Section 4.2.5 we solve for $\tau_{\mathrm{l}}$ and $\tau_{\mathrm{h}}$ for a variety of GF values, presenting the results in Figure 4.8. and Figure 4.9.

\section{Equilibrium Deflection:}

Once we have the equilibrium tensions we find the equilibrium deflection by substituting either of the constitutive relations (4-5) or (4-6) into equation (4-2) to get

$$
\theta=\frac{\mathrm{T}_{0}}{\mathrm{EA} \mu_{\mathrm{eff}}}\left[\left(\tau_{\mathrm{h}}-1-\log \tau_{\mathrm{h}}\right)+\mathrm{GF}\left(\tau_{\mathrm{h}}-1\right)\right]
$$

or

$$
\theta=\frac{\mathrm{T}_{0}}{\mathrm{EA} \mu_{\mathrm{eff}}}\left[\left(\tau_{1}-1-\log \tau_{1}\right)-\mathrm{GF}\left(\tau_{1}-1\right)\right]
$$

\subsubsection{Equilibrium Equations During Unloading}

\section{Equilibrium Tensions:}

Assuming that $\mu_{\text {heff }}=\mu_{\text {leff }}=\mu_{\text {eff }}$ we find the equilibrium tensions during unloading by substituting the constitutive relations (4-9) and (4-10) into the kinematic constraint (4-1) to 
get (after some simplification)

$$
\frac{\mathrm{T}_{0}}{\mathrm{EA} \mu_{\mathrm{eff}}}\left(2\left(\sqrt{\tau_{\mathrm{h}_{\mathrm{pk}}} \tau_{\mathrm{h}}}-\sqrt{\tau_{\mathrm{l}_{\mathrm{pk}} \tau_{1}}}\right)+\tau_{\mathrm{l}}-\tau_{\mathrm{h}}+\log \frac{\tau_{\mathrm{l}_{\mathrm{pk}}}}{\tau_{\mathrm{h}_{\mathrm{pk}}}}\right)+\frac{\mathrm{T}_{0} \mathrm{~L}}{\mathrm{EAR}}\left(\tau_{\mathrm{h}}+\tau_{1}-2\right)=0
$$

where we obtain $\tau_{\mathrm{h}_{\mathrm{pk}}}$ and $\tau_{\mathrm{l}_{\mathrm{pk}}}$ by solving the loading equation (4-15) when $\mathrm{m}=\mathrm{m}_{\mathrm{pk}}$. Dividing through by $\frac{T_{0}}{E A \mu_{\text {eff }}}$ and substituting $G F=\frac{L \mu_{\text {eff }}}{R}$ this becomes

$$
2\left(\sqrt{\tau_{\mathrm{h}_{\mathrm{pk}}} \tau_{\mathrm{h}}}-\sqrt{\tau_{\mathrm{l}_{\mathrm{pk}}} \tau_{\mathrm{l}}}\right)+\tau_{1}-\tau_{\mathrm{h}}+\log \frac{\tau_{\mathrm{l}_{\mathrm{pk}}}}{\tau_{\mathrm{h}_{\mathrm{pk}}}}+\mathrm{GF}\left(\tau_{\mathrm{h}}+\tau_{1}-2\right)=0
$$

We now use the equilibrium constraint (4-4) to eliminate $\tau_{\mathrm{h}}$ which leaves

$$
2\left(\sqrt{\tau_{h_{p k}}\left(\tau_{1}+m\right)}-\sqrt{\tau_{l_{p k}} \tau_{1}}\right)-m+\log \frac{\tau_{l_{p k}}}{\tau_{h_{p k}}}+G F\left(2 \tau_{1}+m-2\right)=0
$$

Solving equation (4-22) for $\tau_{1}$ yields the dimensionless equilibrium tension in the lower cable as we reduce the dimensionless moment $\mathrm{m}$ from its peak value $\mathrm{m}_{\mathrm{pk}}$. Once we obtain a value we use the equilibrium constraint (4-4) to find that the corresponding tension $\tau_{h}$ equals

$$
\tau_{\mathrm{h}}=\tau_{1}+\mathrm{m}
$$

We consider these results in further detail in Sections 4.2.3 and 4.2.4.

\section{Equilibrium Deflection:}

To determine the equilibrium deflection $\theta$ we substitute either of the constitutive relations (4-24) or (4-10) into equation (4-2) to get

$$
\theta=\frac{\mathrm{T}_{0}}{\mathrm{EA} \mu_{\mathrm{eff}}}\left[\left(2 \sqrt{\tau_{\mathrm{h}_{\mathrm{pk}}} \tau_{\mathrm{h}}}-\tau_{\mathrm{h}}-1-\log \tau_{\mathrm{h}_{\mathrm{pk}}}\right)+\mathrm{GF}\left(\tau_{\mathrm{h}}-1\right)\right]
$$

or 


$$
\theta=\frac{\mathrm{T}_{0}}{\mathrm{EA} \mu_{\mathrm{eff}}}\left[\left(2 \sqrt{\tau_{\mathrm{l}_{\mathrm{pk}}} \tau_{1}}-\tau_{1}-1-\log \tau_{\mathrm{l}_{\mathrm{pk}}}\right)-\mathrm{GF}\left(\tau_{1}-1\right)\right]
$$

where $\tau_{1}$ and $\tau_{h}$ are found from equations (4-22) and (4-23) respectively.

\subsubsection{Special Case I: Widely Separated Pulleys $(\mathrm{GF} \approx \infty)$}

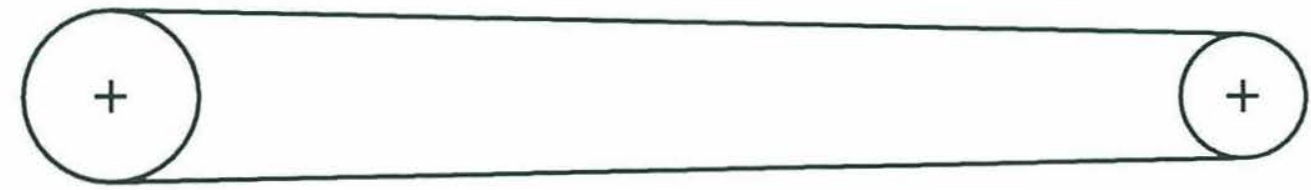

Figure 4.2. Closed Circuit Drive with Widely Separated Pulleys

When $\mathrm{GF} \approx \infty$ we can solve explicitly for the equilibrium cable tensions in the drive. A large GF value implies that $\frac{\mathrm{L}}{\mathrm{R}}$ is very large (i.e. that the drive's pulleys are very widely separated) since our experiments in Chapter 3 indicate that $\mu_{\text {eff }} \sim 0.1$. Because GF» 1 we also expect the elongation of the tangent cable to dominate the behavior of the drive. (Note: By assuming $\mathrm{GF} \approx \infty$ we will obtain the stiffness model traditionally used in the design of closed circuit cable drives (DiPietro [3]).

\section{During Loading:}

To find the equilibrium tensions we substitute $\mathrm{GF} \approx \infty$ into equation (4-15) which yields

$$
\mathrm{m}+2 \tau_{1}-2=0
$$

Solving for $\tau_{1}$ and using equation (4-23) to find $\tau_{\mathrm{h}}$ we get 


$$
\tau_{1}=1-\frac{m}{2}
$$

and

$$
\tau_{\mathrm{h}}=1+\frac{\mathrm{m}}{2}
$$

which leads to the actual tensions

$$
\begin{aligned}
& \mathrm{T}_{\mathrm{l}}=\mathrm{T}_{0}-\frac{\mathrm{M}}{2 \mathrm{R}} \\
& \mathrm{T}_{\mathrm{h}}=\mathrm{T}_{0}+\frac{\mathrm{M}}{2 \mathrm{R}}
\end{aligned}
$$

Thus the applied load affects both cables equally. From equation (4-30) we see that the low tension cable will go slack when $\mathrm{M} \geq 2 \mathrm{RT}_{0}$ or, in dimensionless terms, when

$$
\mathrm{m} \geq 2
$$

Therefore the pretension should be set to $\mathrm{T}_{0} \geq \frac{\left|\mathrm{M}_{\mathrm{pk}}\right|}{2 \mathrm{R}}$ to ensure that neither cable ever goes slack. If the low tension cable does go slack the high tension cable must counteract the entire applied moment by itself and the tension $T_{h}$ becomes

$$
\mathrm{T}_{\mathrm{h}}=\mathrm{T}_{0}+\frac{\mathrm{M}}{\mathrm{R}}
$$

or

$$
\tau_{\mathrm{h}}=1+\mathrm{m}
$$

Since GF $\approx \infty$ we drop the wrapped cable term from (4-18) and use equation (4-28) to eliminate $\tau_{\mathrm{h}}$ to get

$$
\theta=\frac{\mathrm{T}_{0} \mathrm{~L}}{2 \mathrm{EAR}} \mathrm{m}
$$

or, in terms of the actual moment $\mathrm{M}$ 


$$
\theta=\frac{\mathrm{L}}{2 \mathrm{EAR}^{2}} \mathrm{M}
$$

When $m \geq 2$ we instead use (4-33) to eliminate $\tau_{h}$ which yields

$$
\theta=\frac{\mathrm{L}}{\mathrm{EAR}^{2}} \mathrm{M}
$$

Thus we see that the stiffness drops by a factor of two when the load applied to a GF $\approx \infty$ drive goes from $m \leq 2$ to $m \geq 2$, i.e. when the lower cable goes slack. Note that both cables contribute equally to the drive's stiffness until this occurs.

\section{During Unloading:}

Assuming neither cable went slack during loading, we find the tensions during unloading by substituting $\mathrm{GF} \approx \infty$ into equation (4-22). Solving for $\tau_{1}$ we find that

$$
\tau_{1}=1-\frac{m}{2}
$$

i.e. the tensions are the same as during the loading phase.

During most of the unloading phase we can ignore the elongation of the wrapped cable, in which case we find the deflection to be

$$
\theta=\frac{\mathrm{T}_{0} \mathrm{~L}}{2 \mathrm{EAR}} \mathrm{m}=\frac{\mathrm{L}}{2 \mathrm{EAR}^{2}} \mathrm{M}
$$

which is also the same as the deflection for the loading case. However, equation (4-38) predicts that the drive will have no hysteresis deflection once we fully remove the load. In reality tension bumps must form in the wrapped sections of cable which means that the drive cannot fully return to its original position. To find the final hysteresis angle we substitute the peak load $\tau_{\mathrm{h}_{\mathrm{pk}}}=1+\frac{\mathrm{m}_{\mathrm{pk}}}{2}$ and the final load $\tau_{\mathrm{h}}=1$ into equation (4-24) to get the hysteresis angle for the drive after the load is removed

$$
\theta=\frac{\mathrm{T}_{0}}{\mathrm{EA} \mu_{\mathrm{eff}}}\left[\left(2 \sqrt{\left(1+\frac{\mathrm{m}_{\mathrm{pk}}}{2}\right)}-2-\log \left(1+\frac{\mathrm{m}_{\mathrm{pk}}}{2}\right)\right)\right]
$$




\subsubsection{Special Case II: Narrowly Separated Pulleys $(G F \approx 0)$}

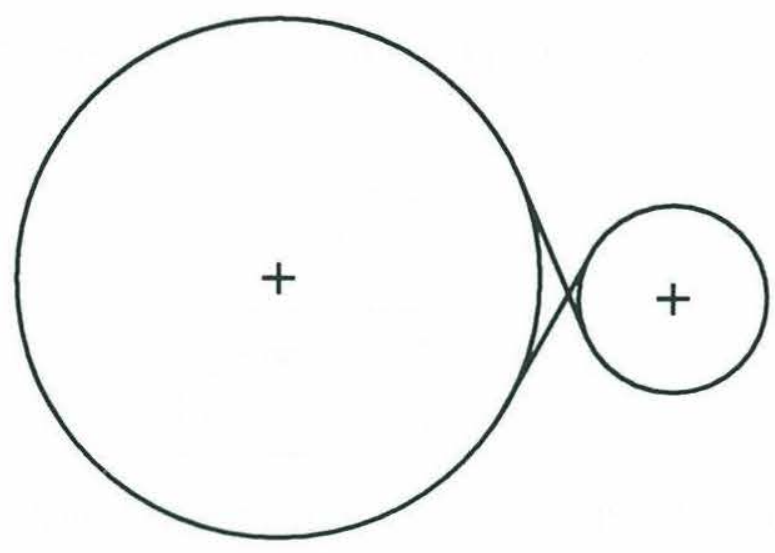

Figure 4.3. Closed Circuit Drive with Narrowly Separated Pulleys

We can also find closed form expressions for the equilibrium cable tensions when $\mathrm{GF} \approx 0$. A small GF value implies that $\frac{\mathrm{L}}{\mathrm{R}}$ is small (i.e. that the drive's pulleys are close together) since we expect that $\mu_{\mathrm{eff}} \sim 0.1$. Because GF « 1 we expect the elongation of the wrapped cable to account for most of the drive's deflection under load.

\section{During Loading:}

To find the equilibrium tensions during loading we substitute $\mathrm{GF} \approx 0$ into equation (4-15) which leads to

$$
\log \left(\frac{\tau_{1}}{\tau_{1}+m}\right)+m=0
$$

Taking the exponential of both terms and rearranging we get

$$
\tau_{1}\left(1-\mathrm{e}^{-\mathrm{m}}\right)=\mathrm{me}^{-\mathrm{m}}
$$

Solving for $\tau_{1}$ we find 


$$
\tau_{1}=\frac{\mathrm{me}^{-\mathrm{m}}}{1-\mathrm{e}^{-\mathrm{m}}}=\frac{\mathrm{m}}{\mathrm{e}^{\mathrm{m}}-1}
$$

Using this result to eliminate $\tau_{1}$ from the equilibrium constraint (4-17) we find that

$$
\begin{gathered}
\tau_{\mathrm{h}}=\mathrm{m}\left(1+\frac{1}{\mathrm{e}^{\mathrm{m}}-1}\right) \\
\tau_{\mathrm{h}}=\frac{\mathrm{me}}{\mathrm{e}^{\mathrm{m}}-1}
\end{gathered}
$$

By symmetry we expect the tensions to be reversed when we apply a negative moment, i.e. that $\tau_{1}(-m)=\tau_{h}(m)$ and $\tau_{h}(-m)=\tau_{l}(m)$. By substituting $-m$ for $m$ in equations (4-42) and (4-44) and simplifying we find that this is true. Therefore these results hold for both positive and negative loads.

To find the elongation we use equation (4-44) to eliminate $\tau_{\mathrm{h}}$ from (4-18) to get

$$
\theta=\frac{\mathrm{T}_{0}}{\mathrm{EA} \mu_{\text {eff }}}\left(\left(\frac{\mathrm{me}^{\mathrm{m}}}{\mathrm{e}^{\mathrm{m}}-1}-1\right)(1+\mathrm{GF})-\log \frac{\mathrm{me}}{\mathrm{e}^{\mathrm{m}}-1}\right)
$$

Notice that we have not dropped GF from the equation even though GF $\approx 0$. Keeping GF in the equation ensures that the model accurately predicts the stiffness when $\mathrm{m}$ is small. To find the stiffness of the drive we determine $\frac{\mathrm{dm}}{\mathrm{d} \theta}$ which (leaving out many steps) equals

$$
\mathrm{k}_{\mathrm{T}}=\frac{\mathrm{dm}}{\mathrm{d} \theta}=\frac{\mathrm{EA} \mu_{\text {heff }}}{\mathrm{T}_{0}}\left(\frac{\left(\mathrm{e}^{\mathrm{m}}-1\right)^{2}}{\mathrm{e}^{\mathrm{m}}\left(\mathrm{e}^{\mathrm{m}}-\mathrm{m}-1\right)}\right)\left(1+\mathrm{GF}-\frac{\mathrm{e}^{\mathrm{m}}-1}{\mathrm{me}}\right)^{-1}
$$

Taking the limit as $m \rightarrow 0$ we find that

$$
\left.\mathrm{k}_{\mathrm{T}}\right|_{\mathrm{m}=0}=\frac{\mathrm{EA} \mu_{\text {heff }}}{\mathrm{T}_{0}} \frac{2}{\mathrm{GF}}=\frac{2 \mathrm{EAR}}{\mathrm{L}}
$$

i.e. the zero load stiffness is due entirely to the tangent lengths of cable since the stiffness of the wrapped cable is infinite at this point. 
Modelling: Special Case II: Narrowly Separated Pulleys

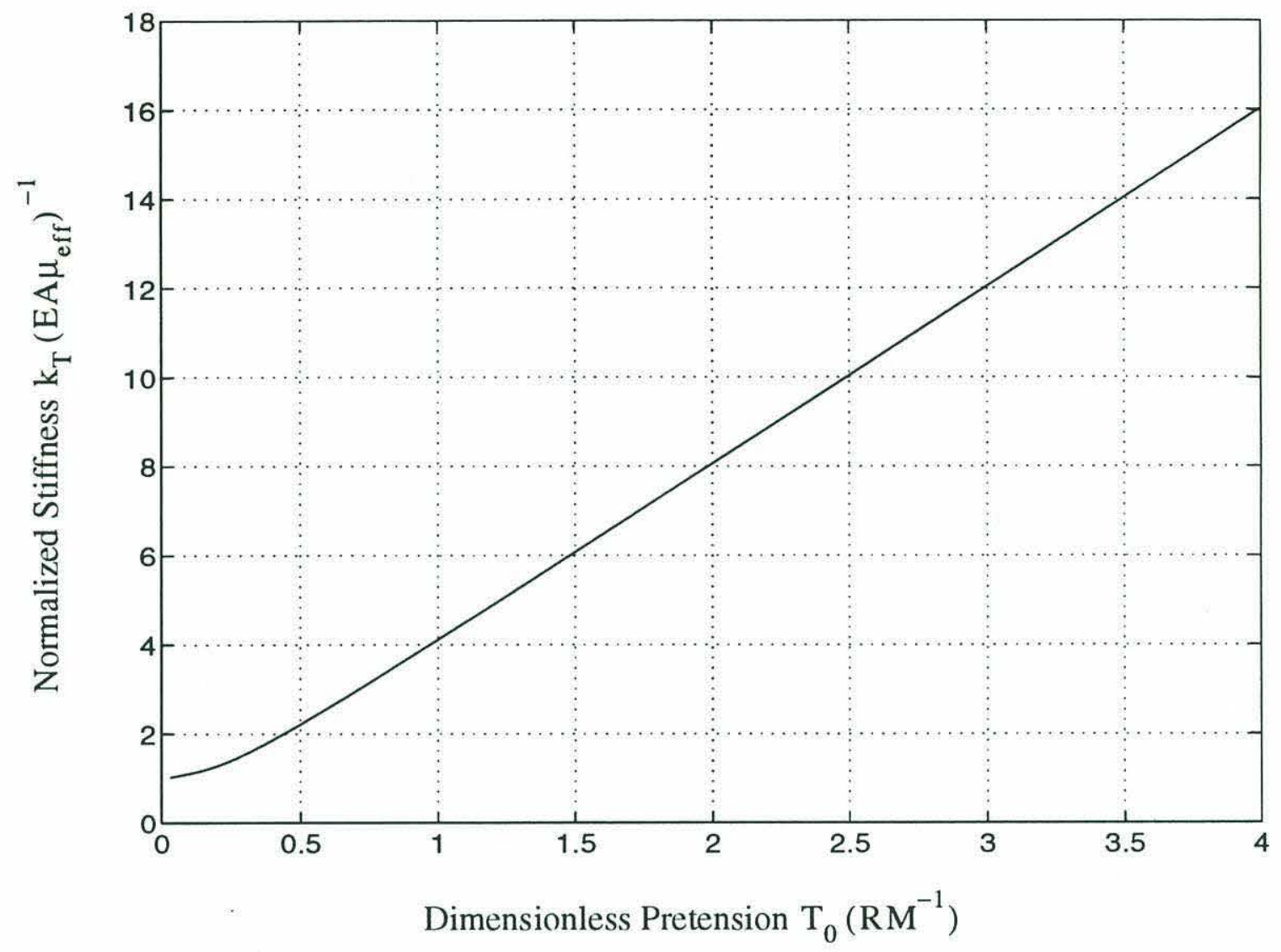

Figure 4.4. Fixed Load Stiffness vs. Initial Pretension

We typically want to maximize the stiffness of a cable drive. Figure 4.4. shows the normalized stiffness $\mathrm{k}_{\mathrm{T}}\left(\mathrm{EA} \mu_{\mathrm{eff}}\right)^{-1}$ as the dimensionless pretension $\mathrm{T}_{0}\left(\mathrm{RM}^{-1}\right)$ (the dimensionless pretension is equivalent to the inverse of the dimensionless moment $\mathrm{m}$ ). For a fixed load $M$ this plot shows how the drive's stiffness changes when we vary $\mathrm{T}_{0}$. We see that above $\mathrm{T}_{0}\left(\mathrm{RM}^{-1}\right) \approx 0.5$ the stiffness increases more or less linearly with pretension. Thus we must make a trade-off when selecting a pretension for a drive. High preloads give higher stiffness and delay the point at which the low tension cable goes slack. However, high preloads also reduce cable fatigue life (by increasing the cable stress) and also increase bearing friction. (For information on cable fatigue life see the SAVA Industries, Inc. miniature cable catalog [10]) 


\section{During Unloading:}

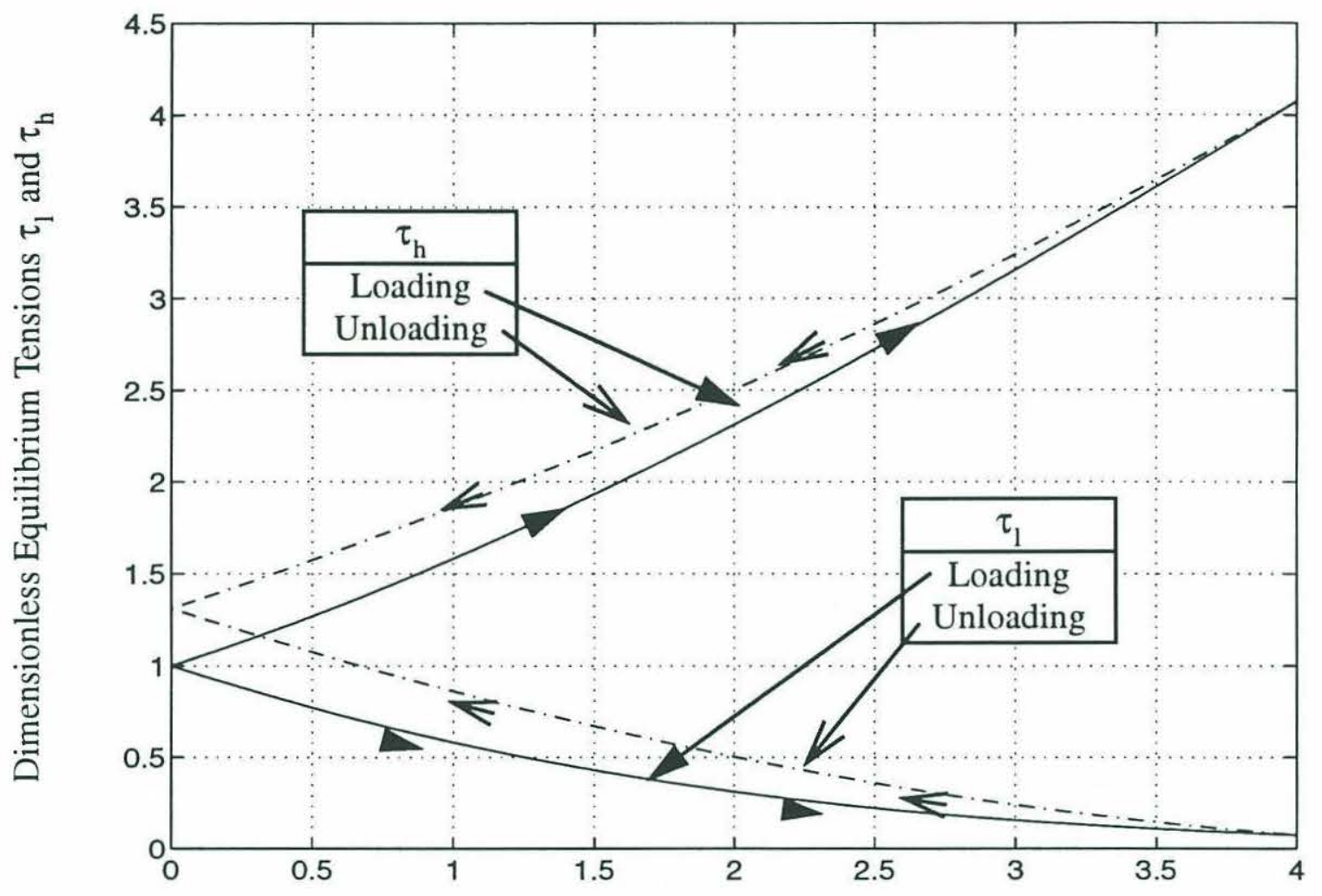

Dimensionless Moment m

Figure 4.5. Equilibrium Tensions During Loading and Unloading when $\mathrm{GF}=0$

Figure 4.5. shows the equilibrium tensions $\tau_{1}$ and $\tau_{\mathrm{h}}$ as we apply and then remove a load of $\mathrm{m}_{\mathrm{pk}}=4$ from the drive (see Appendix $\mathrm{C}$ for the derivation of the tensions $\tau_{1}$ (equation (D-18)) and $\tau_{\mathrm{h}}$ (equation (D-20)) during unloading). Notice that the final tension exceeds the initial pretension. As we load the drive four affected angles of wrap develop, one in each of the four sections of wrapped cable (see Figure 4.6.). When we remove the load, two tension bumps form in the upper half of the circuit while two tension dips form in the lower half. The cable in these regions remains elongated (in the bumps) or contracted (in the dips) after we remove the load. Since the total distance traversed by the cable has not changed, the cable in the tangent sections must make up the difference, which can only occur if the final tension is different from the initial tension. As we shall see in the next section, the affected angles of wrap in the low tension cable are always larger than the affected angles of wrap in the high tension cable. As a result the net change in length of the wrapped cable is negative, requiring that the tangent sections elongate, which in turn requires that the final tension be higher than the initial tension. 


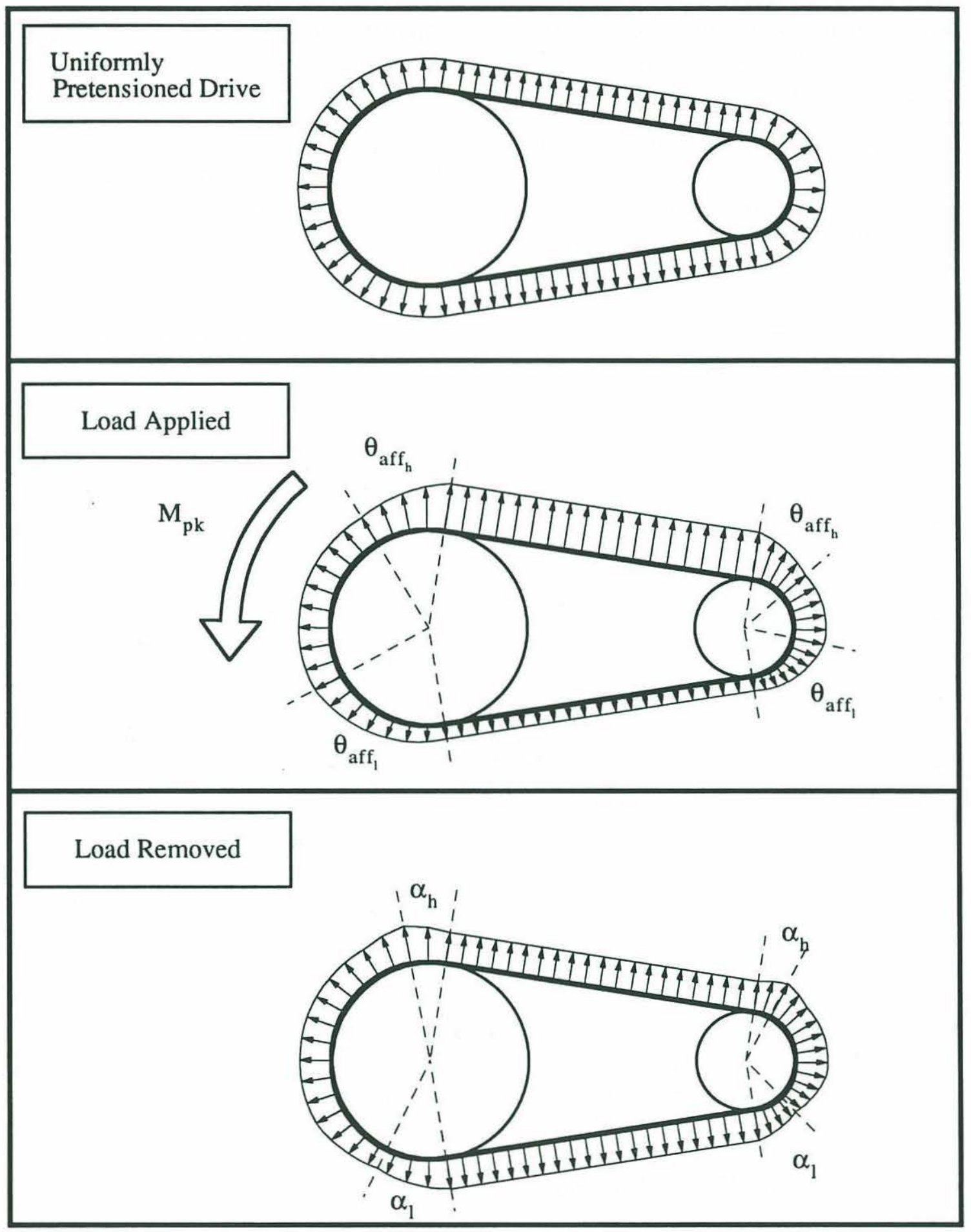

Figure 4.6. Affected Angles of Wrap and Tension Bump Locations 


\section{Chapter 4: Closed Circuit Cable/Pulley Drives}

The solid curves in Figure 4.7. show the deflections associated with the exact solutions for the cable tensions for the loading/unloading cycle shown in Figure 4.5. The dashed curve shows the deflection obtained when we approximate the tension during unloading as being the same as the tension during loading (i.e. when we use equations (4-42) and (4-44) approximate the tensions during unloading). For this loading case $\left(m_{p k}=4\right)$ the error in the final hystseresis deflection (i.e. when $\mathrm{m}=0$ ) is roughly $30 \%$. For smaller peak loads this error would be smaller. Thus we see that the approximate solution yields a passable estimate of the expected hysteresis angle for a drive while avoiding the considerable additional effort required to find the exact solution.

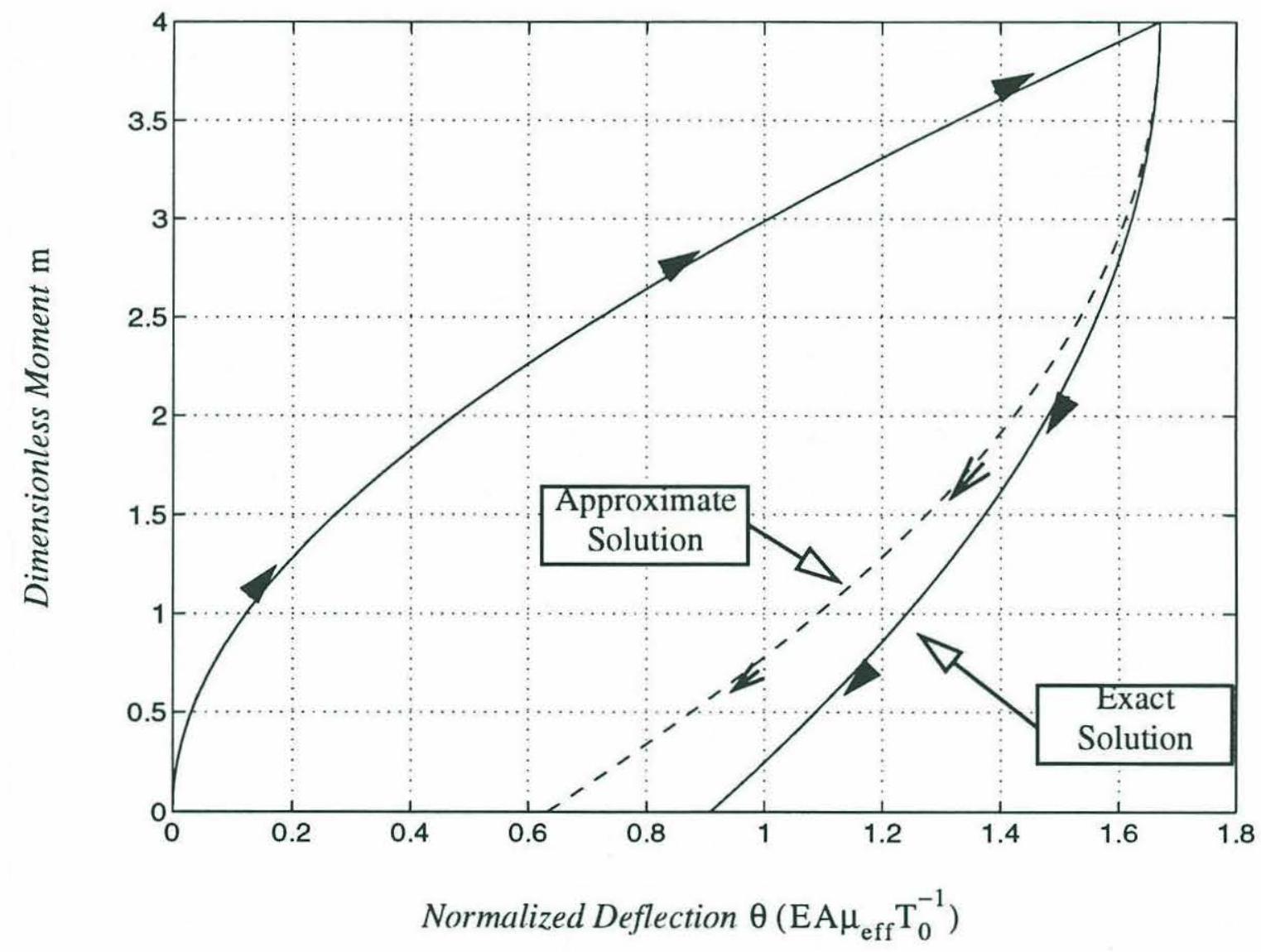

Figure 4.7. Equilibrium Deflections During Loading and Unloading When $\mathrm{GF}=0$

\subsubsection{General Case Solutions}

When a drive's GF value lies between 0 and $\infty$ we must resort to solving the equilibrium equations numerically. We focus primarily on the deflection during loading. 
Modelling: General Case Solutions

\section{Equilibrium Tensions During Loading:}

Figure 4.8. shows $\tau_{1}$ as a function of $m$ for a number of different GF values. In Section 4.2.3 we found that when GF $=\infty$ the low tension cable goes slack for any load over $\mathrm{m}=2$ and showed that a pretension $\mathrm{T}_{0} \geq \frac{\mathrm{M}_{\max }}{2 \mathrm{R}}$ would prevent this from occurring.

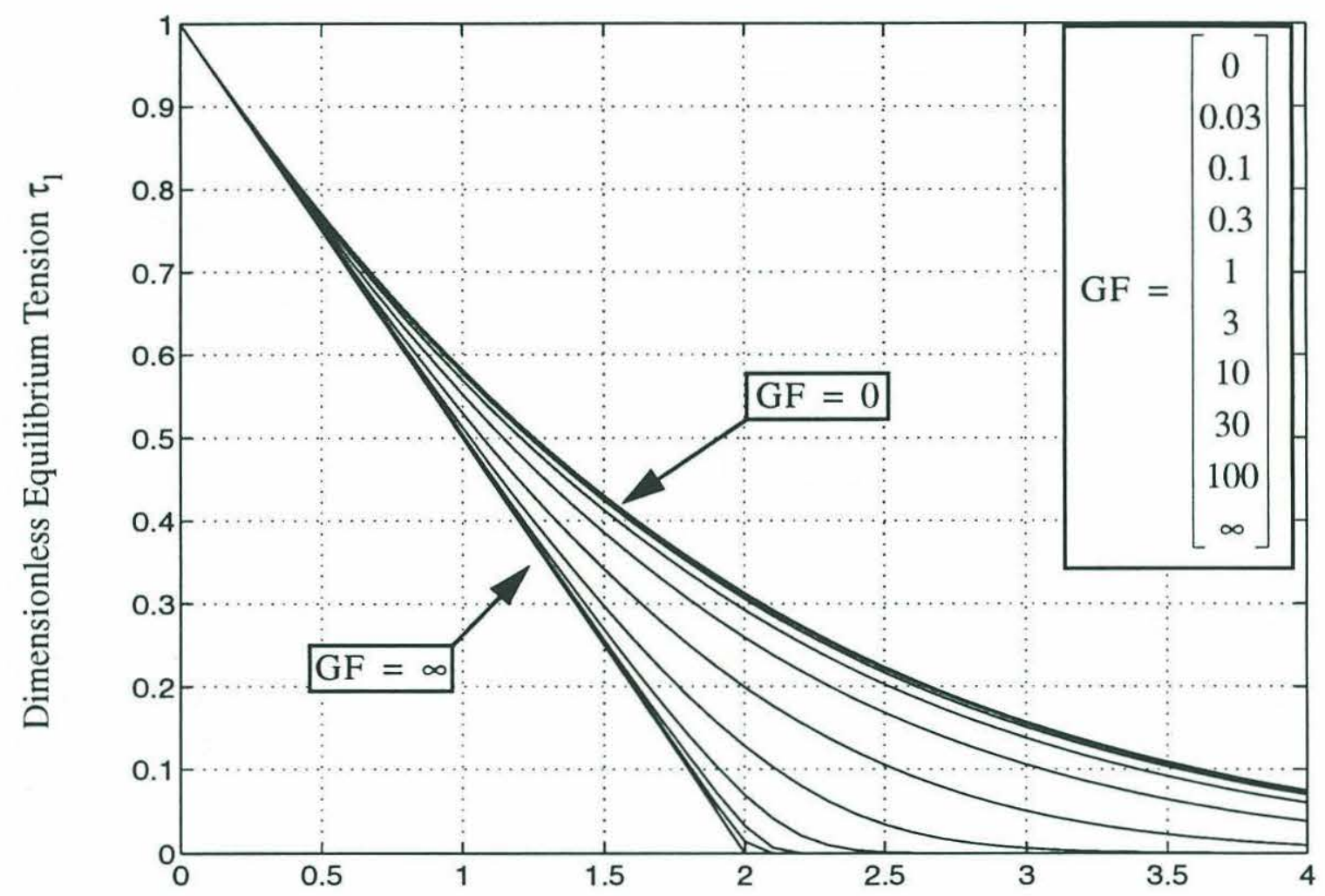

Dimensionless Moment m

Figure 4.8. Dimensionless Tension $\tau_{1}$ vs. Dimensionless Moment $\mathrm{m}$, Various Values of GF

Since drives with lower GF values do not go slack as quickly, the minimum pretension required to prevent slip is lower. For example, if GF $=0$ we could select $T_{0} \geq \frac{M_{\max }}{4 R}$ and still not go slack at maximum load (note that $\mathrm{m}_{\max }=4$ when we use this pretension). By reducing the pretension used we can reduce bearing friction and, by reducing the cable stress, can increase the fatigue life of the drive. However, as we showed in Section 4.2.4, reductions in pretension also decrease the stiffness of the drive. All of these issues must be 


\section{Chapter 4: Closed Circuit Cable/Pulley Drives}

considered when tailoring a cable drive to a particular application.

Figure 4.9. shows the corresponding tension in the high tension cable for various GF values. As we showed in Section 4.2.3 the slope of the GF $=\infty$ solution doubles at $\mathrm{m}=2$ as the low tension cable goes slack and the high tension cable must support the applied load by itself. In both the $\tau_{1}$ and $\tau_{\mathrm{h}}$ plots the GF $=\infty$ tensions define the asymptotes of the all the other solutions.

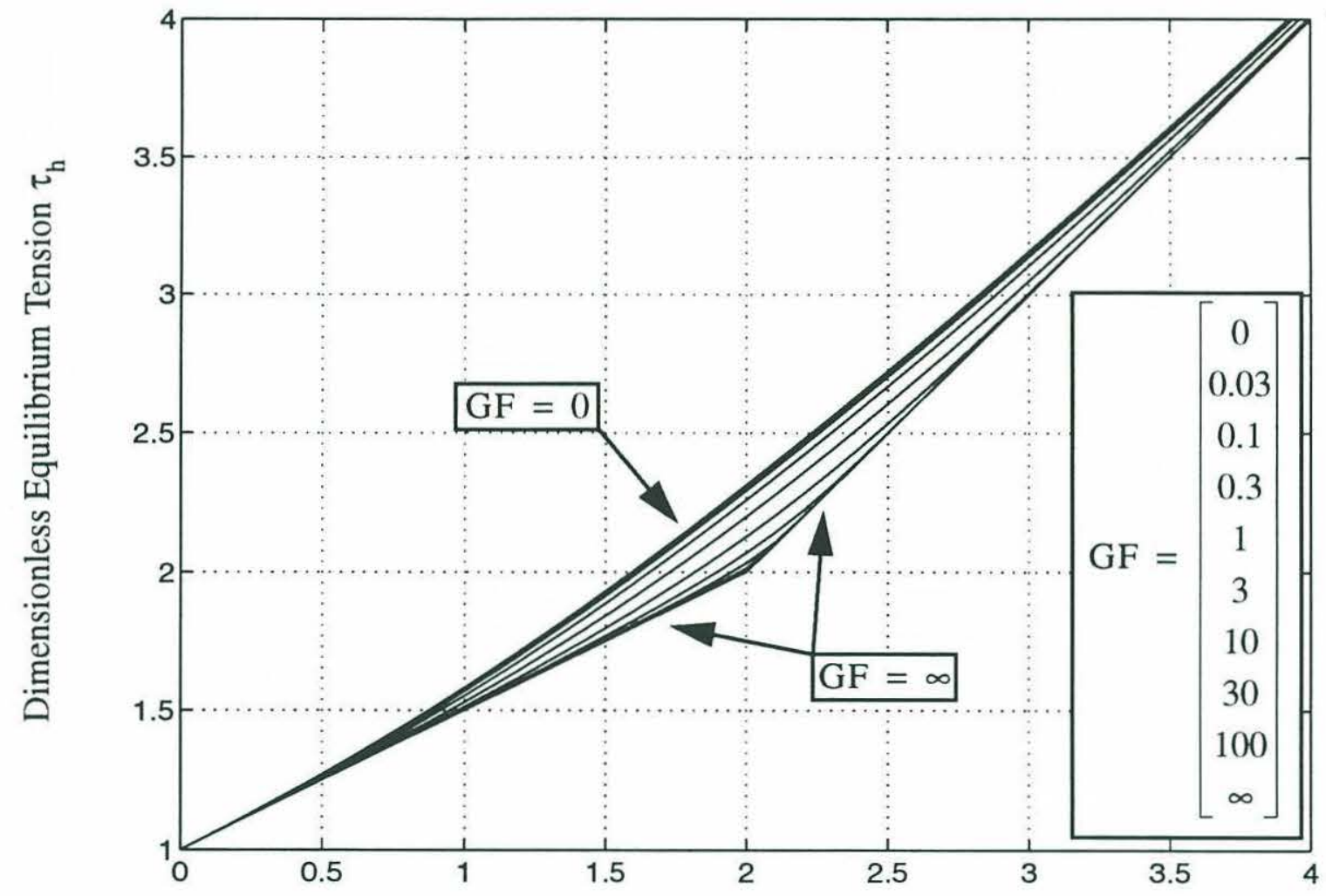

Dimensionless Moment m

Figure 4.9. Dimensionless Tension $\tau_{\mathrm{h}}$ vs. Dimensionless Moment $\mathrm{m}$, Various Values of $\mathrm{GF}$

In Chapter 3 we found that a negative moment (one that tends to make the cable go slack) causes the stiffness of an open circuit drive to drop off more rapidly than would an equivalent positive moment (see Figure 3.3.). Therefore the upper half of the closed circuit drive will be stiffer than the lower half and as such must bear a higher share of the load, with the difference depending on the value of GF. This is why $\tau_{1}$ drops off less rapidly quickly for drives with low GF values. As GF increases the difference in stiffness between the two halves of the closed circuit drive becomes less pronounced, causing $\tau_{1}$ to drop off more quickly. 


\section{Affected Angles of Wrap:}

Although each of the $\tau_{1}$ curves in Figure 4.8. approaches zero tension asymptotically the actual tension in a real drive would eventually reach zero. This discrepancy stems from our assumption that the affected angle of wrap never exceeds the actual angle of wrap. A real drive has a finite angle of wrap, so we can always load it severely enough to make the cable go slack.
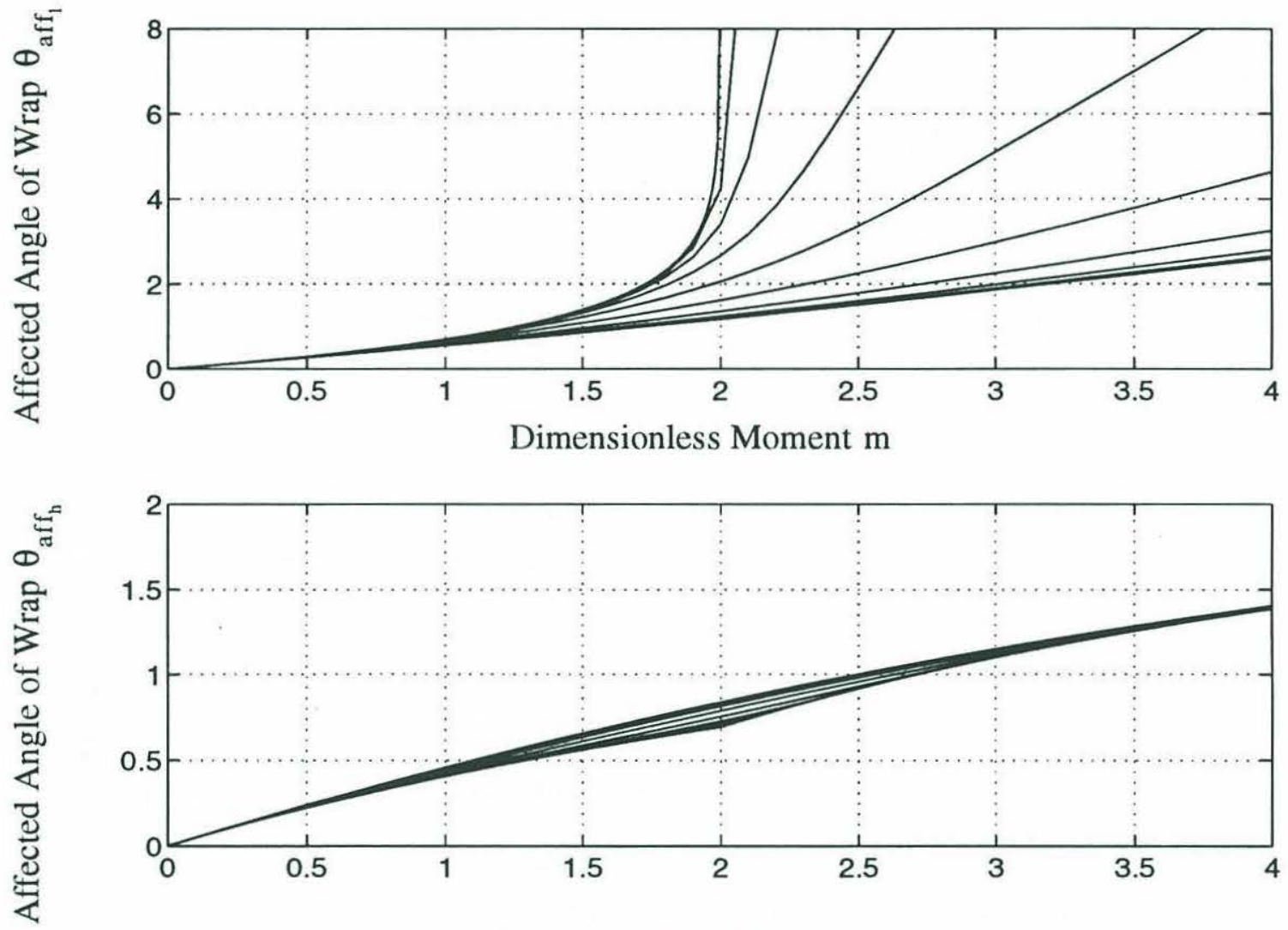

Dimensionless Moment m

Figure 4.10. Normalized Affected Angles of Wrap vs. Dimensionless Moment $\mathrm{m}$

Figure 4.10. shows the normalized affected angles as a function of $\mathrm{m}$ for the high and low tension halves of the circuit. Note that the affected angle of wrap for the low tension cable always exceeds the affected angle in the high tension cable. Knowing the maximum applied moment and the GF value for a drive we can use this plot to find the maximum affected angle of wrap. By designing the drive such that the available angle of wrap always exceeds the affected angle of wrap we help ensure that neither cable ever goes slack. To do this we take the angle of wrap required for the desired range of motion and add to it the amount $\theta_{\text {aff }_{1}}$. Note that we do this for both pulleys. 


\section{Chapter 4: Closed Circuit Cable/Pulley Drives}

\section{Equilibrium Deflection:}

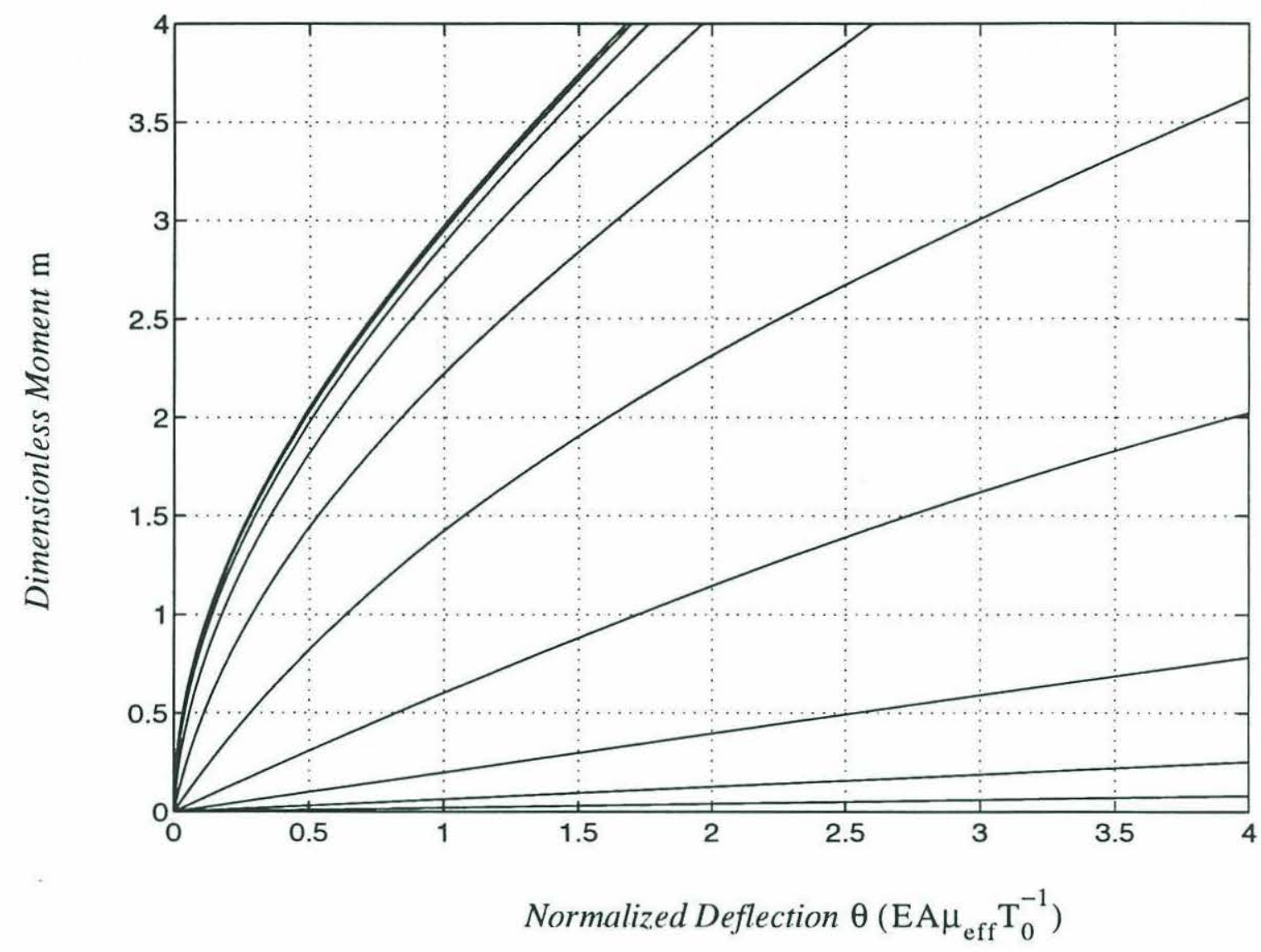

Figure 4.11.Dimensionless Moment $\mathrm{m}$ vs.Normalized Deflection $\theta\left(\mathrm{EA} \mu_{\mathrm{eff}} \mathrm{T}_{0}^{-1}\right)$

Figure 4.11.and Figure 4.12. show, at different scales, the dimensionless moment as a function of the normalized deflection for drives with various GF values. The initial slope of every curve (i.e. initial stiffness of the drive) equals $\left.\frac{\mathrm{dm}}{\mathrm{d} \theta}\right|_{m=0}=\frac{2}{\mathrm{GF}}=\frac{2 \mathrm{EAR}^{2}}{\mathrm{~L}}$. For very small loads the wrapped cable appears to be infinitely stiff which means that the tangent sections of cable alone define the initial stiffness. Figure 4.11. shows that low GF drives are always stiffer than high GF drives. However, the stiffness of a low GF drive depends more strongly on the applied load than does the stiffness of a drive with a higher GF value. Also, as shown in section 4.2.4, the stiffness of low GF drives increases if we increase the pretension $\mathrm{T}_{0}$. In section $s 4.2 .3$ we saw that the stiffness of high GF drives is effectively independent of the pretension. 


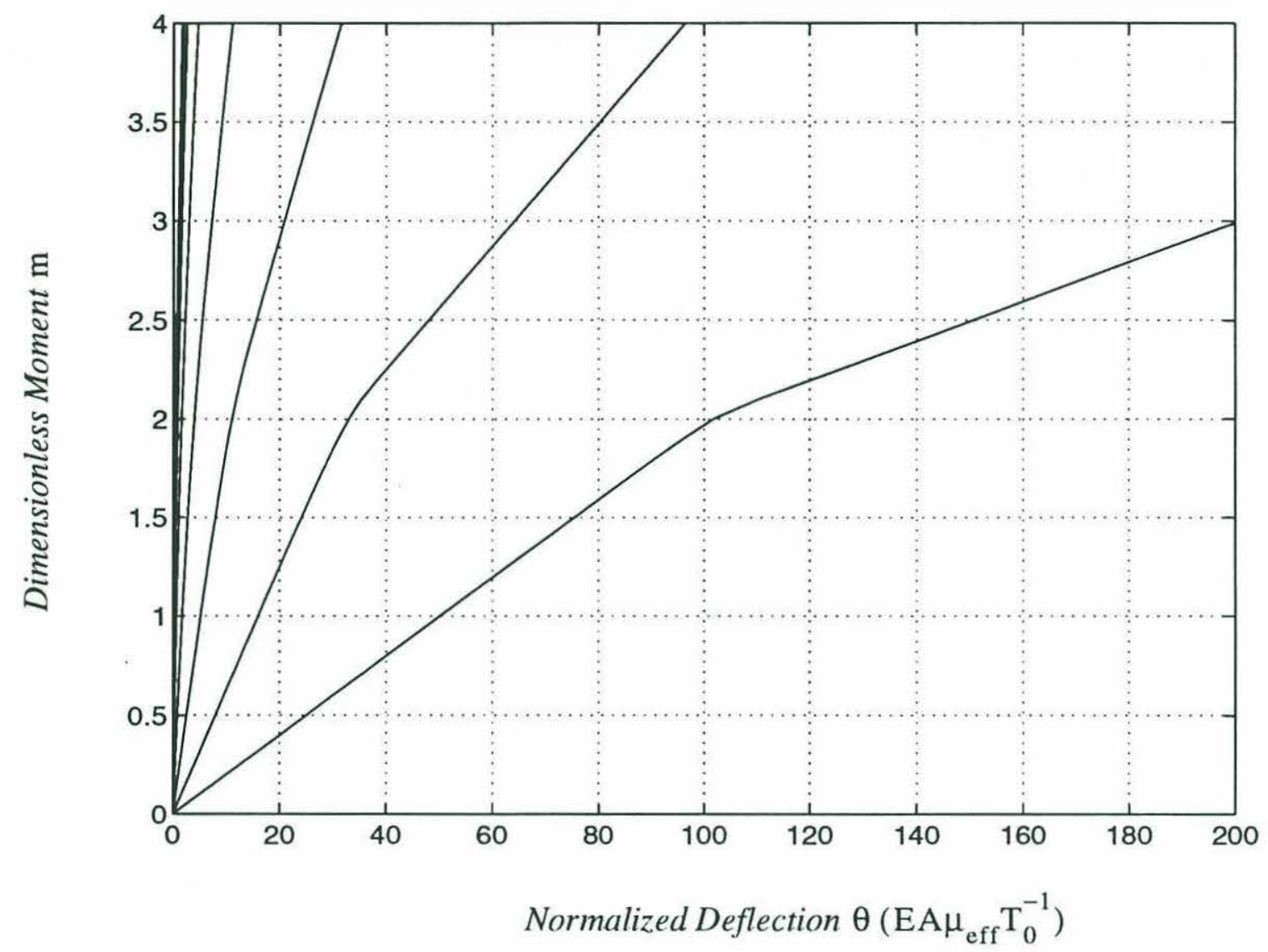

Figure 4.12. Dimensionless Moment $\mathrm{m}$ vs.Normalized Deflection $\theta\left(\mathrm{EA} \mu_{\mathrm{eff}} \mathrm{T}_{0}^{-1}\right)$.

\subsubsection{Approximate Solutions for the General Case}

We expect that for some range of GF values we can use the GF $=\infty$ and GF $=0$ solutions to approximate the actual tensions in drives having other GF values. To identify over what range of GF values these approximations hold we compare the deflections based on these approximate solutions to numerically obtained exact solutions. Focusing on the deflection during loading we investigate three different approximations, starting in each case with the deflection equation (4-18), which we repeat here

$$
\theta=\frac{\mathrm{T}_{0}}{\mathrm{EA} \mu_{\mathrm{eff}}}\left[\left(\tau_{\mathrm{h}}-1-\log \tau_{\mathrm{h}}\right)+\mathrm{GF}\left(\tau_{\mathrm{h}}-1\right)\right]
$$




\section{Model I: GF $=\infty$ Tensions, Neglect Wrap Deflection Term}

In this case we use the GF $=\infty$ tension solution $\tau_{\mathrm{h}}=1+\frac{\mathrm{m}}{2}$ to approximate the equilibrium tension in the upper cable. Since GF » 1 we also neglect the wrap deflection term from (4-18) to get

$$
\theta=\frac{\mathrm{L}}{\mathrm{EAR}^{2}} \mathrm{M}
$$

Figure 4.13. shows the deflection error associated with this approximation for the same GF values we investigated in the previous section. Recalling that this model only applies up to $\mathrm{m}=2$ we see that the approximation is reasonable (say, less than $15 \%$ error) for GF $\geq 10$.

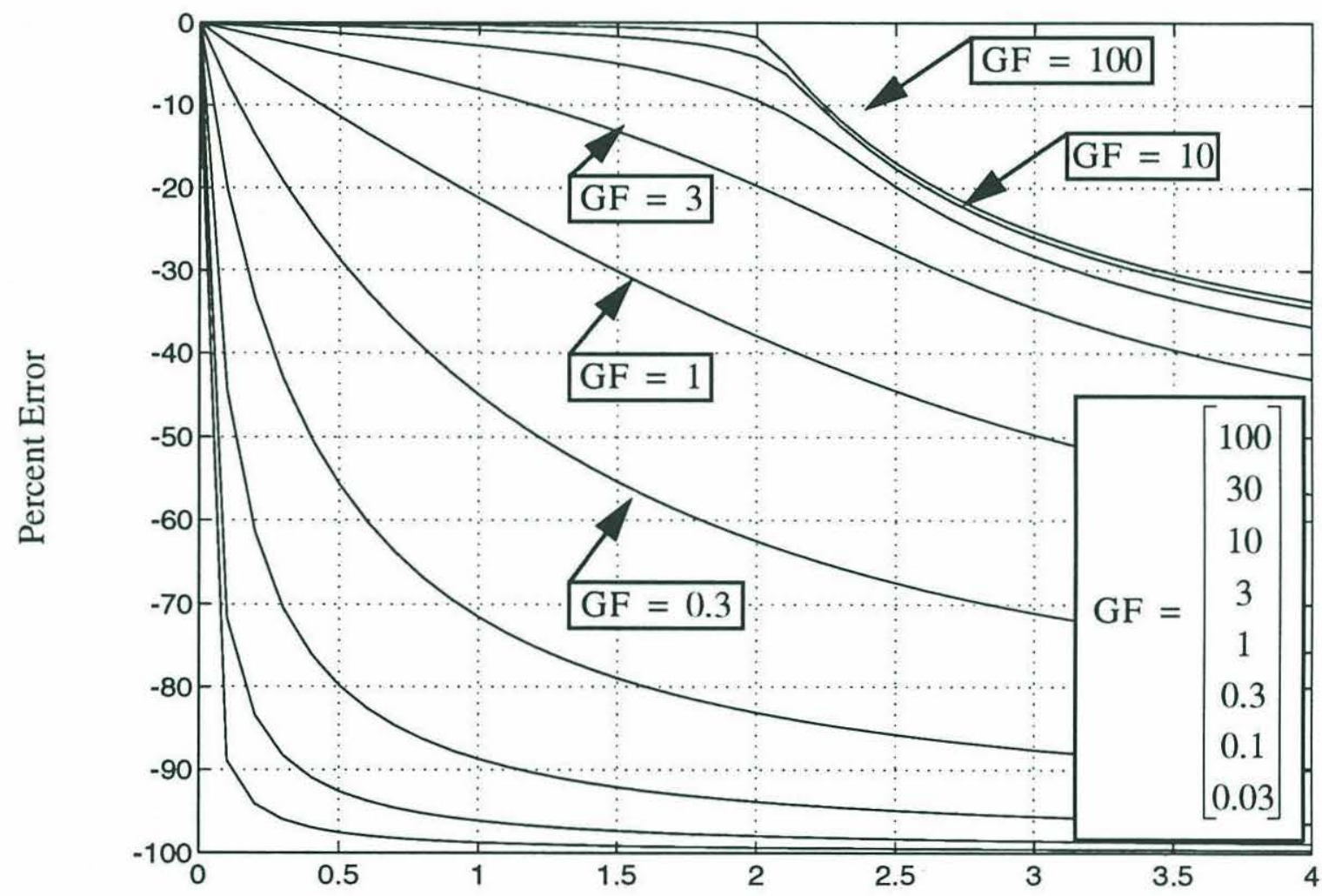

Dimensionless Moment m

Figure 4.13. Accuracy Approximate Model I without Wrapped Cable Term 


\section{Model II: GF $=\infty$ Tensions, Keep Wrap Deflection Term}

In this case we still use $\tau_{\mathrm{h}}=1+\frac{\mathrm{m}}{2}$ to approximate the equilibrium cable tension but this time we do not drop the wrap deflection term. The resulting model for the deflection is

$$
\theta=\frac{\mathrm{T}_{0}}{\mathrm{EA} \mu_{\mathrm{eff}}}\left(\frac{\mathrm{M}}{2 \mathrm{RT}}(1+\mathrm{GF})-\log \frac{\mathrm{M}}{2 \mathrm{RT}}\right)
$$

Figure 4.14. shows the deflection error during loading associated with this model. We note that overall there is a significant reduction in the error for most GF values. Recognizing that Model II also applies only to $\mathrm{m}=2$ and using $15 \%$ error as our cut-off we see that Model II gives accurate results for, say, GF $\geq 3$

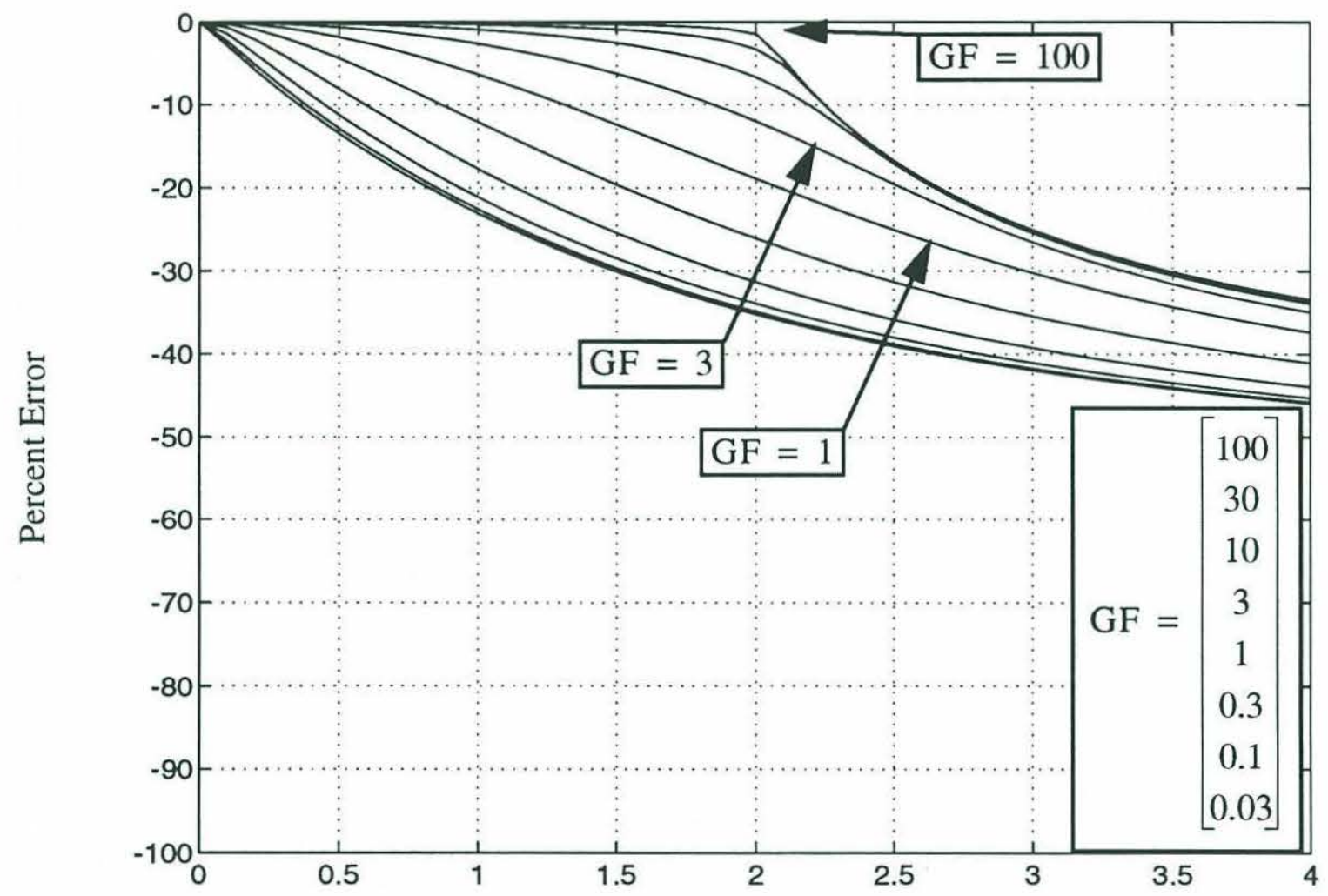

Dimensionless Moment m

Figure 4.14. Accuracy of Approximate Solution I with Wrapped Cable Term 


\section{Chapter 4: Closed Circuit Cable/Pulley Drives}

Model III: GF $=0$ Tensions

In this case we use the GF $=0$ tension solution, $\tau_{h}=\frac{m e^{m}}{e^{m}-1}$ to approximate the actual equilibrium tensions, in which case (4-18) becomes

$$
\theta=\frac{T_{0}}{E A \mu_{\text {eff }}}\left[\left(\left(\frac{m e^{m}}{e^{m}-1}-1\right)(1+G F)-\log \frac{m e^{m}}{e^{m}-1}\right)\right]
$$

Unlike the other models, this model applies beyond $\mathrm{m}=2$ because the lower cable does not go slack until well beyond this point. Figure 4.15. shows the deflection error associated with using this model. Using $15 \%$ error as the cutoff we see that the model gives accurate results when, say, GF $\leq 1$.

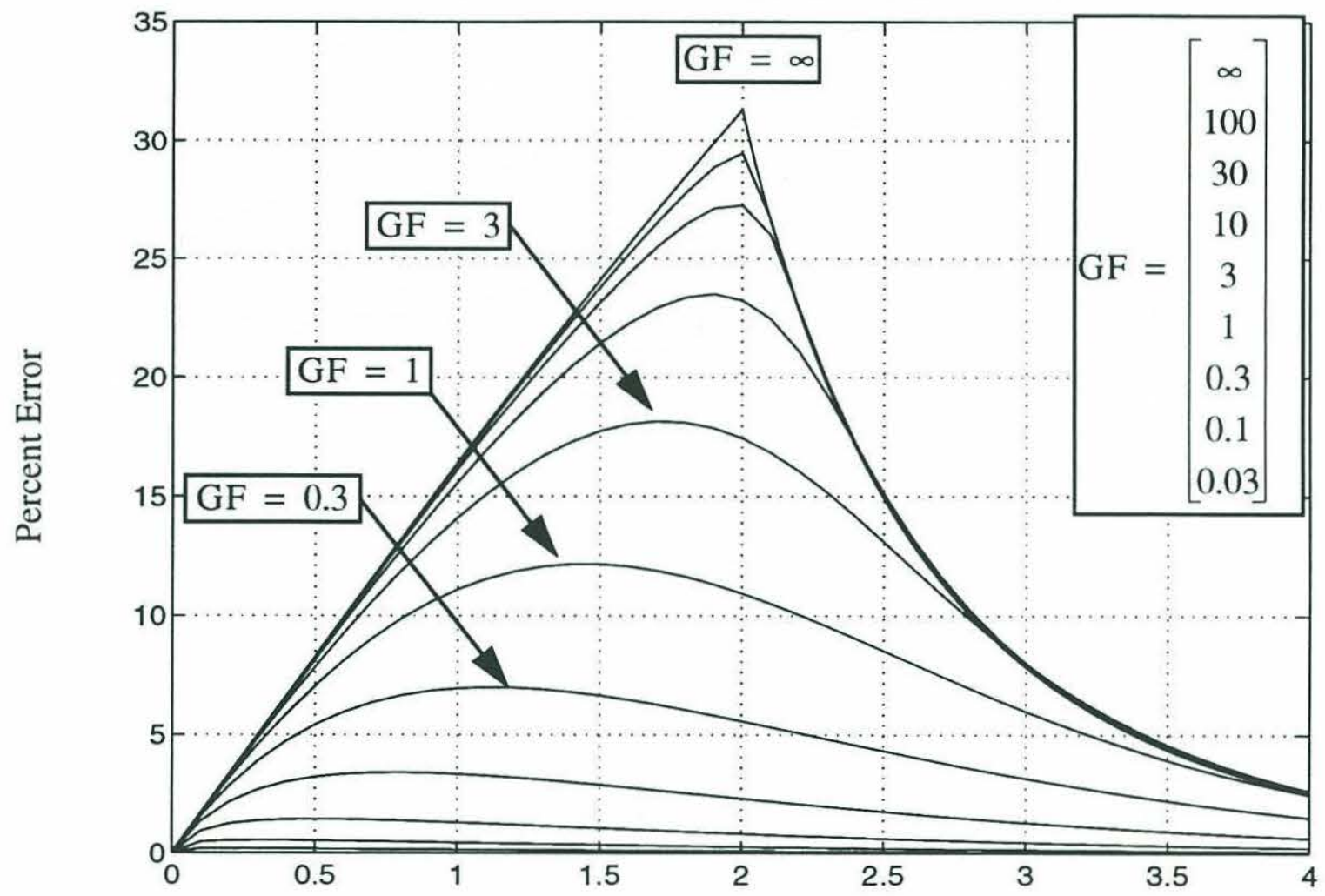

Dimensionless Moment m

Figure 4.15. Accuracy of Approximate Solution II

Thus we can accurately model almost all closed circuit drives without having to solve numerically. 


\subsection{Experimental Confirmation}

In this section we show that the Model III solution agrees closely with data obtained from experiments on an actual closed circuit drive. We briefly present the theoretical basis for the experiment and follow with a presentation and discussion of the results. For a description of the experimental apparatus and procedures used see Appendix A.

\subsubsection{Approach}

From each trial we obtain a vector of applied loads $\stackrel{9}{\mathrm{M}}$ and an associated vector of deflections $\theta$ (the " signifies that the quantity is a sequence values). Defining

$$
\mathrm{m} \equiv \frac{\stackrel{\circ}{\mathrm{RT}}}{\mathrm{RT}_{0}}
$$

and

$$
\dot{\tau}_{\mathrm{h}}=\frac{\mathrm{m} \mathrm{e}^{\stackrel{\mathfrak{m}}{ }}}{\mathrm{e}^{\dot{\mathrm{m}}}-1}
$$

we see that Model III is correct if

$$
\hat{\theta}=\frac{\mathrm{T}_{0}}{\mathrm{EA} \mu_{\text {eff }}}\left[\dot{\bullet}_{\mathrm{h}}-1-\log \dot{\tau}_{\mathrm{h}}\right]+\frac{\mathrm{LT}_{0}}{\mathrm{EAR}}\left[\dot{\tau}_{\mathrm{h}}-1\right]
$$

Defining the wrapped cable deflection $\hat{\theta}_{\mathrm{wr}}$ and the processed load vector $\mathrm{x}_{1}$ as

$$
\begin{gathered}
\hat{\theta}_{\mathrm{wr}} \equiv \hat{\theta}-\frac{\mathrm{LT}_{0}}{\operatorname{EAR}}\left[\dot{\bullet}_{\mathrm{h}}-1\right] \\
\mathrm{\ell}_{1} \equiv\left[\dot{\bullet}_{\mathrm{h}}-1-\log \dot{\tau}_{\mathrm{h}}\right]
\end{gathered}
$$

equation (4-54) becomes

$$
\hat{\theta}_{\mathrm{wr}}=\frac{\mathrm{T}_{0}}{\mathrm{EA} \mu_{\text {eff }}^{*}} \hat{\mathrm{t}}_{1}
$$


For the values of $\theta_{\mathrm{wr}}$ associated with the unloading portion of a trial we employ a similar strategy. Equation (4-24) gives the deflection during unloading which we can rewrite as

$$
\hat{\theta}_{\mathrm{wr}}=\frac{\mathrm{T}_{0}}{\mathrm{EA} \mu_{\text {eff }}^{*}} \hat{\mathrm{x}}_{\mathrm{u}}
$$

where we define the processed load vector during unloading $\ell_{u}$ as

$$
\hat{x}_{\mathrm{u}}=2 \sqrt{\dot{\tau}_{\mathrm{pk}}} \dot{\tau}-\dot{\tau}-1-\log \left(\dot{\tau}_{\mathrm{pk}}\right)
$$

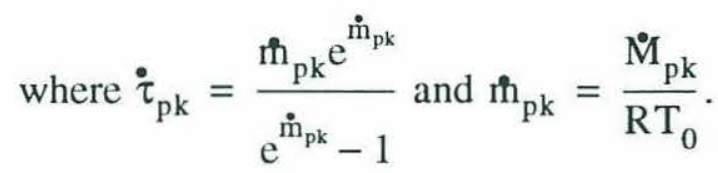

As before, we check the validity of the loading and unloading models by determining the linearity of the relationship between $\hat{\theta}_{\mathrm{wr}}$ and the appropriate processed load vector. Since the loading applied in the closed circuit tests was not fully quasi-static we compute two coefficients of friction for each a trial. We obtain the acting coefficient of friction from the slope of the line defined by $\hat{\theta}_{\mathrm{wr}}$ and the appropriate processed load vector while we determine the true coefficient of friction from the equilibrium deflections reached at the end of each half of the loading cycle. Thus our analysis of the data consists of two tasks.

1. prove the validity of the models by showing that $\hat{\theta}_{\mathrm{wr}}$ and the processed load vectors $\mathrm{x}_{1}$ and $\mathrm{x}_{\mathrm{u}}$ are linerarly related

2. determine and compare the coefficients of friction obtained from the various trials 


\subsubsection{A Typical Trial}

\section{Unprocessed Data}

Figure 4.16. shows the wrap deflection $\hat{\theta}_{\mathrm{wr}}$ and the dimensionless moment $\mathrm{m}$ as functions of time for a typical closed circuit trial. As with the open circuit trials we see that the loading is not quasi-static because the deflection continues to change after the applied load. stops changing. Therefore we shall again determine two coefficients of friction for each trial; the true coefficient of friction (determined from on the equilibrium points (A) and (B) and the acting coefficient of friction (determined from the slope of the $\hat{\theta}_{\mathrm{wr}} /$ processed load vector plots). In general the closed circuit experiments exhibited less deflection lag than the open circuit tests, particularly after the load had been removed.

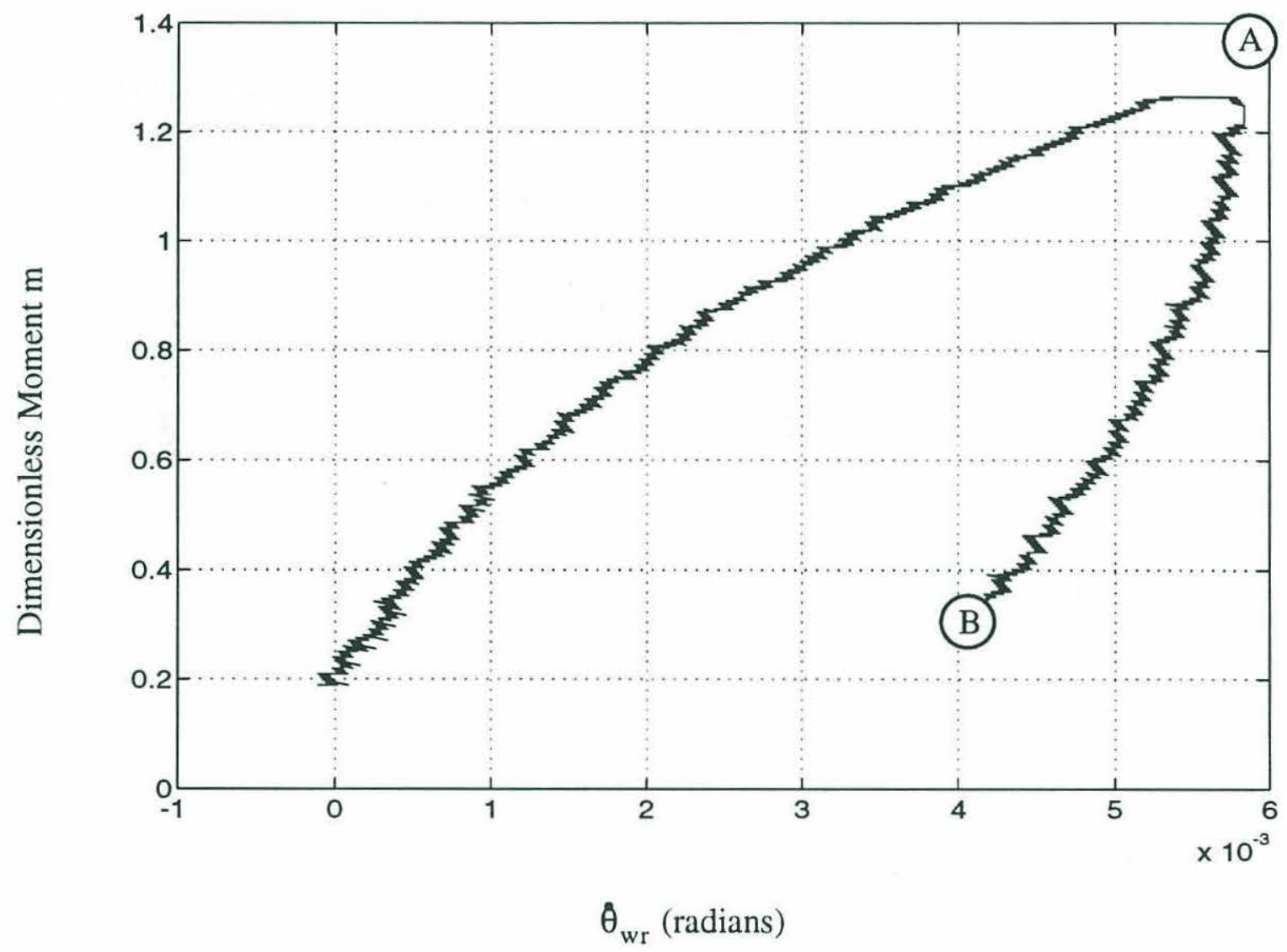

Figure 4.16. Dimensionless moment vs. Normalized Deflection, Trial 19 


\section{Chapter 4: Closed Circuit Cable/Pulley Drives}

\section{Processed Data}

Figure 4.17. shows that each of the processed load vectors $x_{1}$ and $k_{u}$ varies linearly with $\hat{\theta}_{\mathrm{wr}}$ as predicted by the loading and unloading models. To quantify the agreement we perform a least squares fit of a straight line to the data and determine the associated correlation coefficient for each curve. For this trial the acting coefficients of friction during loading and unloading are .076 and .064 and the associated R-values are .9998and .996. In addition we determine the true coefficient of friction from each equilibrium point, which yields .63 and .64 .

$\mathrm{t}$

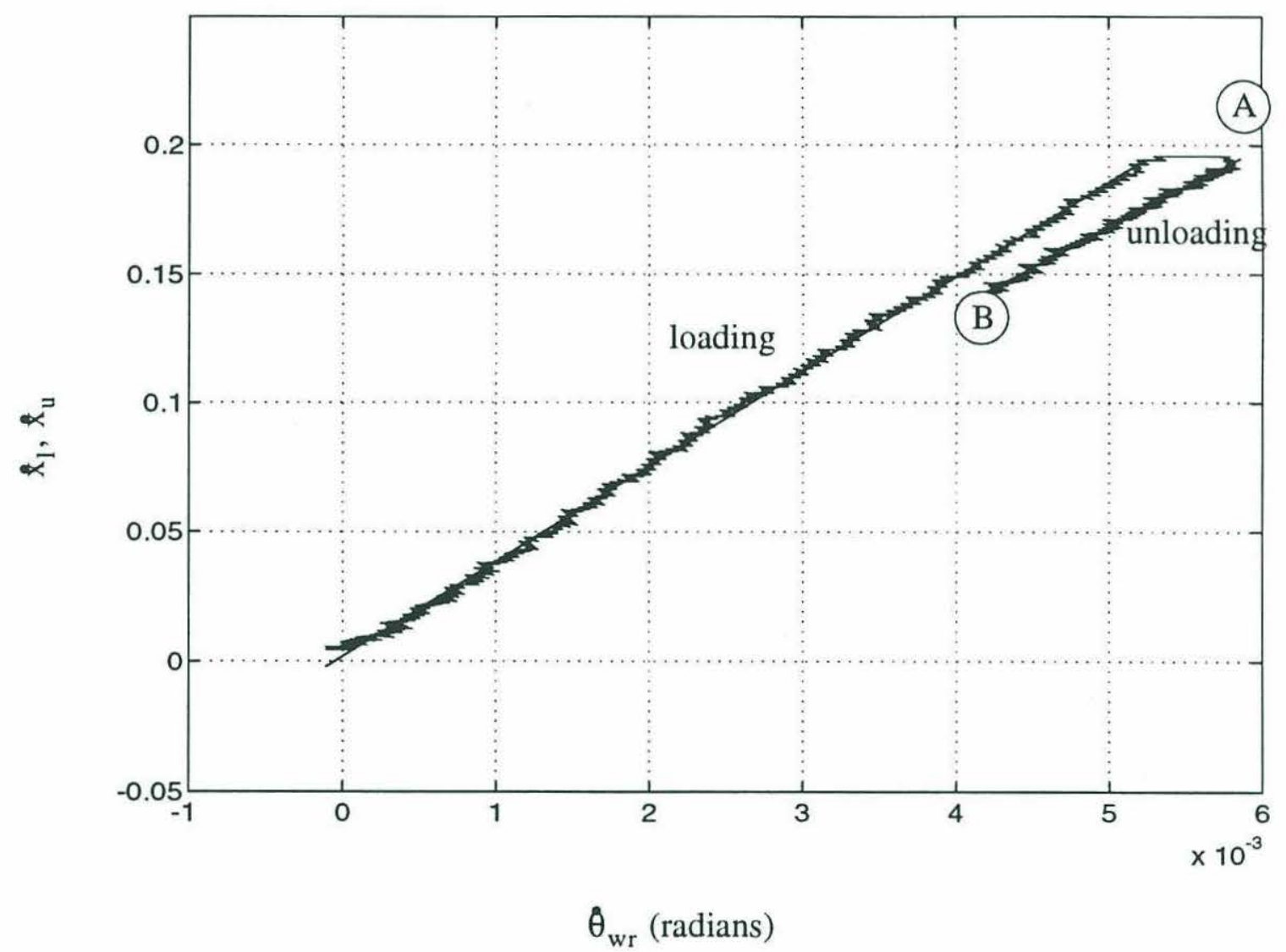

Figure 4.17. $\mathrm{x}_{1}$ and $\mathrm{k}_{\mathrm{u}}$ vs. $\hat{\theta}_{\mathrm{wr}}$, Trial 19

\subsubsection{Correlation Coefficients: All Trials}

The correlation coefficient indicates the how well the model fits the data. Figure 4.18. shows the correlation coefficients associated with the loading (circles) and unloading (x's) models for each of the open circuit trials. This plot indicates that both models fit the data very closely for each trial. The groups of trials that dip noticeably from the rest are trials in 
which we applied and then removed a negative moment (i.e. $\mathrm{M}_{\mathrm{pk}}<0$ ),

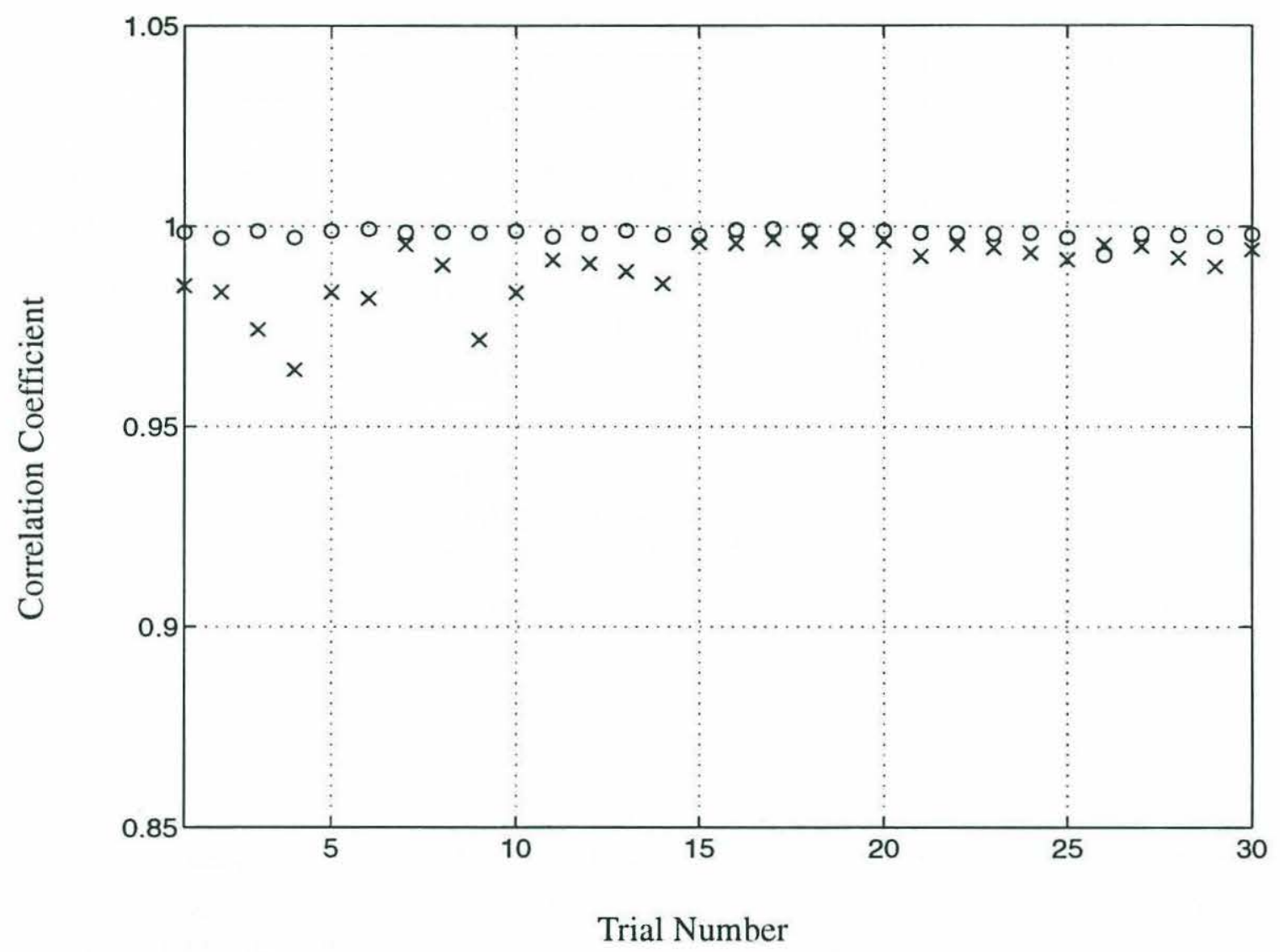

Figure 4.18. Correlation Coefficients, Trials 1 through 30

\subsubsection{Coefficient of Friction: All Trials}

\section{True Coefficient of Friction:}

Referring to Table 4.1 we see that, as with the open circuit trials, there is very good agreement between the average values of the true coefficients of friction obtained from the loading and unloading models. Looking at the actual values from each trial (see upper half of Figure C. 3 in Appendix B) we see that there is excellent agreement between the coefficients of friction obtained from the loading and unloading models in almost every trial.

\section{Acting Coefficient of Friction:}

As was the case with the open circuit experiments in Chapter 3 the acting coefficients of friction do not agree as well as the true coefficients of friction but the shape of the curves is nearly identical, implying that the loading is nearly quasi-static. 


\begin{tabular}{|c|c|c|c|c|c|c|}
\hline \multirow[b]{2}{*}{ Trials } & \multirow[b]{2}{*}{ R (in) } & \multirow[b]{2}{*}{$\mathrm{r}$ (in) } & \multicolumn{2}{|c|}{$\begin{array}{l}\text { True Coefficient of } \\
\text { Friction }\end{array}$} & \multicolumn{2}{|c|}{$\begin{array}{l}\text { Acting Coefficient of } \\
\text { Friction }\end{array}$} \\
\hline & & & $\begin{array}{l}\frac{\text { Loading }}{\text { Mean }} \\
\text { (St. Dev.) } \\
\text { [Uncert.] }\end{array}$ & $\begin{array}{c}\frac{\text { Unloading }}{\text { Mean }} \\
\text { (St. Dev.) } \\
\text { [Uncert.] }\end{array}$ & $\begin{array}{l}\frac{\text { Loading }}{\text { Mean }} \\
\text { (St. Dev.) } \\
\text { [Uncert.] }\end{array}$ & $\begin{array}{c}\frac{\text { Unloading }}{\text { Mean }} \\
\text { (St. Dev.) } \\
\text { [Uncert.] }\end{array}$ \\
\hline $1-25$ & $\begin{array}{c}2.438 " \\
\text { Steel }\end{array}$ & $\begin{array}{c}2.438 " \\
\text { Aluminum }\end{array}$ & $\begin{array}{l}0.094 \\
(0.01) \\
{[.022]}\end{array}$ & $\begin{array}{c}0.10 \\
(0.011) \\
{[.022]}\end{array}$ & $\begin{array}{c}0.099 \\
(0.010) \\
{[-]}\end{array}$ & $\begin{array}{c}0.091 \\
(0.013) \\
{[-]}\end{array}$ \\
\hline $27-30$ & $\begin{array}{c}2.438^{\prime \prime} \\
\text { Steel }\end{array}$ & $\begin{array}{c}2.438 " \\
\text { Aluminum }\end{array}$ & $\begin{array}{c}0.069 \\
(0.004) \\
{[0.009]}\end{array}$ & $\begin{array}{c}0.070 \\
(0.003) \\
{[0.009]}\end{array}$ & $\begin{array}{c}0.069 \\
(0.002) \\
{[-]}\end{array}$ & $\begin{array}{c}0.072 \\
(0.001) \\
{[-]}\end{array}$ \\
\hline
\end{tabular}

Table 4.1 Mean Values of True and Acting Coefficients of Friction

\subsection{Conclusions}

Using the results of Chapter 3 we modelled the deflection of a single stage closed circuit cable drive during loading and unloading. Like the open circuit drive, the geometry-friction number GF determines the character of a closed circuit drive's response to load. We showed that in general we cannot solve explicitly for the equilibrium cable tensions, preventing us from finding a closed form solution for the deflection. However, we identified two special case drives for which we can obtain closed form expressions for the equilibrium tensions; GF $=\infty$ (widely separated pulleys) and GF $=0$ (narrowly separated pulleys). We used these special case solutions to approximate the tensions in drives having intermediate GF values and compared the resulting deflections during loading to numerically obtained results, showing that these approximations give accurate results over a broad range of useful GF values. (see Table 4.2)

\begin{tabular}{|c|c|c|}
\hline GF & $\begin{array}{c}\text { Consider } \\
\text { using }\end{array}$ & $\begin{array}{c}\text { Worst Case } \\
\text { Error } \\
\text { During } \\
\text { Loading }\end{array}$ \\
\hline \hline $0 \leq \mathrm{GF} \approx 1$ & Model III & $\sim 12 \%$ \\
\hline $1 \approx \mathrm{GF} \leq 3$ & Model III & $\sim 18 \%$ \\
\hline
\end{tabular}




\begin{tabular}{|c|c|c|}
\hline GF & $\begin{array}{c}\text { Consider } \\
\text { using }\end{array}$ & $\begin{array}{c}\text { Worst Case } \\
\text { Error } \\
\text { During } \\
\text { Loading }\end{array}$ \\
\hline \hline $3 \approx \mathrm{GF} \leq \infty$ & Model II & $\sim 12 \%$ \\
\hline $10 \approx \mathrm{GF} \leq \infty$ & Model I & $\sim 11 \%$ \\
\hline
\end{tabular}

\section{Table 4.2 Applicable range of approximate models I, II and III}

We ran experiments on one closed circuit drive to test the validity of the GF $=0$ solution showing that it accurately describes the behavior of the drive. In addition, the coefficients of friction obtained from these tests agree with those obtained from open circuit tests performed on the same drive.

\section{Characteristics of Closed Circuit Cable Drives}

Closed circuit drives share most of the characteristics of open circuit drives.

\section{Visco-elastic Deflection Response}

The drives we tested required a significant amount of time to reach their equilibrium deflections. We believe viscous friction forces between the cable and pulley to be responsible for this behavior. If true, the stiffness of cable drives should be higher when the applied load varies rapidly.

\section{Softening Spring behavior}

Cable drives are stiffest near zero load, becoming progressively more compliant as the load increases.

3. Nature of Response Determined Value of $\mathrm{GF}=\frac{\mathrm{L} \mu_{\mathrm{eff}}}{\mathrm{R}}$.

The value of the geometry-friction number GF indicates the relative importance of the linear and nonlinear (softening) components of the drive's stiffness. A high GF value yields a drive with very low but nearly linear stiffness. A low GF value results in a much stiffer drive but this stiffness drops off rapidly as the load increases.

\section{Stiffness Depends on Pretension $\mathrm{T}_{0}$.}




\section{Chapter 4: Closed Circuit Cable/Pulley Drives}

Increasing $\mathrm{T}_{0}$ makes a cable drive stiffer. The effect of a given change in pretension depends on the value of GF.

\section{Inherent hysteresis}

Hysteresis is inherent to cable drives. 


\section{Chapter 5 Multistage Drives}

Size constraints often limit the maximum reduction ratio that can be achieved with a single stage drive. A drive with multiple stages will usually be more compact than an equivalent single stage drive of the same ratio. However, a multistage drive will typically be more complex, have lower stiffness, lower load capacity, and higher friction than its single stage counterpart. Nonetheless, packaging considerations often preclude the use of a single stage design. This chapter extends the theory of Chapters 3 and 4 to multistage versions of open and closed circuit drives. 


\subsection{Multistage Open Circuit Drives}

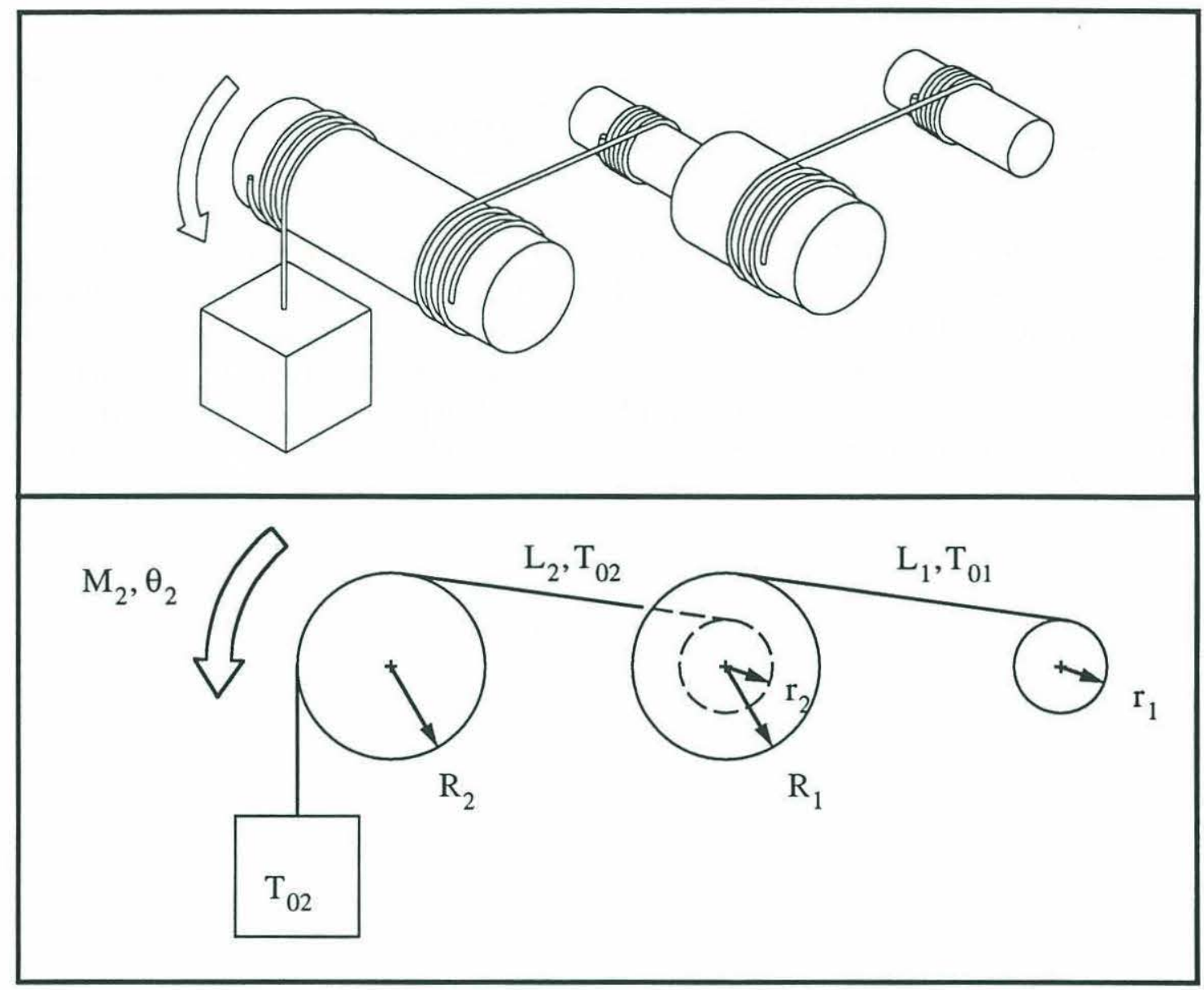

Figure 5.1. Multistage Open Circuit Cable Drive

Figure 5.1. shows a two stage open circuit cable drive. We pretension both stages simultaneously by turning pulley $r_{1}$ through its full range of motion, raising and then lowering the load $\mathrm{T}_{02}$. Stage 2 then has a uniform pretension of $\mathrm{T}_{02}$ while the pretension in the first stage is

$$
\mathrm{T}_{01}=\mathrm{T}_{02} \frac{\mathrm{r}_{2}}{\mathrm{R}_{1}}
$$

Locking the first stage input pulley and applying a moment $\mathrm{M}_{2}$ to the second stage output pulley we wish to determine the relationship between $\mathrm{M}_{2}$ and the resultant deflection $\theta_{2 \text { tot }}$. (For generality we assume that the two stages have different pulley radii, different reduc- 
tion ratios and cables of different stiffness.)

The total deflection of the output pulley will be

$$
\theta_{2 \text { tot }}=\theta_{\text {stage } 1}\left(\frac{r_{2}}{R_{2}}\right)+\theta_{\text {stage 2 }}
$$

where $\theta_{\text {stage } 1}$ and $\theta_{\text {stage } 2}$ are the amounts that the respective output pulleys of each stage would deflect if that stage was independently subjected to the same loads it experiences as part of the multistage drive. Recalling equation (3-16) these deflections are

$$
\begin{aligned}
& \theta_{\text {stage } 1}=\frac{\mathrm{T}_{01}}{\mathrm{EA}_{1}}\left(\frac{1}{\mu_{\text {leff }}}\left(\mathrm{m}_{1}-\log \left(\mathrm{m}_{1}+1\right)\right)+\frac{\mathrm{L}_{1}}{\mathrm{R}_{1}} \mathrm{~m}_{1}\right) \\
& \theta_{\text {stage } 2}=\frac{\mathrm{T}_{02}}{\mathrm{EA}_{2}}\left(\frac{1}{\mu_{2 \text { eff }}}\left(\mathrm{m}_{2}-\log \left(\mathrm{m}_{2}+1\right)\right)+\frac{\mathrm{L}_{2}}{\mathrm{R}_{2}} \mathrm{~m}_{2}\right)
\end{aligned}
$$

where

$$
\begin{aligned}
& \mu_{1 \text { eff }}=\left(\frac{\mathrm{r}_{1}}{\mathrm{R}_{1}} \frac{1}{\mu_{\mathrm{r}_{1}}}+\frac{1}{\mu_{\mathrm{R}_{1}}}\right) \\
& \mu_{2 \text { eff }}=\left(\frac{\mathrm{r}_{1}}{\mathrm{R}_{1}} \frac{1}{\mu_{\mathrm{r}_{2}}}+\frac{1}{\mu_{\mathrm{R}_{2}}}\right)
\end{aligned}
$$

and the dimensionless moments $\mathrm{m}_{1}$ and $\mathrm{m}_{2}$ are

$$
\mathrm{m}_{2}=\frac{\mathrm{M}_{2}}{\mathrm{R}_{2} \mathrm{~T}_{02}}
$$

and

$$
\mathrm{m}_{1}=\frac{\mathrm{M}_{1}}{\mathrm{R}_{1} \mathrm{~T}_{01}}
$$

Using equation (5-1) to eliminate $T_{01}$ and recognizing that $M_{1}=M_{2} \frac{r_{2}}{R_{2}}$ we find that 
$\mathrm{m}_{1}=\mathrm{m}_{2}$, i.e.

$$
\mathrm{m}_{1}=\frac{\mathrm{M}_{1}}{\mathrm{R}_{1} \mathrm{~T}_{01}}=\left(\frac{\mathrm{M}_{2} \frac{\mathrm{r}_{2}}{\mathrm{R}_{2}}}{\mathrm{R}_{1} \mathrm{~T}_{02} \frac{\mathrm{r}_{2}}{\mathrm{R}_{1}}}\right)=\frac{\mathrm{M}_{2}}{\mathrm{R}_{2} \mathrm{~T}_{02}}=\mathrm{m}_{2}
$$

Thus we can rewrite equation (5-3) as

$$
\theta_{\text {stage } 1}=\frac{\mathrm{r}_{2}}{\mathrm{R}_{1}} \frac{\mathrm{T}_{02}}{\mathrm{EA}_{1}}\left(\frac{1}{\mu_{\text {leff }}}\left(\mathrm{m}_{2}-\log \left(\mathrm{m}_{2}+1\right)\right)+\frac{\mathrm{L}_{1}}{\mathrm{R}_{1}} \mathrm{~m}_{2}\right)
$$

and equation (5-2) becomes

$$
\theta_{2 \text { tot }}=\frac{\mathrm{T}_{02}}{\mathrm{EA}_{2}}\left[\left[\frac{\mathrm{r}_{2}^{2}}{\mathrm{R}_{1} \mathrm{R}_{2}} \frac{\mathrm{EA}_{2}}{\mathrm{EA}_{1}} \frac{1}{\mu_{\text {leff }}}+\frac{1}{\mu_{2 \text { eff }}}\right]\left(\mathrm{m}_{2}-\log \left(\mathrm{m}_{2}+1\right)\right)+\left[\left(\frac{\mathrm{r}_{2}}{\mathrm{R}_{1}}\right)^{2} \frac{\mathrm{EA}_{2}}{\mathrm{EA}_{1}} \frac{\mathrm{L}_{1}}{\mathrm{R}_{2}}+\frac{\mathrm{L}_{2}}{\mathrm{R}_{2}}\right] \mathrm{m}_{2}\right](5-1
$$

By rearranging terms we can rewrite this as

$$
\theta_{2 \text { tot }}=\frac{\mathrm{T}_{02}}{\mathrm{EA}_{2} \mu_{\mathrm{ms} 2}}\left[\left(1+\mathrm{GF}_{\mathrm{ms} 2}\right) \mathrm{m}_{2}-\log \left(\mathrm{m}_{2}+1\right)\right]
$$

where

$$
\begin{gathered}
\mathrm{GF}_{\mathrm{ms} 2}=\frac{\mathrm{L}_{\mathrm{ms} 2} \mu_{\mathrm{ms} 2}}{\mathrm{R}_{2}} \\
\frac{1}{\mu_{\mathrm{ms} 2}}=\left[\left(\frac{\mathrm{r}_{2}}{\mathrm{R}_{2}}\right)^{2} \frac{\mathrm{R}_{2}}{\mathrm{R}_{1}} \frac{\mathrm{EA}_{2}}{\mathrm{EA}_{1}}\right] \frac{1}{\mu_{1 \mathrm{eff}}}+\frac{1}{\mu_{2 \mathrm{eff}}} \\
\mathrm{L}_{\mathrm{ms} 2}=\left(\frac{\mathrm{r}_{2}}{\mathrm{R}_{2}}\right)^{2}\left(\frac{\mathrm{R}_{2}}{\mathrm{R}_{1}}\right)^{2} \frac{\mathrm{EA}_{2}}{\mathrm{EA}_{1} \mathrm{~L}_{1}+\mathrm{L}_{2}}
\end{gathered}
$$

By comparing equation (5-15) with equation (3-16) we see that the behavior of a two stage open circuit drive is identical to that of an equivalent single stage drive where the effective values of GF, $\mathrm{L}$ and $\mu_{\mathrm{eff}}$ are given by equations (5-13) through (5-15). For a three stage drive these constants are 


$$
\begin{gathered}
\mathrm{GF}_{\mathrm{ms} 3}=\frac{\mathrm{L}_{\mathrm{ms} 3} \mu_{\mathrm{ms} 3}}{\mathrm{R}_{3}} \\
\frac{1}{\mu_{\mathrm{ms} 3}}=\left[\left(\frac{\mathrm{r}_{2} \mathrm{r}_{3}}{\mathrm{R}_{2} \mathrm{R}_{3}}\right)^{2} \frac{\mathrm{R}_{3}}{\mathrm{R}_{1}} \frac{\mathrm{EA}_{3}}{\mathrm{EA}}\right] \frac{1}{\mu_{1 \mathrm{eff}}}+\left[\left(\frac{\mathrm{r}_{3}}{\mathrm{R}_{3}}\right)^{2} \frac{\mathrm{R}_{3}}{\mathrm{R}_{2}} \frac{\mathrm{EA}_{3}}{\mathrm{EA}_{2}}\right] \frac{1}{\mu_{2 \mathrm{eff}}}+\frac{1}{\mu_{3 \mathrm{eff}}} \\
\mathrm{L}_{\mathrm{ms} 3}=\left[\left(\frac{\mathrm{r}_{2} \mathrm{r}_{3}}{\mathrm{R}_{2} \mathrm{R}_{3}}\right)^{2}\left(\frac{\mathrm{R}_{3}}{\mathrm{R}_{1}}\right)^{2} \frac{\mathrm{EA}_{3}}{\mathrm{EA}_{1}}\right] \mathrm{L}_{1}+\left[\left(\frac{\mathrm{r}_{3}}{\mathrm{R}_{3}}\right)^{2}\left(\frac{\mathrm{R}_{3}}{\mathrm{R}_{2}}\right)^{2} \frac{\mathrm{EA}_{3}}{\mathrm{EA}_{2}}\right] \mathrm{L}_{2}+\mathrm{L}_{3}
\end{gathered}
$$

In the general case, if $k$ is the number of stages in the drive, these values become

$$
\begin{gathered}
\mathrm{GF}_{\mathrm{msk}}=\frac{\mathrm{L}_{\mathrm{msk}} \mu_{\mathrm{msk}}}{\mathrm{R}_{\mathrm{k}}} \\
\frac{1}{\mu_{\mathrm{msk}}}=\sum_{\mathrm{i}=1}^{\mathrm{k}}\left[\left(\mathrm{N}_{\mathrm{ik}}\right)^{2} \frac{\mathrm{R}_{\mathrm{k}}}{\mathrm{R}_{\mathrm{i}}} \frac{\mathrm{EA}_{\mathrm{k}}}{\mathrm{EA}_{\mathrm{i}}}\right]\left(\frac{1}{\mu_{\text {eff }}}\right)_{\mathrm{i}} \\
\mathrm{L}_{\mathrm{msk}}=\sum_{\mathrm{i}=1}^{\mathrm{k}}\left[\left(\mathrm{N}_{\mathrm{ik}}\right)^{2}\left(\frac{\mathrm{R}_{\mathrm{k}}}{\mathrm{R}_{\mathrm{i}}}\right)^{2} \frac{\mathrm{EA}_{\mathrm{k}}}{\mathrm{EA}_{\mathrm{i}}}\right] \mathrm{L}_{\mathrm{i}}
\end{gathered}
$$

where $\mathrm{N}_{\mathrm{ik}}$ is the reduction ratio between the output axis of stage $i$ and the output axis of stage $k$, or

$$
N_{i k}=\left[\begin{array}{cc}
\prod_{j=i}^{k} \frac{r_{j+1}}{R_{j+1}} & i \neq k \\
1 & i=k
\end{array}\right]
$$

(Note: In this convention the input pulley is part of the first stage and the output pulley is part of the $k$ th stage).

A similar analysis yields the hysteresis deflection for a multi-stage open circuit drive. We look at the case where the two stage drive shown in Figure 5.1. has been loaded to some peak torque $M_{2 \text { peak }}$ which has subsequently been decreased to the current load $M_{2}$. The total hysteresis deflection at the output pulley of the last stage is 


$$
\theta_{\mathrm{h}}=\frac{\mathrm{r}_{2}}{\mathrm{R}_{2}} \theta_{\text {hstage } 1}+\theta_{\text {hstage } 2}
$$

where $\theta_{\text {hstage } 1}$ and $\theta_{\text {hstage2 }}$ are the hysteresis deflections that the respective output pulleys of each stage would experience when that stage was independently subjected to the same loads it experiences as part of the multistage drive. These deflections are

$$
\begin{aligned}
& \theta_{1 \text { hstage }}=\frac{\mathrm{T}_{01}}{\mathrm{EA}_{1} \mu_{1 \text { eff }}}\left[\sqrt{\left(\mathrm{m}_{1 \text { peak }}+1\right)\left(\mathrm{m}_{1}+1\right)}-\mathrm{m}_{1}-2-\ln \left(\mathrm{m}_{1 \text { peak }}+1\right)\right]+\frac{\mathrm{T}_{01} \mathrm{~L}_{1}}{\mathrm{EA}_{1} \mathrm{R}_{1}} \mathrm{~m}_{1} \\
& \theta_{2 \text { bstage }}=\frac{\mathrm{T}_{02}}{\mathrm{EA}_{2} \mu_{2 \text { eff }}}\left[\sqrt{\left(\mathrm{m}_{\text {peak } 2}+1\right)\left(\mathrm{m}_{2}+1\right)}-\mathrm{m}_{2}-2-\ln \left(\mathrm{m}_{2 \text { peak }}+1\right)\right]+\frac{\mathrm{T}_{02} \mathrm{~L}_{2}}{\mathrm{EA}_{2} \mathrm{R}_{2}} \mathrm{~m}_{2}
\end{aligned}
$$

Using equations (5-1) and (5-9) to substitute for $\mathrm{T}_{01}$ and $\mathrm{m}_{1}$ in equation (5-23) we get

$$
\theta_{\mathrm{h}}=\frac{\mathrm{T}_{2}}{\mathrm{EA}_{2} \mu_{\mathrm{ms} 2}}\left[\sqrt{\left(\mathrm{m}_{\text {peak } 2}+1\right)\left(\mathrm{m}_{2}+1\right)}-\left(1-\mathrm{GF}_{\mathrm{ms} 2}\right) \mathrm{m}_{2}-2-\ln \left(\mathrm{m}_{2 \text { peak }}+1\right)\right]
$$

where $\mathrm{GF}_{\mathrm{ms} 2}$ and $\mu_{\mathrm{ms} 2}$ are the same as above. We see that the hysteresis deflection of a multistage drive can also be modeled as that of an equivalent single stage drive, the same equivalent drive as was found before. To obtain the hysteresis model for an arbitrary drive with $k$ stages, we substitute $\mathrm{GF}_{\mathrm{msk}}$ and $\mu_{\mathrm{msk}}$ (equations (5-19) and (5-20)) for $\mathrm{GF}_{\mathrm{ms} 2}$ and $\mu_{\mathrm{ms} 2}$.

\subsection{Multistage Closed Circuit Drives}

There are two types of multistage closed circuit drives. The first is made up of independently pretensioned stages, i.e. the drive is made up of $k$ individual closed circuit drives connected in series. The second type is made up of two multistage open circuit drives linked antagonistically by their input pulleys at one end and by their output pulleys at the other, i.e. there is one closed circuit, each half of which is a $k$ stage open circuit drive.We will investigate both possibilities and will discuss the design implications of each approach. 


\subsubsection{Two Antagonistic Multistage Open Circuit Drives in Parallel}

In Chapter 4 we analyzed the single stage closed circuit drive as being made from two antagonistic single stage open circuit drives in parallel. We showed in the previous section that a multistage open circuit drive behaves exactly like an equivalent single stage drive. Therefore a closed circuit drive made from multistage open circuit drives will behave exactly like an equivalent single stage closed circuit drive. The equations and curves presented in Chapter 4 all apply if $\mathrm{GF}_{\mathrm{msk}}, \mu_{\mathrm{msk}}$, and $\mathrm{L}_{\mathrm{msk}}$ from section 5.1 are substituted for the values GF, $\mu_{\mathrm{eff}}$, and $\mathrm{L}$ in Chapter 4 .

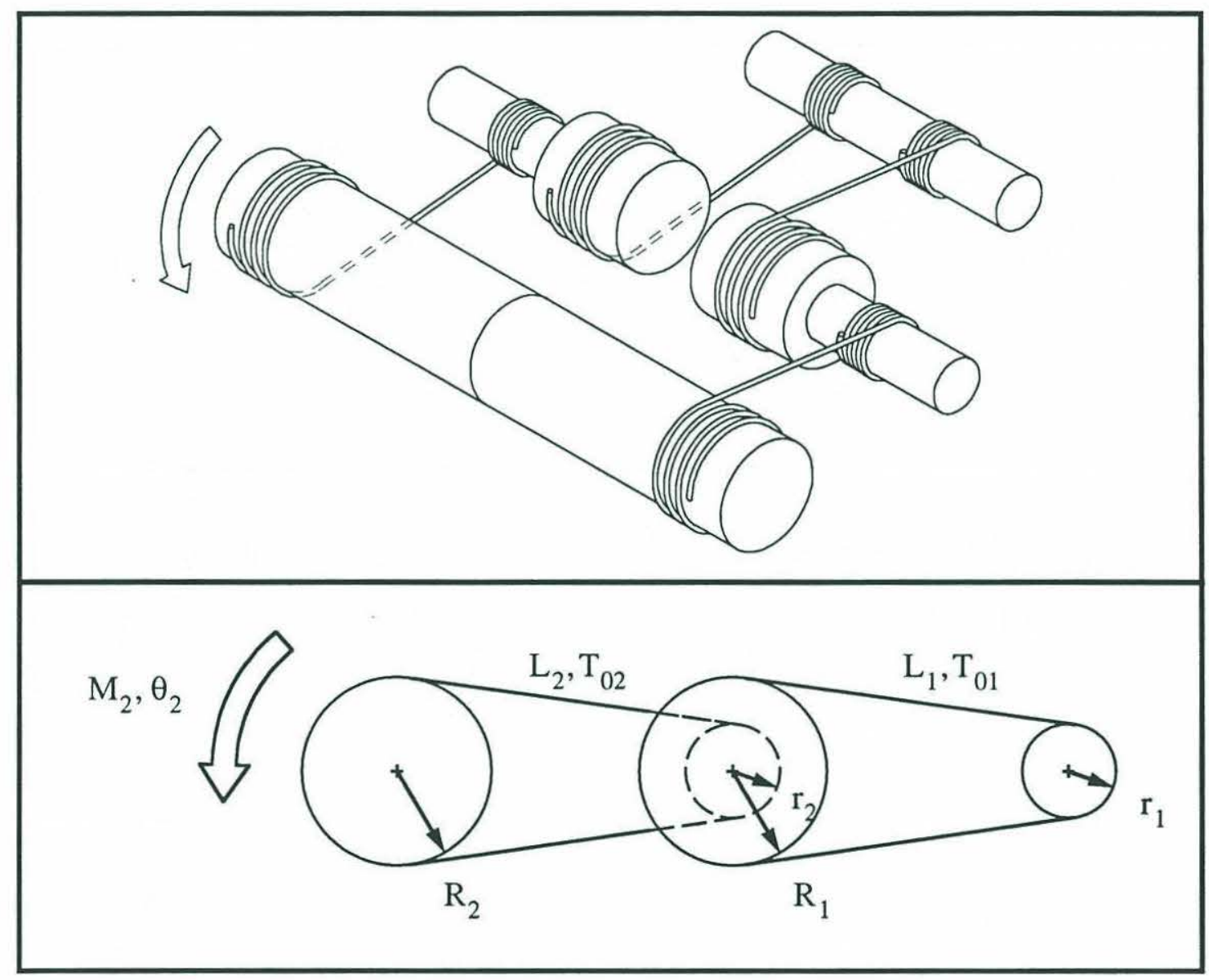

Figure 5.2 Multistage Closed Circuit Drive Made from Two Multistage Open Circuit Drives 


\subsubsection{Individual Closed Circuit Drives in Series}

This type of drive is more difficult to analyze. We cannot characterize it by a single GF value as we could above. This system has $k$ independent closed circuit stages, each with its own pretension, GF, and $\mu_{\text {eff }}$. Each of these circuits must be analyzed independently, requiring that we either numerically solve equation (4-15) $k$ times (or use the curves in Chapter $4 k$ times) to determine the behavior of each stage and then combine the effects of these stages to determine the behavior of the aggregate drive. While this amount of effort may be worthwhile for a final design, it is a bit cumbersome for the conceptual and embodiment phases of a design. We therefore look for a way to simplify the analysis.

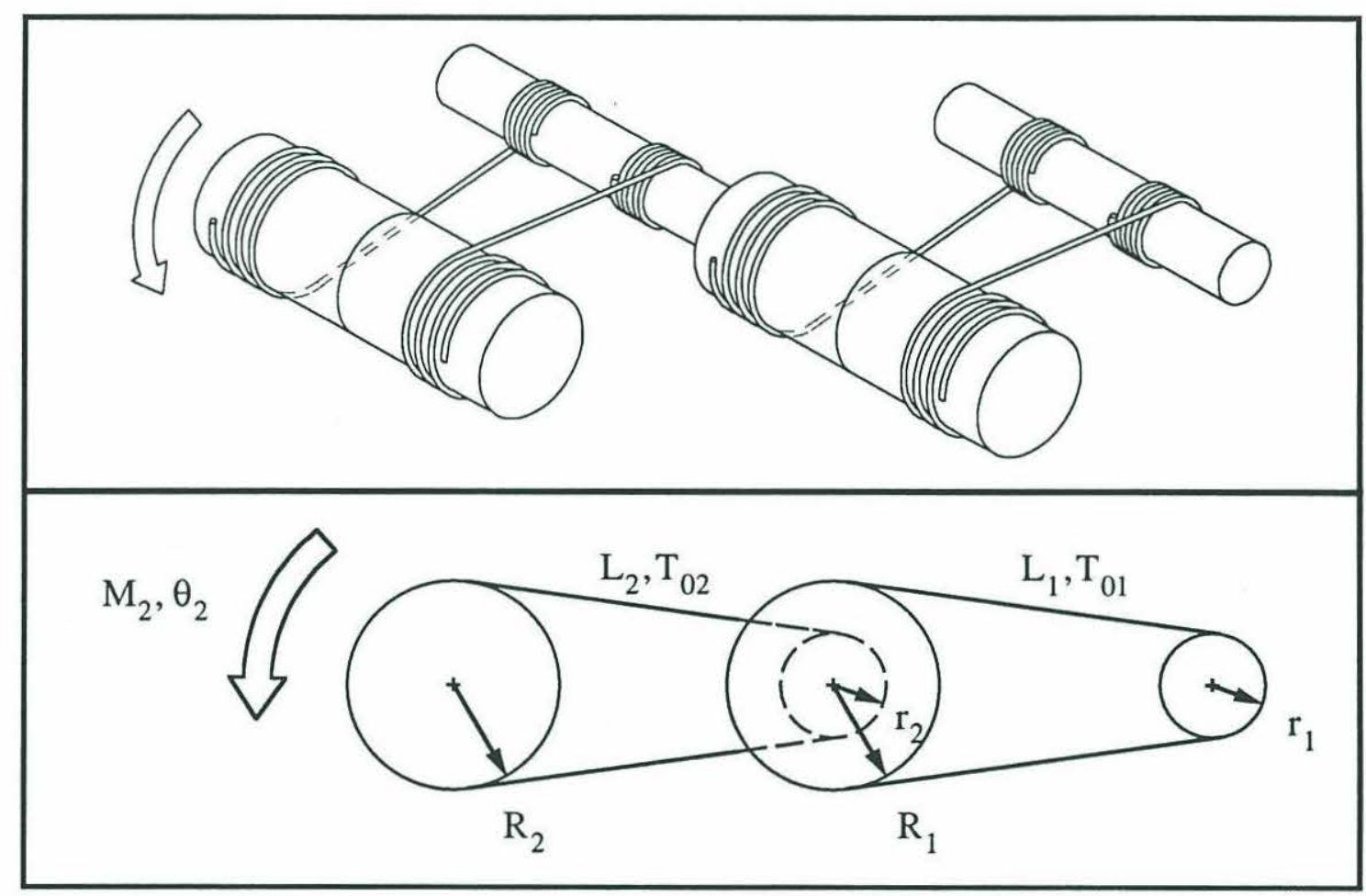

Figure 5.3 Multistage Closed Circuit Drive Made from Cascaded Single Stage Closed Circuit Drives

The deflection of a drive made up of multiple single stage closed circuit drives connected in series is

$$
\theta_{k}(M)=\sum_{i=1}^{k} N_{i k} \theta_{\text {stagei }}(M)
$$

where $k$ is the number of single stage circuits, $\mathrm{N}_{\mathrm{ik}}$ is the reduction ratio between the output 
Multistage Closed Circuit Drives:

axes of stage $i$ and stage $k$ and $\mathrm{M}$ is the moment applied to the output pulley of the $k$ th stage. $\theta_{\text {stagei }}(M)$ is the deflection that the output pulley of stage $i$ would experience if it was independently subjected to the same loads it experiences as part of the multiple stage drive and is equal to

$$
\theta_{\text {stagei }}(M)=\frac{T_{0 i}}{\mathrm{EA}_{\mathrm{i}} \mu_{\text {effi }}}\left[\left(1+\mathrm{GF}_{\mathrm{i}}\right)\left(\tau_{\mathrm{li}}(\mathrm{M})-1\right)-\log \left(\tau_{\mathrm{li}}(\mathrm{M})\right)\right]
$$

The ideal way to simplify equation (5-27) is configure the drive such that all of terms $\theta_{\text {stagei }}(M)$ are of the same form, i.e. that the term in the square brackets in (5-28) is the same for every stage. The total deflection will then be some constant times this one term. To achieve this we need to make sure that every stage experiences the same dimensionless tension $\tau_{1}(\mathrm{M})$ and the same GF value.

There are two ways to make all the stages have the same GF value. One is to make every stage identical, having the same pulley sizes, tangent lengths, and coefficients of friction. This has the benefit of using identical parts throughout the drive, simplifying the design, fabrication, and maintenance of the finished drive. The other option is to make the GF for each of the circuits so small as to be negligible (say, GF less than about.03) so that we can make the approximation GF $=0$ for all the stages. Note that the individual GF values don't have to be equal, just small. This approach has the benefits of maximizing the drive stiffness and results in a closed form solution for the nondimensional tensions and the drive deflection.

To make all of the dimensionless tensions for the $k$ stages equal we look back to equation (4-15), the equilibrium equation for a single stage closed circuit drive under load.

$$
\log \left(\frac{\tau_{1}}{\tau_{1}+\mathrm{m}}\right)+(1+\mathrm{GF}) \mathrm{m}+\mathrm{GF}\left(2 \tau_{1}-2\right)=0
$$

We see that the value of GF determines the relationship between the applied moment and the resulting cable tensions. Two stages will have the same relationship between $\mathrm{m}$ and $\tau_{1}$ if they have the same value of GF but they won't have the same value of $\tau_{1}$ unless the dimensionless moments are also the same.

When the moment $\mathrm{M}_{\mathrm{k}}$ is applied to the output axis of the final stage $k$ the moment acting on an intermediate stage $i$ is 


$$
M_{i}=\left(N_{i k}\right) M_{k}
$$

where $\mathrm{N}_{\mathrm{ik}}$ is the reduction ratio between the output axis of stage $i$ and the output axis of the final stage $k$. The dimensionless moment for stage $i$ is

$$
m_{i}=\frac{M_{i}}{R_{i} T_{0 i}}=N_{i k} \frac{M_{k}}{R_{i} T_{0 i}}
$$

while the dimensionless moment for stage $k$ is

$$
\mathrm{m}_{\mathrm{k}}=\frac{\mathrm{M}_{\mathrm{k}}}{\mathrm{R}_{\mathrm{k}} \mathrm{T}_{0 \mathrm{k}}}
$$

From these two equations we see that the nondimensional moment will be constant from stage to stage when

$$
\mathrm{T}_{0 \mathrm{i}}=\mathrm{T}_{0 \mathrm{k}}\left(\mathrm{N}_{\mathrm{ik}} \frac{\mathrm{R}_{\mathrm{k}}}{\mathrm{R}_{\mathrm{i}}}\right)
$$

These pretensions are identical to those that occur naturally in a multistage open circuit drive. With these pretensions and a common value of GF between stages the behavior of the entire multistage drive will be described by a single solution of equation (4-15). Equation (5-27) can now be rewritten as

$$
\theta_{k}(M)=\left(\sum_{i=1}^{k} N_{i k} \frac{T_{0 i}}{E_{i} \mu_{e f f i}}\right)\left[(1+G F)\left(\tau_{1}(M)-1\right)-\log \left(\tau_{1}(M)\right)\right]
$$

or

$$
\theta_{\mathrm{k}}(\mathrm{M})=\left(\sum_{\mathrm{i}=1}^{\mathrm{k}}\left(\mathrm{N}_{\mathrm{ik}}\right)^{2} \frac{\mathrm{T}_{0 \mathrm{k}}}{\mathrm{EA}_{\mathrm{i}} \mu_{\mathrm{effi}}}\right)\left[(1+\mathrm{GF})\left(\tau_{1}(\mathrm{M})-1\right)-\log \left(\tau_{\mathrm{l}}(\mathrm{M})\right)\right.
$$

The summation term is now made up entirely of constants and becomes just a scale factor for the term in square brackets. If $\mathrm{GF} \approx 0$ we can solve explicitly for $\tau_{1}$ and equation (535) becomes 


$$
\theta_{k}(M)=\left(\sum_{i=1}^{k}\left(N_{i k}\right)^{2} \frac{T_{0 k}}{E A_{i} \mu_{e f f i}}\right)\left[(1+G F)\left(\frac{m_{k}}{e^{m_{k}}-1}-1\right)-\log \left(\frac{m_{k}{ }^{(5-36)}}{e^{m_{k}}-1}\right)\right]
$$

where

$$
\mathrm{m}_{\mathrm{k}}=\frac{\mathrm{M}_{\mathrm{k}}}{\mathrm{R}_{\mathrm{k}} \mathrm{T}_{0 \mathrm{k}}}
$$

\subsection{Conclusions}

We have identified several important characteristics of multistage cable drives.

\section{Multistage Open Circuit Drives:}

1. Multistage open circuit drives can be modelled as equivalent single stage open circuit drives where the effective values of $G F, L$ and $\mu_{\text {eff }}$ are given by

- equations (5-13) through (5-15) (for two stage drives)

- equations (5-16) through (5-18) (for three stage drives)

- equations (5-19) through (5-21) (for drives with $k$ stages)

\section{Multistage Closed Circuit Drives:}

There are two types of multistage closed circuit drives.

1. Multistage drives made from opposing multistage open circuit drives - can be modelled as equivalent single stage closed circuit drives where the effective values for $G F, L$ and $\mu_{\text {eff }}$ are the same as given above.

2. Multistage drives made from $k$ single stage closed circuit drives in series - each stage must be modelled as a separate single stage closed circuit drive. Only under special circumstances can the analysis of the drive be simplified (see section 5.2.2 for details). 


\section{Chapter 6 Designing Cable Drives}

We now discuss how to use the results of the previous chapters in the design of practical cable drives for real applications. We start by summarizing the properties of cable drives and by identifying the application areas (i.e. high performance force and position control) which benefit most from this combination of properties (i.e. zero backlash, low stiction and high stiffness). Following this we summarize the appropriate expressions for the GF number for a variety of drive configurations and highlight over what range of GF values each of the approximate models from Chapter 4 applies. After a brief overview of the relationships between the design parameters and the drive's physical characteristics the chapter ends by identifying the limitations of the cable drive models. 


\section{Chapter 6: Designing Cable Drives}

\subsection{Suitable Applications}

Cable drives should be considered for any application requiring high resolution, high bandwidth control of a position or applied force. In these applications the physical characteristics of the drive mechanism (specifically stiction forces, compliance and backlash) typically limit the performance of the overall system. As with a number of other transmission mechanisms, cable drives avoid backlash by means of a preload, ensuring the existence of a closed force path between the input and output at all times. Unfortunately preloading a transmission mechanism also increases the friction and stiction forces present in the drive. To date cable drives appear to incur a lower friction penalty than any other zero backlash mechanism (Schempf[11]). In addition this same work showed that cable drives can have stiffness values comparable to that of the competing zero backlash transmission options. Thus a cable drive is an excellent choice for high performance force and position control applications.

\begin{tabular}{|c|c|}
\hline Advantages & Disadvantages \\
\hline \hline Zero Backlash & Limited Range of Motion \\
Low Stiction/Friction & Not Off-the-Shelf \\
High Stiffness & Relative Size \\
Low Torque Ripple & Relative Complexity \\
\hline
\end{tabular}

Table 6.1 Advantages and Disadvantages of Cable Pulley Drives

The designer should be aware of two other traits of cable drives.

\section{Softening Spring Behavior}

The stiffness of most cable drives decreases significantly as the applied load increases, having maximum stiffness at low loads. In contrast, most other zero backlash transmission mechanisms exhibit stiffening spring behavior, having very low stiffness for low loads and higher stiffness at higher loads. In addition the stiffness of cable drives is a quantifiable function of the design parameters, enabling better prediction of the performance of the actual system.

\section{Hysteresis Deflection}

In most other zero backlash transmissions hysteresis is due primarily to friction/stiction forces present in the drive and is therefore difficult to predict. Hysteresis in cable drives is a quantifiable function of the design parameters, allowing the designer to understand the impact of various design choices on the expected hysteresis deflection for given drive. 
Table 6.2 $\mu_{\text {eff }}, \mathrm{L}_{\text {eff }}$ and $R$ for Closed Circuit Drives Composed of Opposing Open Circuit Drives

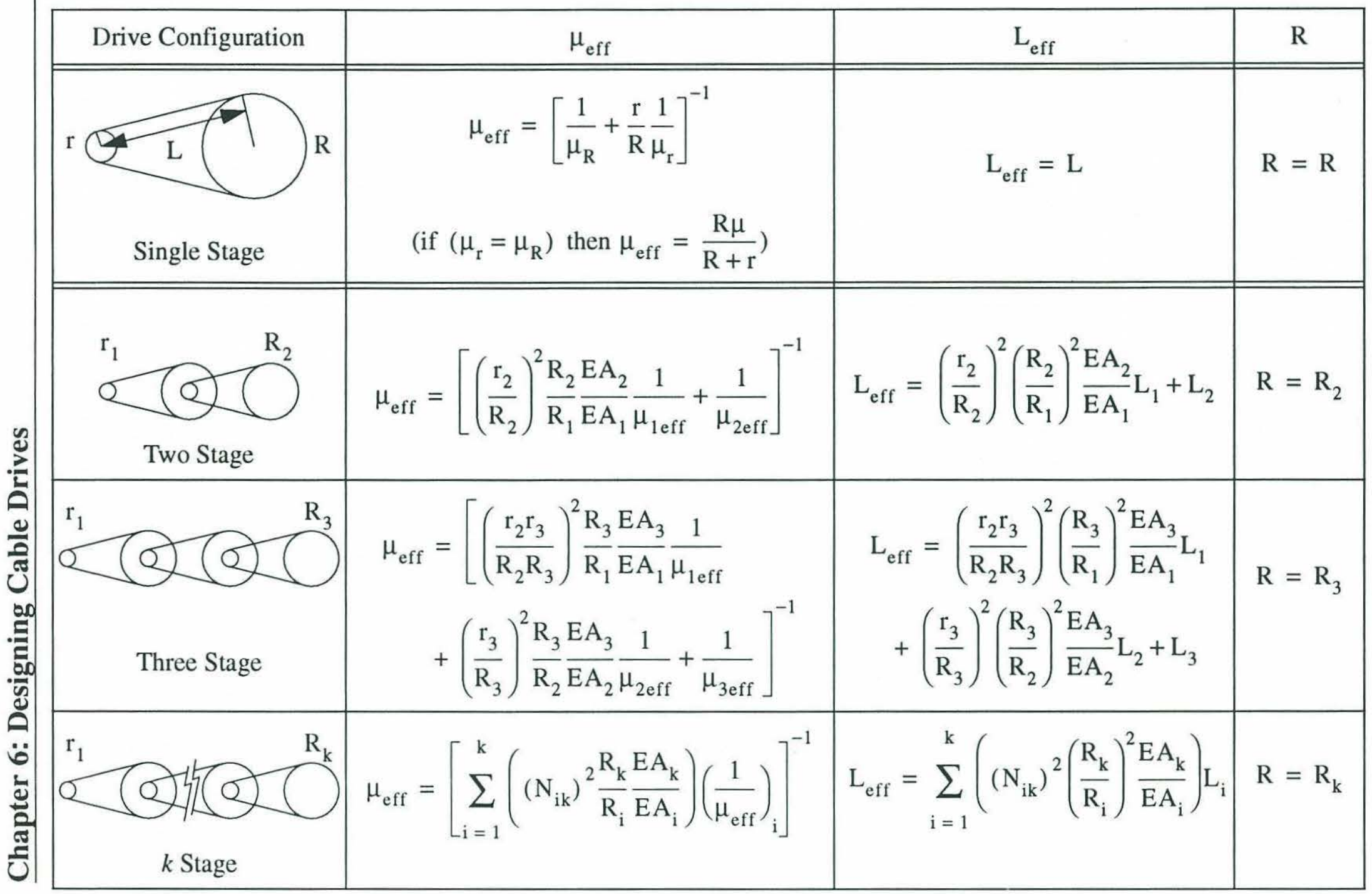




\section{Chapter 6: Designing Cable Drives}

\subsection{Choosing the Appropriate Model for a Given Cable Drive}

In Chapters 4 and 5 we found that the behavior of all single stage and many multistage closed circuit drives is determined by the value of the Geometry-Friction number GF, a dimensionless parameter which indicates the relative elongation of the unwrapped and wrapped sections of cable in the drive. When GF » 1 the unwrapped cable accounts for most of the drive's deflection and the drive has a very low but nearly linear stiffness. When $\mathrm{GF} \ll 1$ the wrapped cable dominates and the drive has a much higher but nonlinear stiffness which decreases markedly as the applied load increases. Thus we use the value of GF to determine the appropriate approximate model for a particular drive.

We define GF as

$$
\mathrm{GF} \equiv \frac{\mathrm{L}_{\text {eff }} \mu_{\text {eff }}}{\mathrm{R}}
$$

where $\mathrm{R}, \mathrm{L}_{\mathrm{eff}}$ and $\mu_{\mathrm{eff}}$ are, respectively, the radius of the drive's output pulley, the effective tangent length and the effective coefficient of friction. The appropriate values of $L_{\text {eff }}, \mu_{\text {eff }}$ and $\mathrm{R}$ are given in Table 6.2 for several possible drive configurations. Once the GF value for a drive is known use Table 6.3 to determine the appropriate approximate model to use, where Models I, II, and III are those presented in Section 4.2.6. In reality, almost all practical cable drives fall into the GF $<3$ category, meaning that Model III should be used to describe most drives. Recalling the structure of these models we see that the lower the GF value the more complex the model required to accurately describe the behavior of the drive.

\begin{tabular}{|c|c|c|}
\hline GF & $\begin{array}{c}\text { Consider } \\
\text { using }\end{array}$ & $\begin{array}{c}\text { Worst Case } \\
\text { Deflection Error } \\
\text { During Loading }\end{array}$ \\
\hline \hline $0 \leq \mathrm{GF} \approx 1$ & Model III & $\sim 12 \%$ \\
\hline $1 \approx \mathrm{GF} \leq 3$ & Model III & $\sim 18 \%$ \\
\hline $3 \approx \mathrm{GF} \leq \infty$ & Model II & $\sim 12 \%$ \\
\hline $10 \approx \mathrm{GF} \leq \infty$ & Model I & $\sim 11 \%$ \\
\hline
\end{tabular}

\section{Table 6.3 Applicable range of approximate models I, II and III}

NOTE: Strictly speaking the information given in Table 6.2 on multistage drives is only valid for multistage drives which are made from opposing multistage open circuit drives (see Section 5.2.1). To obtain accurate results for multistage drives consisting of a number of single stage closed circuit drives connected in series each stage must be modelled sepa- 
rately (see Section 5.2.2). However, the results obtained by modelling a series type drive as an opposing multistage open circuit drive should be conservative, i.e. the actual deflection should be lower than the predicted deflection.

\subsection{Performance Characteristics and Design Parameters}

\begin{tabular}{|l|l|}
\hline Performance Characteristics & Design Parameters \\
\hline \hline Range of Motion & Pretension \\
Reduction Ratio & Number of Stages \\
Physical Size & Pulley Radii \\
Stiffness & Pulley Widths \\
Bandwidth & Pulley Surface Features \\
Positioning Accuracy & Center-Center Distances \\
Fatigue Life & Cable Rigging \\
Load Capacity & Cable Lengths \\
Efficiency & Cable Diameter \\
& Cable Construction \\
& Cable Coating \\
& \\
\hline
\end{tabular}

Table 6.4 Cable Drive Design Parameters and Drive Characteristics

In order to design a successful drive we must understand how the design parameters (e.g. physical dimensions, choice of materials, etc.) affect the desired physical characteristics (e.g. stiffness, efficiency etc.) of the drive. Table 6.4 lists the major performance characteristics and the design parameters which affect them. We briefly discuss the general relations of the various characteristics and design parameters below. For a given drive the models permit the designer to easily determine, among other things, the

- maximum expected hysteresis deflection (i.e. maximum open loop position error)

- deflection and stiffness at various loads levels

- maximum cable tension (and therefore maximum cable stress which necessary to determine the load capacity and fatigue life of drive).

- trade-off between pretension, stiffness and fatigue life 


\section{Chapter 6: Designing Cable Drives}

\subsubsection{Performance Characteristics}

\section{Reduction Ratio}

The reduction ratio depends only on the pulley radii and to a lesser extent on the cable diameter. The models presented assume that $\mathrm{d}$ « $\mathrm{r}, \mathrm{R}$ which will be true for properly designed drives using steel cables (see Load Capacity/Fatigue Life section below). To ensure the accuracy of the predicted reduction ratios, however, we recommend the use of the effective pulley radii in the models. These effective radii are defined as $R=R_{p}+\frac{d}{2}$ and $r=r_{p}+\frac{d}{2}$ where $R_{p}$ and $r_{p}$ are the actual radii of the pulleys and $d$ is the diameter of the cable (thus $\mathrm{r}$ and $\mathrm{R}$ are the distances from the pulley axis to the line of action of the net cable force). The reduction ratio for a drive with $k$ stages is then

$$
\mathrm{N}=\prod_{1}^{\mathrm{k}} \frac{\mathrm{r}_{\mathrm{i}}}{\mathrm{R}_{\mathrm{i}}}
$$

\section{Physical Size}

The size of the drive depends primarily on the center to center distance for each stage and the pulley radii used in the first and last stages. If the drive has only one stage or the multiple stages of the drive are laid out in a line the length of the drive will be

$$
l=\sum_{i=1}^{k} \mathrm{CC}_{i}+r_{1}+R_{k} .
$$

\section{Stiffness/Deflection}

The stiffness of a cable drive depends on the physical parameters $r_{i}, R_{i}, L_{i}, E A_{i}$ and $\mu_{\text {eff }}$, the pretension(s) $\mathrm{T}_{0_{\mathrm{i}}}$ and the applied load $\mathrm{M}$. Increasing any of the $\mathrm{EA}_{\mathrm{i}}, \mathrm{R}_{\mathrm{i}}, \mathrm{T}_{0_{\mathrm{i}}}$ or $\mu_{\text {eff }_{\mathrm{i}}}$ increases the stiffness of the drive while increases in $r_{i}, L_{i}$ and $M$ decrease the stiffness.

In addition, the stiffness also depends on time history of the applied load. For instance, if the current load is the first load applied since the drive was pretensioned (or if it is the first load applied after a large no-load motion of the transmission) the loading model of the deflection should be used. For subsequent loads or cyclic loading the unloading model determines the stiffness and deflection of the drive (actually, as shown in Figure 3.4. the deflection will follow a different version of the unloading equation every time the rate of change of the applied load changes sign). 


\section{Bandwidth}

The models presented in this report describe the behavior of cable drives in static equilibrium. While the experimental data presented in this report confirm the validity of these models they also indicate that viscous (i.e. velocity dependent) effects play a very important role in non-equilibrium situations. However, we expect that the behavior will be qualitatively the same and can say without hesitation that dynamic stiffness will always equal or exceed the static stiffness. Therefore using the static equations to estimate the drive's bandwidth should give a conservative estimate. However, since the drive's stiffness is nonlinear and dependent on the load history there is no simple way to calculate the bandwidth other than through nonlinear simulation.

\section{Positioning Accuracy}

The worst case open loop positioning error for the drive would be the hysteresis deflection predicted by the unloading model for the case when the maximum expected load has been applied to and then removed from the drive. If the position loop is closed around the transmission the hysteresis deflection itself should have no discernible impact on the overall positioning performance of a system. However, the internal drive dynamics may dominate the control problem.

\section{Load Capacity and Fatigue Life}

The typical failure mode for a cable drive is a broken cable. When this failure occurs depends most strongly on the rated breaking strength of the cable, the actual cable load and the ratio of the cable diameter to the pulley diameter (SAVA[10] recommends a $\frac{d}{D}$ ratio of 16 or more, depending on the cable construction). There is a trade-off between load capacity and fatigue life, i.e for a given drive, increasing the working load decreases the working life of the drive. Break strength and fatigue life data are available from some cable manufacturers (SAVA[10]) but the accuracy of the fatigue data is unclear.

\section{Efficiency}

The theoretical efficiency increases as the radii $R_{i}$ and the cable stiffness $\mathrm{EA}_{\mathrm{i}}$ for each stage increase but decreases as we increase the applied load $\mathrm{M}$ or the number of stages $k$ (see equation (2-118)). However, the theoretical efficiencies for cable drives rarely fall outside the $96-99 \%$ range. Additional losses result from bearing friction which increases as we increase $\mathrm{M}, \mathrm{T}_{0_{\mathrm{i}}}$ or $k$. The magnitude of the bearing losses depends strongly on the cable rigging used in the drive; in crossed drives with short center-to-center distances the net bearing loads due to the pretension $\mathrm{T}_{0}$ are nearly zero whereas the pretension induced bearing loads in an uncrossed drive approach two times the pretension $\mathrm{T}_{0}$. 


\section{Chapter 6: Designing Cable Drives}

\subsubsection{Design Parameters}

\section{Pretension}

We pretension a cable drive to remove backlash from the mechanism. This preload must be large enough to prevent the cables from going slack even under the worst case loading situation. However increasing the pretension decreases load capacity/fatigue life (by increasing cable stress) and increases stiction and friction forces (by increasing bearing loads). Surprisingly, however, increasing pretension also increases the stiffness of the drive and reduces the hysteresis deflection (essentially by increasing the friction forces acting on the cable. These forces reduce the elongation of the wrapped cable in the drive). Thus the pretension gives us a means of changing the physical characteristics (i.e. stiffness and hysteresis deflection) of drive after it has been built without making any modifications to its parts.

\section{Pulley Radii}

Output Pulley: Increasing the size of the output pulley increases the stiffness, fatigue life/ load capacity and reduction ratio of the drive. The only real drawback is that this also increases the size of the drive.

Input Pulley: Increasing the size of the input pulley increases the fatigue life/load capacity of the drive but reduces the reduction ratio. If the reduction ratio is held fixed, increasing the size of the input pulley can dramatically increase the size of the drive because the size of the output pulley must be increased by the same factor.

\section{Number of Stages}

For a given reduction ratio a multistage drive will typically be more compact than a single stage drive but will also have a lower stiffness (because the pulleys are smaller), lower efficiency and higher stiction/friction forces (because of the additional bearings).

\section{Range of Motion}

Range of motion affects only the size of the drive, primarily by requiring that the pulleys be wide enough to hold all of the wrapped cable necessary to enable the desired range of motion.

\section{Pulley Surface Features}

By pulley surface features we mean things like grooves, surface finish, surface material or coating. All of these things can affect the resultant coefficient of friction $\mu$ and therefore can impact the stiffness and hysteresis of the drive. Increasing $\mu$ increases stiffness and decreases the hysteresis deflection. 
Performance Characteristics and Design Parameters: Design

\section{Cable Diameter}

Increasing the cable diameter increases the EA value and therefore increase the drive's stiffness. However the larger cable diameter will likely reduce the fatigue life unless the pulley diameters are increased proportionally (which increases the size of the drive).

\section{Cable Construction}

For steel cables cable construction refers primarily to the number of individual strands wound together to create the cable. For a given diameter a cable made from a large number of small diameter strands will typically have a greater fatigue life than a cable made from a few larger diameter strands. However, the break strength and EA values of the two cables will be roughly the same. Thus a cable with a higher number of strands allows us to use smaller pulleys or, conversely, allows us to obtain a higher reduction ratio for a given size drive. Note however that small diameter strands can be more vulnerable to damage from other forces the cable may see, for example, during assembly.

\section{Cable Coating}

A coated cable typically has a much higher fatigue life than an uncoated cable. However, coated cables will likely have lower coefficients of friction than uncoated cables (we have no data on this yet) thus will likely produce a drive with lower stiffness and greater hysteresis deflections.

\section{Cable Rigging}

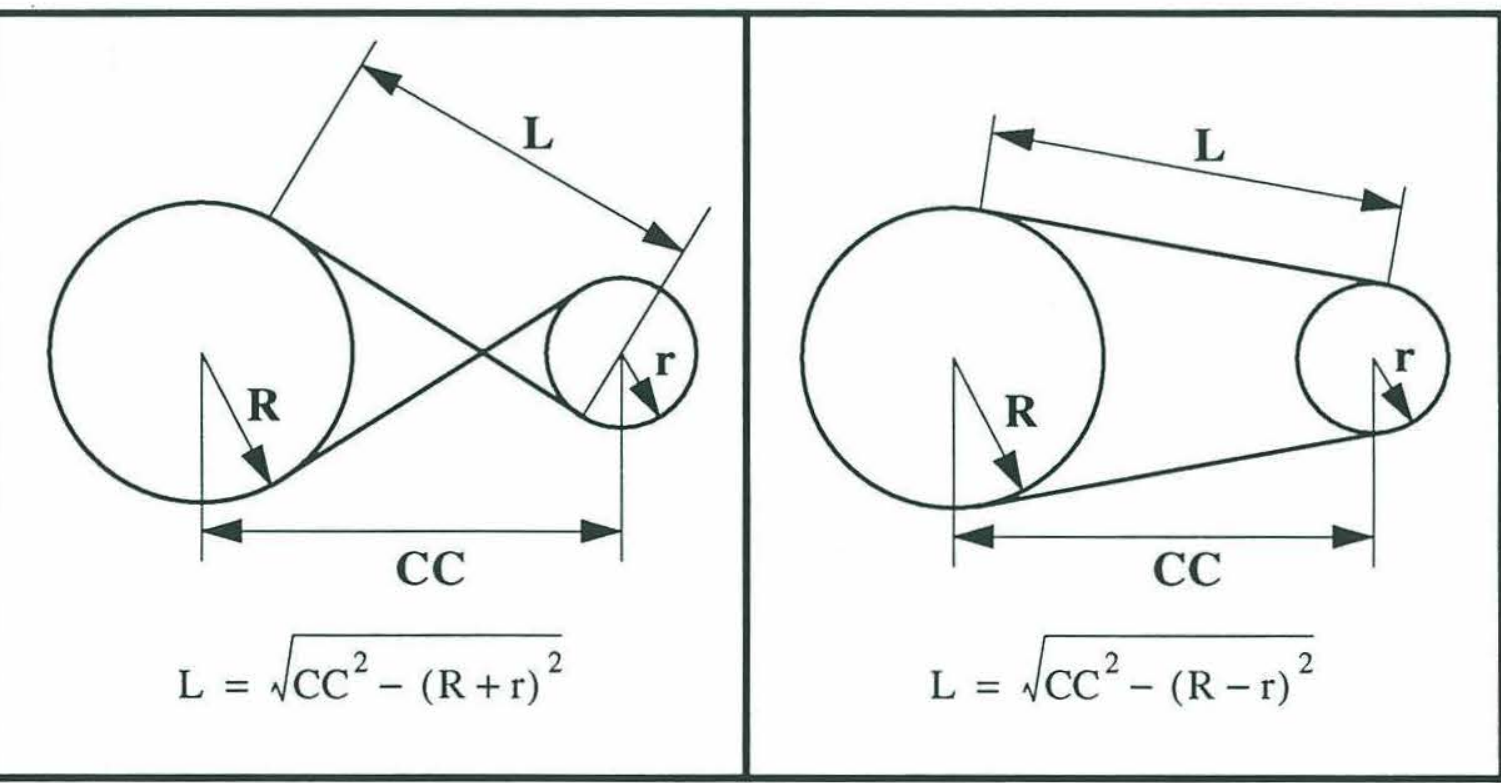

Figure 6.1. Crossed and Uncrossed Methods of Rigging Cable Drives

By cable rigging we mean the manner in which the cables are routed from one pulley to the 


\section{Chapter 6: Designing Cable Drives}

next. As shown in Figure Figure 6.1. there are two types of cable rigging; crossed and uncrossed. Cable rigging impacts stiffness and deflection by its effect on the tangent length $\mathrm{L}$. When the cables are crossed and the pulleys are closed together the tangent length can be made very short, maximizing the stiffness of the drive. The uncrossed drive yields a longer tangent length which results in a lower stiffness for the drive. Cable rigging may have some impact on fatigue life. In the crossed configuration the curvature of the cable is reversed as the drive cycles back and forth which could fatigue the cable more rapidly than in the uncrossed configuration where the curvature is never reversed. We believe that this impact is negligible.

\section{Center-Center Distances}

In both crossed and uncrossed drives increasing the distance between the pulley centers decreases the stiffness and increases the size of the drive.

\subsection{Limitations of the Models/ Future Work}

There are three notable limitations to the cable drive models presented in this report.

\section{The models are static equilibrium models.}

The models assume a Coulomb model for the friction forces between the cable and the pulleys which is true once the system reaches equilibrium. However, our experiments demonstrate the presence of a very significant viscous component to the friction force, indicating that the models can only bound the dynamic behavior of the cable drive. However, the viscous forces will make the actual drive stiffer than predicted, so the equilibrium models give a conservative estimate of the dynamic stiffness of the drive.

\section{Variability of $\mu$}

In our experiments the measured value of $\mu$ for a given cable/pulley combination varied by as much as $\mp 30 \%$ from one trial to the next. Therefore, based on the structure of the deflection and stiffness models, we expect the accuracy of the predictions to be limited to $\mp 30 \%$ as well.

3. Little data available on $\mu$ 's for various cable/pulley material combinations.

To our knowledge the only existing data on the coefficient of friction between cables and pulleys is that presented in this report. More data needs to be generated for to identify $\mu$ when such parameters as cable coating, pulley material and surface properties are varied. 


\section{Appendix A Experimental Apparatus and Procedures}

\section{A.1 Apparatus}

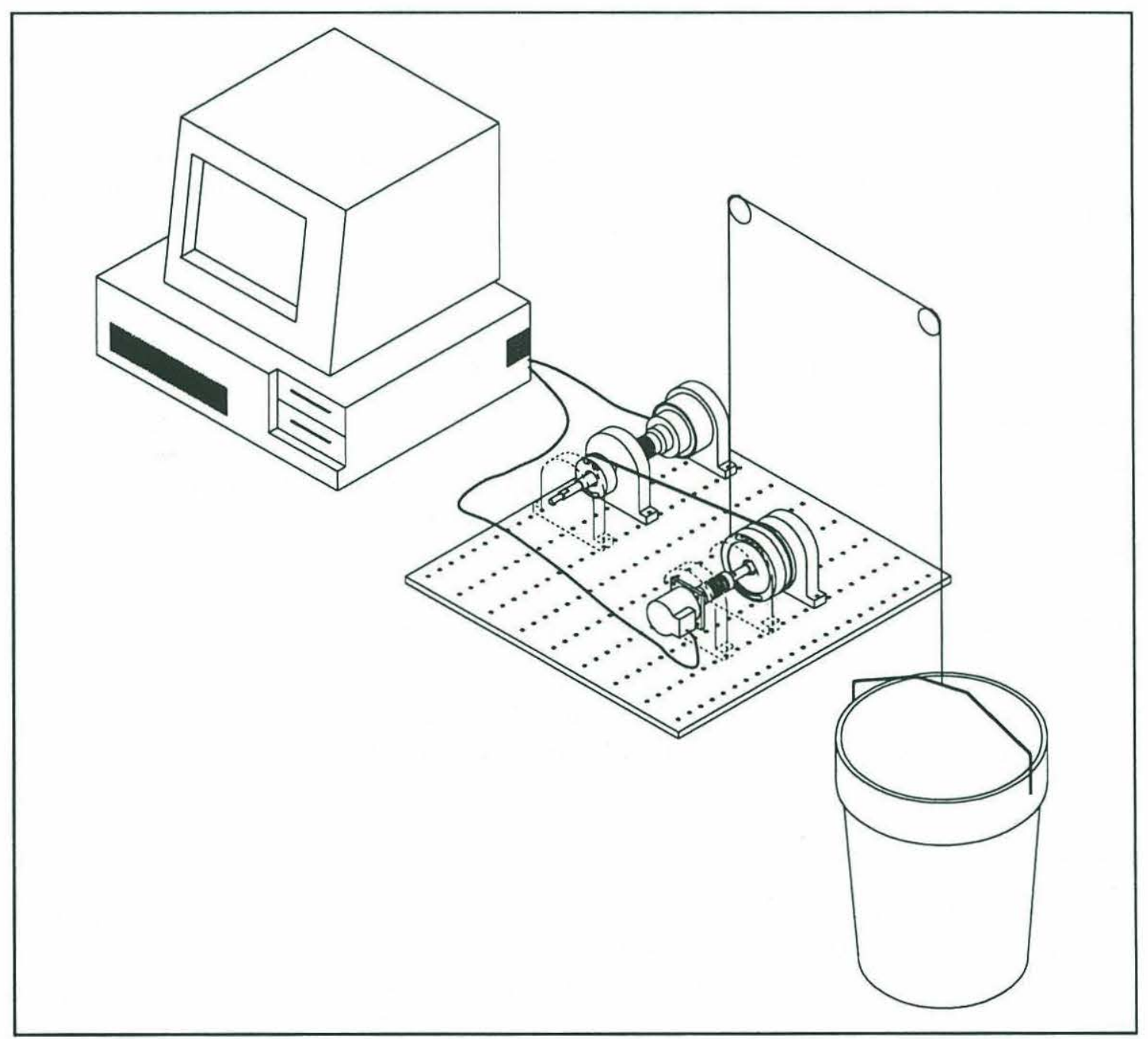

Figure A.1. Experimental Apparatus 


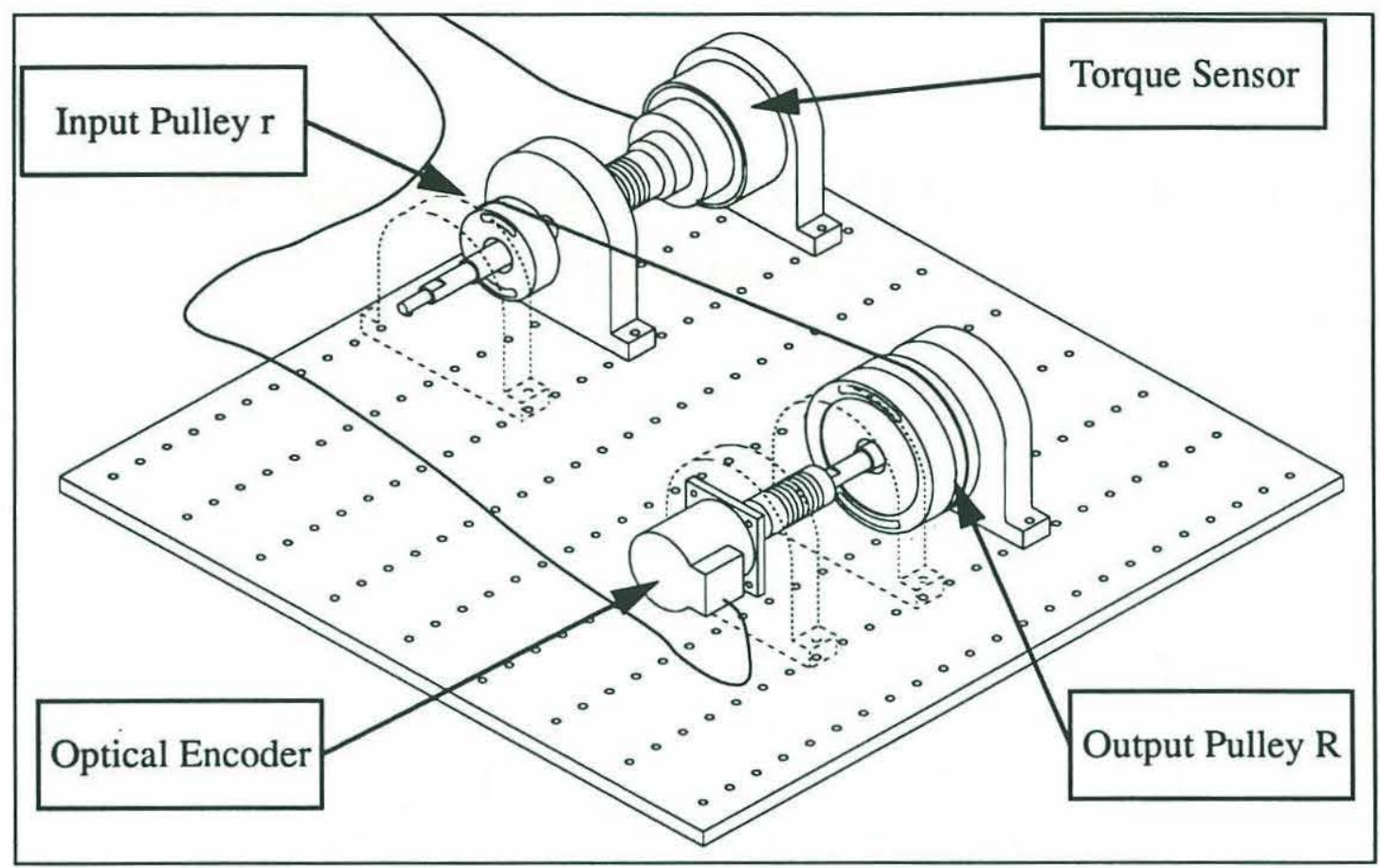

Figure A.2. Detail of Experimental Apparatus

\section{Applying the Load}

To vary the applied load slowly, smoothly and continuously we used a piston type metering pump to pump water into and out of the bucket. The load rate was roughly $.04 \mathrm{lbf}$ of water per second into or out of the bucket.

\section{Base Plate and Bearing/Sensor Mounting Plates}

The plate has an array of precisely positioned threaded holes, each precision counterbored for use with quarter inch shank shoulder bolts. This enabled us to quickly and accurately configure open and closed circuit drives having a variety of pulley diameters and tangent lengths.

\section{Pulleys and Shafts}

We fabricated three different sizes of pulley $(4.875,2.875$ and 1.375 inch O.D.) from two different materials (6061-T6 aluminum and cold rolled steel). The larger pulleys consist of two separate halves; one attaches rigidly to the shaft, the other rotates freely about the shaft on a bearing but can be rigidly clamped to the other half. This design permits us to pretension the closed circuit drives as described below. 
Experimental Procedure

\section{Sensors}

To measure the angular deflection of a drive's rotating pulley we used a high resolution $(100,000$ counts per revolution) optical encoder (Heidenhain Corp., model ROD-5791A09). A standard Helical flex coupling attached the encoder shaft to the shaft of the pulley.

We measured the applied moment by fixing the shaft of the drive's other pulley to the zaxis of a six axis force/torque sensor ( $\mathrm{JR}^{3}$ Company). As configured for our experiments the sensor's resolution was .095 in-lbf. We used a modified Helical coupling to lock the pulley shaft to axis of the torque sensor and found the torsional stiffness of the sensor/ coupling assembly to be 1205 in-lbf per radian.

\section{Cables}

Every drive we configured used 0.018 inch O.D. nylon coated cable, $7 \times 7$ construction (SAVA Industries, part no. 2018-SN). We prestretched each cable prior to running a series of trials to ensure that we removed all constructional stretch. Standard crimped on brass fittings were used to transmit load from the cables to the pulleys. We measured the EA value of this cable, finding it to be $3666 \pm 150 \mathrm{lbf}$ (the EA value represents the product of the modulus of elasticity and the cross sectional area of the cable).

\section{Data Logging}

A PC-AT collected and recorded the data from the encoder and torque sensor at roughly 11 $\mathrm{Hz}$.

\section{A.2 Experimental Procedure}

The experimental procedure for open and closed circuit trials are identical except for the pretensioning procedure.

\section{Preparation}

1. Construct the drive.

2. Prestretch the drive's cable.

Loading the cable to $60 \%$ of its breaking strength, (i.e.

$0.60 \cdot 40 \mathrm{lbf}=24 \mathrm{lbf}$ ) removes the constructional stretch from the cable.

3. Cable the drive. 


\section{Appendix A: Experimental Apparatus and Procedures}

\section{Running a trial}

1. Turn on electronics 20 minutes prior to trial.

The torque sensor electronics should be allowed to reach thermal equilibrium before running a trial

2. Pretension the drive.

(See below)

3. Start the data logging routine

4. Start the pump

5. Stop the pump when the load reaches the desired level.

6. Wait until the deflection reaches its equilibrium value.

7. Reverse the pump.

8. Stop the pump when the load reaches its original value.

9. Wait until deflection reaches equilibrium again

10. Stop data logging routine.

\section{Pretensioning an Open Circuit}

We place a premeasured weight of water into the bucket and then raise and lower the bucket by turning the input pulley through the full range of motion permitted by the cable linking the two pulleys. We then lock the shaft of the input pulley to the torque sensor.

\section{Pretensioning an Closed Circuit}

We place a premeasured weight of water into the bucket and unclamp the two halves of the output pulley so that they can rotate freely with respect to each other. Rotating one half will cause the input pulley to turn, which in turn causes the other half of the output pulley to rotate as well. Attaching the bucket's cable to the latter half of the output pulley we turn the other half through the full range of motion permitted by the drive's cables. Upon completion we clamp the two pulley halves together, locking the cable circuit in a state of tension. We finally empty the bucket and then lock the input pulley to the torque sensor.

\section{A.3 Torque Sensor Compliance}

The measured deflection $\theta_{\mathrm{m}}$ is the sum of the drive deflection and the deflection of the torque sensor/shaft coupling. Thus the drive deflection $\theta$ is

$$
\theta=\theta_{\mathrm{m}}-\frac{\mathrm{r}}{\mathrm{R}} \frac{\stackrel{\circ}{\mathrm{M}}}{\mathrm{k}_{\mathrm{FTS}}}
$$




\section{Torque Sensor Compliance}

where $\mathrm{r}$ is the radius of the pulley fixed to the torque sensor, $\mathrm{R}$ is the radius of the rotating

pulley and $\mathrm{k}_{\mathrm{FTs}}=1205 \frac{\mathrm{in} \cdot \mathrm{lbf}}{\mathrm{rad}}$ is the torsional stiffness of the torque sensor/coupling assembly. 
Appendix A: Experimental Apparatus and Procedures 


\section{Appendix $B$ Coefficients of Friction for Open and Closed Circuit Experiments}

Figures C.1. through C.5. show plots of the true and acting coefficients of frictions (and their estimated uncertainties) for each open circuit trial along while the accompanying tables give the parameter values used in these experiments.

\section{B.1 True Coefficient of Friction, Open Circuit Trials}

As described in Chapter 3 we determine the true coefficient of friction from the equilibrium deflection of the drive. For loading this gives us

$$
\mu_{t_{1}}=\frac{T_{0}}{E A} \frac{\left[m_{p k}-\log \left(m_{p k}-1\right)\right]}{\theta_{p k}-\frac{L_{0}}{E A R} m_{p k}}
$$

where $m_{p k}=\frac{M_{p k}}{\mathrm{RT}_{0}}$ and $\theta_{p k}$ are the dimensionless load and equilibrium deflection at the end of the loading cycle. We can also obtain a value for the true coefficient of friction from the unloading data. In this case we find that

$$
\left.\mu_{t_{u}}=\frac{T_{0}}{E A} \frac{\left[2 \sqrt{\left(1+m_{p k}\right)\left(1+m_{f}\right)}-m_{f}-2-\log \left(1+m_{p k}\right)\right.}{E}\right]
$$

where $\mathrm{m}_{\mathrm{f}}=\frac{\mathrm{M}_{\mathrm{f}}}{\mathrm{RT}_{0}}$ and $\theta_{\mathrm{f}}$ are the final dimensionless load and equilibrium deflection reached at the end of the loading cycle. We plot both $\mu_{t_{1}}$ and $\mu_{t_{u}}$ for each trial in Figures C.1. through C.5. 


\section{B.1 Trials 36 through 85: Single Pulley Tests}
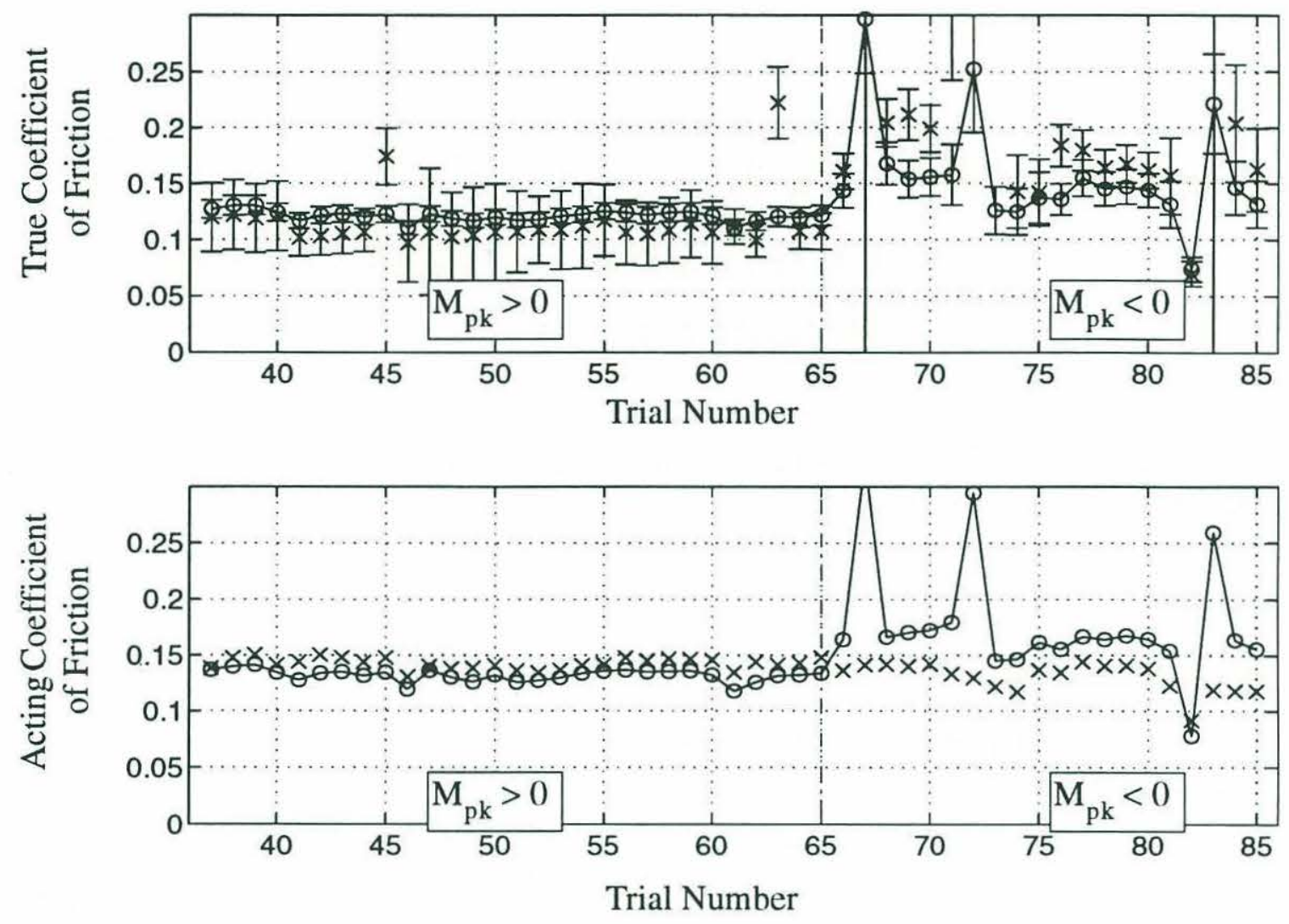

Figure C.1. Loading (o) and Unloading (x) Coefficients of Friction, Trials 36-85

\begin{tabular}{|l|l|l|l|r|c|}
\hline Trial & $\mathrm{R}$ (in) & $\mathrm{r}$ (in) & $\mathrm{L}$ (in) & $\mathrm{T}_{0}(\mathrm{lbf})$ & $\mathrm{M}_{\mathrm{pk}}$ \\
\hline \hline $36-40$ & $2.438 \mathrm{a}$ & 0.000 & 5.50 & 4.33 & + \\
\hline $41-45$ & $2.438 \mathrm{a}$ & 0.000 & 5.50 & 6.54 & + \\
\hline $46-50$ & $2.438 \mathrm{a}$ & 0.000 & 5.50 & 2.13 & + \\
\hline $51-55$ & $2.438 \mathrm{a}$ & 0.000 & 2.45 & 2.13 & + \\
\hline $56-60$ & $2.438 \mathrm{a}$ & 0.000 & 2.45 & 4.33 & + \\
\hline $61-65$ & $2.438 \mathrm{a}$ & 0.000 & 2.45 & 6.54 & + \\
\hline $66-70$ & $2.438 \mathrm{a}$ & 0.000 & 2.45 & 15.36 & - \\
\hline $71-75$ & $2.438 \mathrm{a}$ & 0.000 & 2.45 & 8.74 & - \\
\hline $76-80$ & $2.438 \mathrm{a}$ & 0.000 & 5.50 & 15.36 & - \\
\hline $81-85$ & $2.438 \mathrm{a}$ & 0.000 & 5.50 & 8.74 & - \\
\hline
\end{tabular}

Table C.1. Parameter Values for Open Circuit Trials 36 through 85 


\section{B.1 Trials 86 through 110: Single Pullev Tests}
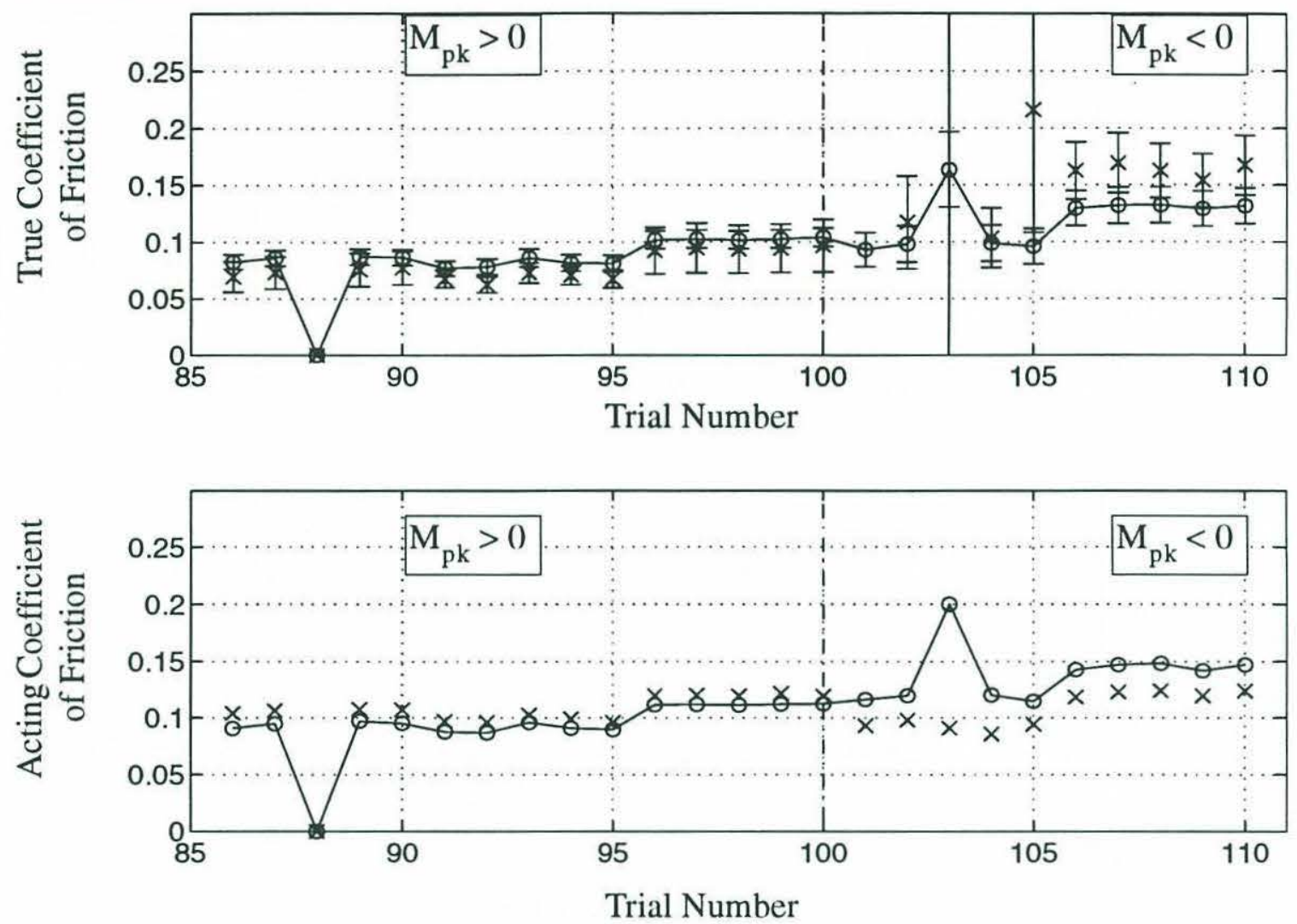

Figure C.2 Loading (o) and Unloading (x) Coefficients of Friction, Trials 86-110

\begin{tabular}{|l|r|r|r|r|c|}
\hline \multicolumn{1}{|c|}{ Trial } & $\mathrm{R}($ in) & $\mathrm{r}$ (in) & $\mathrm{L}$ (in) & $\mathrm{T}_{0}(\mathrm{lbf})$ & $\mathrm{M}_{\mathrm{pk}}$ \\
\hline \hline $86-90$ & $1.438 \mathrm{a}$ & 0.000 & 5.86 & 5.43 & + \\
\hline$(88)$ & void & void & void & void & void \\
\hline $91-95$ & $1.438 \mathrm{a}$ & 0.000 & 5.86 & 8.74 & + \\
\hline $96-100$ & $1.438 \mathrm{a}$ & 0.000 & 5.86 & 2.13 & + \\
\hline $101-105$ & $1.438 \mathrm{a}$ & 0.000 & 5.86 & 8.74 & - \\
\hline $106-110$ & $1.438 \mathrm{a}$ & 0.000 & 5.86 & 15.36 & - \\
\hline
\end{tabular}

Table C.2. Parameter Values for Open Circuit Trials 86 through 110 


\section{B.1 Trials 111 through 135: Single Pullev Tests}
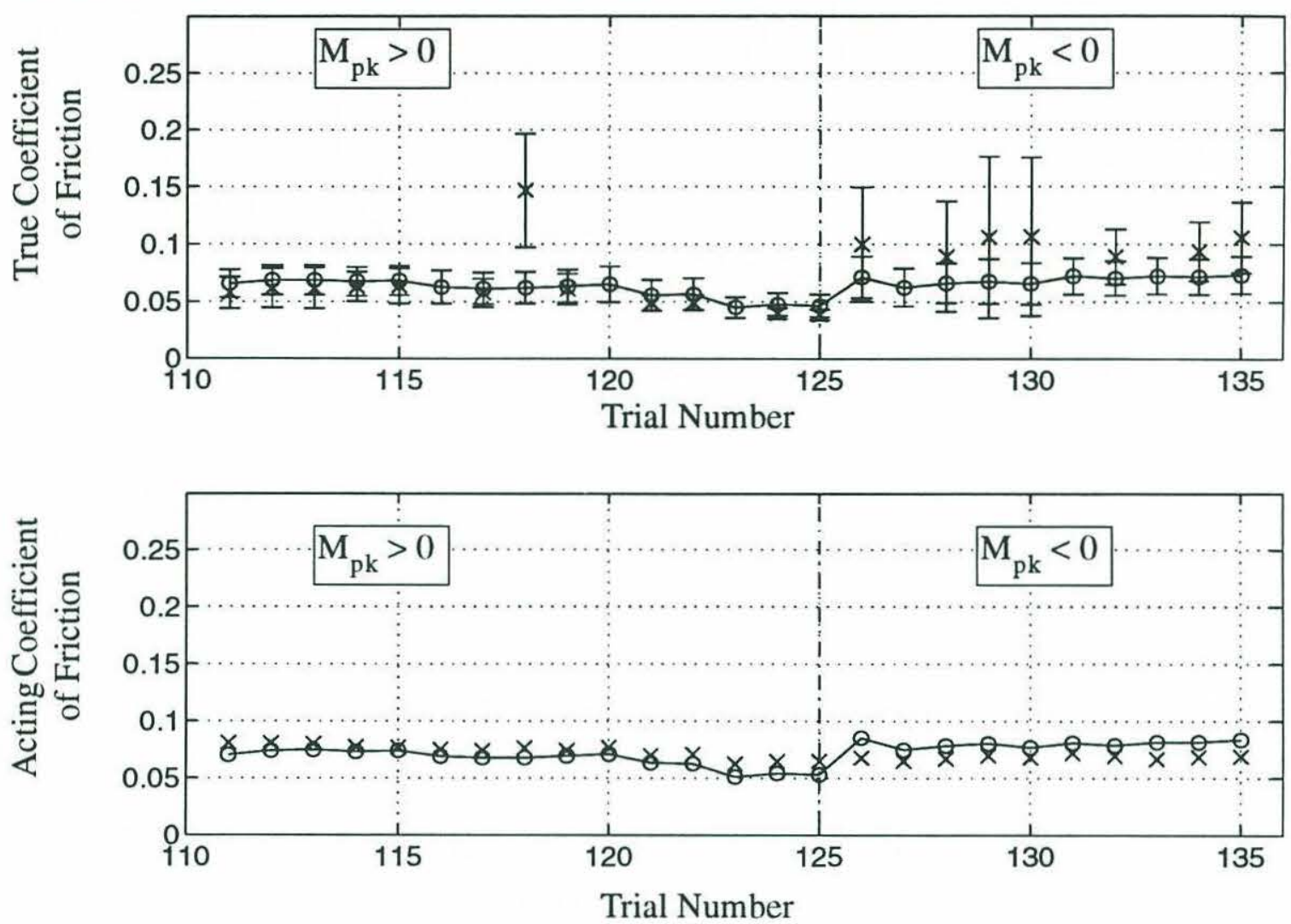

Figure C.3 Loading (o) and Unloading (x) Coefficients of Friction, Trials 111-135

\begin{tabular}{|c|c|c|c|c|c|}
\hline Trial & $\mathrm{R}$ (in) & $\mathrm{r}$ (in) & $\mathrm{L}$ (in) & $\mathrm{T}_{0}(\mathrm{lbf})$ & $\mathrm{M}_{\mathrm{pk}}$ \\
\hline \hline $111-115$ & $0.688 \mathrm{a}$ & 0.000 & 5.20 & 2.13 & + \\
\hline $116-120$ & $0.688 \mathrm{a}$ & 0.000 & 5.20 & 5.43 & + \\
\hline $121-125$ & $0.688 \mathrm{a}$ & 0.000 & 5.20 & 8.74 & + \\
\hline $126-130$ & $0.688 \mathrm{a}$ & 0.000 & 5.20 & 8.74 & - \\
\hline $131-135$ & $0.688 \mathrm{a}$ & 0.000 & 5.20 & 15.36 & - \\
\hline
\end{tabular}

Table C.3. Parameter Values for Open Circuit Trials 111 through 135 


\section{B.1 Trials 136 through 172: Dual Pullev Tests}
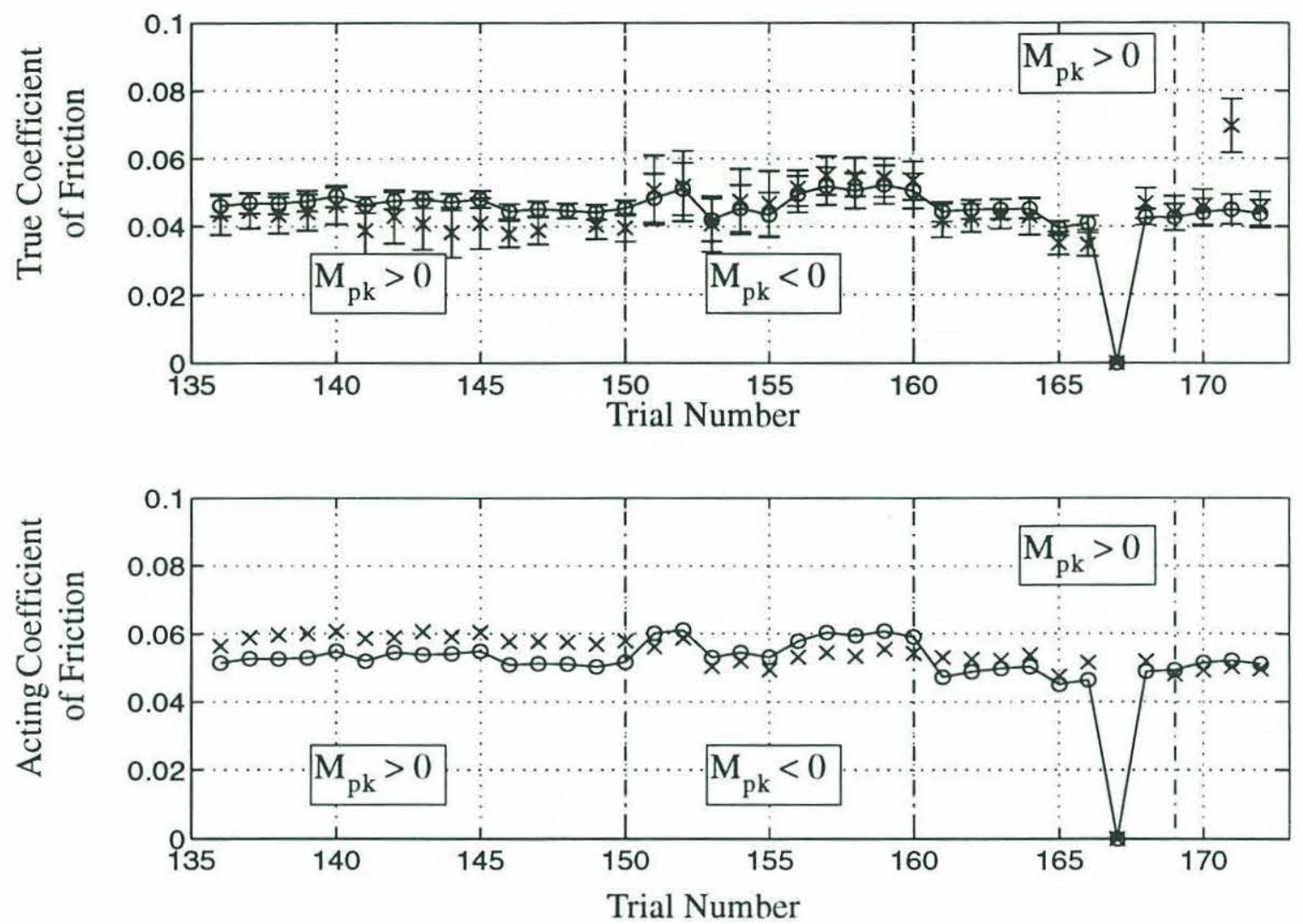

Figure C.4 Loading (o) and Unloading (x) Coefficients of Friction, Trials 136-172

\begin{tabular}{|l|c|c|c|r|c|}
\hline \multicolumn{1}{|c|}{ Trial } & $\mathrm{R}$ (in) & $\mathrm{r}$ (in) & $\mathrm{L}$ (in) & $\mathrm{T}_{0}$ (lbf) & $\mathrm{M}_{\mathrm{pk}}$ \\
\hline \hline $136-140$ & $1.438 \mathrm{a}$ & $2.438 \mathrm{a}$ & 5.41 & 2.13 & + \\
\hline $141-145$ & $1.438 \mathrm{a}$ & $2.438 \mathrm{a}$ & 5.41 & 5.43 & + \\
\hline $146-150$ & $1.438 \mathrm{a}$ & $2.438 \mathrm{a}$ & 5.41 & 8.74 & + \\
\hline $151-155$ & $1.438 \mathrm{a}$ & $2.438 \mathrm{a}$ & 5.41 & 8.74 & - \\
\hline $156-160$ & $1.438 \mathrm{a}$ & $2.438 \mathrm{a}$ & 5.41 & 15.36 & - \\
\hline \hline $161-164$ & $1.438 \mathrm{~s}$ & $2.438 \mathrm{a}$ & 5.41 & 2.13 & + \\
\hline $165-168$ & $1.438 \mathrm{~s}$ & $2.438 \mathrm{a}$ & 5.41 & 8.74 & + \\
\hline$(167)$ & void & void & void & void & void \\
\hline $169-172$ & $1.438 \mathrm{~s}$ & $2.438 \mathrm{a}$ & 5.41 & 15.36 & - \\
\hline
\end{tabular}

Table C.4. Parameter Values for Open Circuit Trials 136 through 172 


\section{B.1 Trials 173 through 188: Dual Pullev Tests}

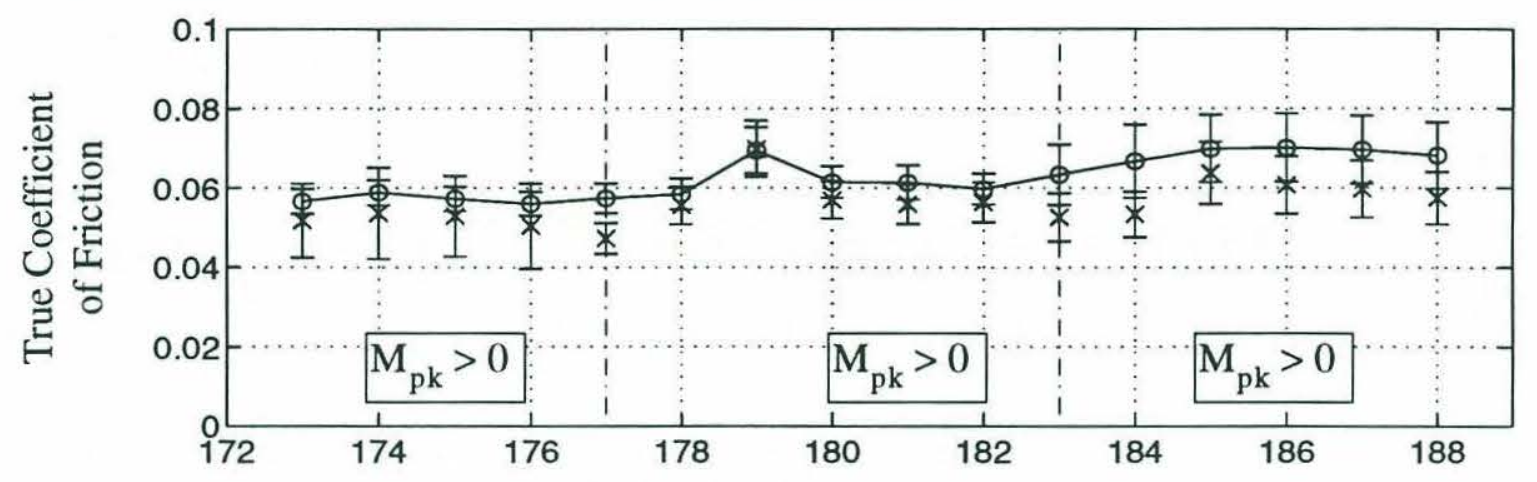

Trial Number

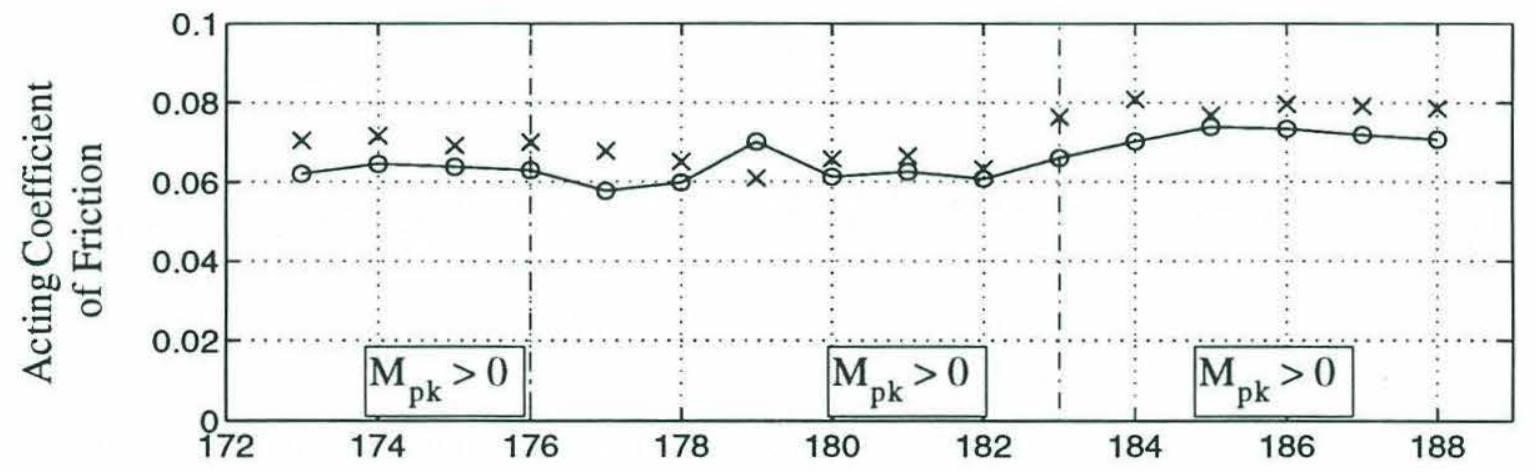

Trial Number

Figure C.5 Loading (o) and Unloading (x) Coefficients of Friction, Trials 173-188

\begin{tabular}{|c|c|c|c|c|c|}
\hline Trial & $\mathrm{R}$ (in) & $\mathrm{r}$ (in) & $\mathrm{L}$ (in) & $\mathrm{T}_{0}$ (lbf) & $\mathrm{M}_{\mathrm{pk}}$ \\
\hline \hline $173-176$ & $1.438 \mathrm{~s}$ & $1.438 \mathrm{a}$ & 5.41 & 2.13 & + \\
\hline \hline $177-182$ & $2.438 \mathrm{~s}$ & $2.438 \mathrm{a}$ & 0.31 & 12.05 & + \\
\hline $183-188$ & $2.438 \mathrm{~s}$ & $2.438 \mathrm{a}$ & 0.31 & 12.05 & + \\
\hline
\end{tabular}

Table C.5. Parameter Values for Open Circuit Trials 173 through 188 
Closed Circuit Experiments: Trials 1 through 30: Dual Pulley Tests

\section{B.1 Closed Circuit Experiments: Trials 1 through 30: Dual Pullev Tests}

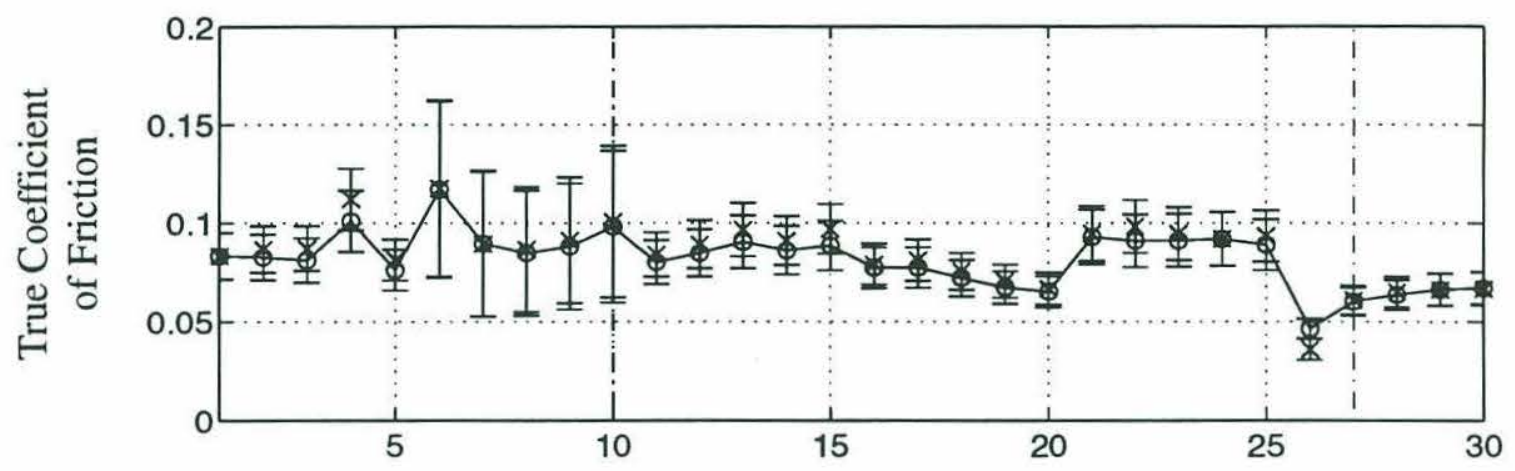

Trial Number

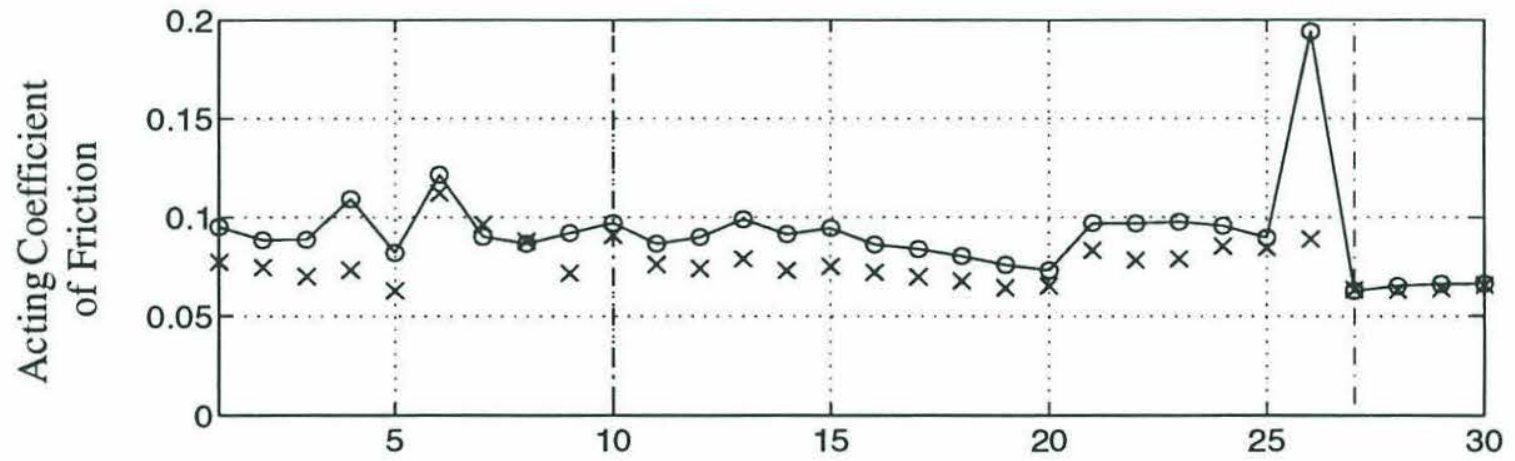

Trial Number

Figure C.6 Loading (o) and Unloading (x) Coefficients of Friction, Trials 1-30

\begin{tabular}{|l|l|c|c|c|c|}
\hline \multicolumn{1}{|c|}{ Trial } & $\mathrm{R}$ (in) & $\mathrm{r}$ (in) & $\mathrm{L}$ (in) & $\mathrm{T}_{0}(\mathrm{lbf})$ & $\mathrm{M}_{\mathrm{pk}}$ \\
\hline \hline $1-5$ & $2.438 \mathrm{~s}$ & $2.438 \mathrm{a}$ & 0.31 & 5.47 & + \\
\hline $6-10$ & $2.438 \mathrm{~s}$ & $2.438 \mathrm{a}$ & 0.31 & 1.33 & + \\
\hline $11-15$ & $2.438 \mathrm{~s}$ & $2.438 \mathrm{a}$ & 0.31 & 7.54 & + \\
\hline $16-20$ & $2.438 \mathrm{~s}$ & $2.438 \mathrm{a}$ & 0.31 & 7.54 & - \\
\hline $21-25$ & $2.438 \mathrm{~s}$ & $2.438 \mathrm{a}$ & 0.31 & 7.54 & - \\
\hline 26 & $2.438 \mathrm{~s}$ & $2.438 \mathrm{a}$ & 0.31 & 7.54 & - \\
\hline $27-30$ & $2.438 \mathrm{~s}$ & $2.438 \mathrm{a}$ & 0.31 & 7.54 & + \\
\hline
\end{tabular}

Table C.6. Parameter Values for Closed Circuit Experiments 


\section{Appendix C Special Case II: Exact Tensions During Unloading}

To find the tensions during unloading for a single stage closed circuit drive with a low GF value we substitute $\mathrm{GF} \approx 0$ into the equilibrium equation (4-22) to get

$$
2\left(\sqrt{\tau_{h_{p k}}\left(\tau_{1}+m\right)}-\sqrt{\tau_{l_{p k}} \tau_{1}}\right)-m+\log \frac{\tau_{l_{p k}}}{\tau_{h_{p k}}}=0
$$

Since GF $\approx 0$ we apply equations (4-42) and (4-44) to find the peak tensions $\tau_{1_{\mathrm{pk}}}$ and $\tau_{\mathrm{h}_{\mathrm{pk}}}$ associated with the peak moment $\mathrm{m}_{\mathrm{pk}}$, finding them to be

$$
\begin{gathered}
\tau_{l_{p k}}=\frac{m_{p k}}{e^{m_{p k}}-1} \\
\tau_{h_{p k}}=\frac{m_{p k} e^{m_{p k}}}{e^{m_{p k}}-1}
\end{gathered}
$$

Thus the last term in equation (D-1) becomes

$$
\log \frac{\tau_{\mathrm{l}_{\mathrm{pk}}}}{\tau_{\mathrm{h}_{\mathrm{pk}}}}=\log \frac{1}{\mathrm{e}^{\mathrm{m}_{\mathrm{pk}}}}=-\mathrm{m}_{\mathrm{pk}}
$$

and we can rewrite equation (D-1) as

$$
2 \sqrt{\tau_{h_{p k}}\left(\tau_{1}+m\right)}=2 \sqrt{\tau_{l_{p k}} \tau_{1}}+\left(m+m_{p k}\right)
$$

Squaring both sides and rearranging terms we get 


$$
\left(\tau_{h_{p k}}-\tau_{l_{p k}}\right) \tau_{1}+\tau_{h_{p k}} m-\frac{\left(m+m_{p k}\right)^{2}}{4}=\left(m+m_{p k}\right) \sqrt{\tau_{l_{p k}} \tau_{1}}
$$

We recognize that $\tau_{\mathrm{h}_{\mathrm{pk}}}-\tau_{\mathrm{l}_{\mathrm{pk}}}=\mathrm{m}_{\mathrm{pk}}$ and then divide through by $\mathrm{m}_{\mathrm{pk}}$ to get

$$
\tau_{1}+\frac{m}{m_{p k}} \tau_{h_{p k}}-\frac{\left(m+m_{p k}\right)^{2}}{4 m_{p k}}=\frac{\left(m+m_{p k}\right)}{m_{p k}} \sqrt{\tau_{1_{p k}} \tau_{1}}
$$

Squaring both sides and skipping over several steps of algebra gives us

$$
\tau_{1}^{2}+2\left(\frac{m}{m_{p k}} \tau_{h_{p k}}-\left(1+2 \frac{\tau_{l_{p k}}}{m_{p k}}\right) \frac{\left(m+m_{p k}\right)^{2}}{4 m_{p k}}\right) \tau_{1}+\left(\frac{m}{m_{p k}} \tau_{h_{p k}}-\frac{\left(m+m_{p k}\right)^{2}}{4 m_{p k}}\right)^{2}=0
$$

This can be solved using the quadratic equation.

$$
\tau_{1}=-\frac{b}{2} \pm \frac{1}{2} \sqrt{b^{2}-4 c}
$$

Where

$$
\mathrm{b}=2\left(\frac{\mathrm{m}}{\mathrm{m}_{\mathrm{pk}}} \tau_{\mathrm{h}_{\mathrm{pk}}}-\left(1+2 \frac{\tau_{\mathrm{l}_{\mathrm{pk}}}}{\mathrm{m}_{\mathrm{pk}}}\right) \frac{\left(\mathrm{m}+\mathrm{m}_{\mathrm{pk}}\right)^{2}}{4 \mathrm{~m}_{\mathrm{pk}}}\right)
$$

and

$$
c=\left(\frac{m}{m_{p k}} \tau_{h_{p k}}-\frac{\left(m+m_{p k}\right)^{2}}{4 m_{p k}}\right)^{2}
$$

Equation (D-9) gives two solutions for $\tau_{1}$ so we must determine which of these is the correct result. We assume that the peak load $\mathrm{m}_{\mathrm{pk}}$ is not large enough to cause either cable to go slack. If the low tension cable is initially under tension, its tension will only increase as the load $m_{p k}$ is removed, in which case the value of $\tau_{1}$ should always be a positive real number. The solutions for $\tau_{1}$ will always be real if

$$
b^{2}-4 c \geq 0
$$


We will find this easier to prove if we first use equations (D-2) and (D-3) to eliminate $\tau_{1_{\mathrm{pk}}}$ and $\tau_{\mathrm{h}_{\mathrm{pk}}}$ from the two coefficients $\mathrm{b}$ and $\mathrm{c}$. After a lot of algebraic manipulation $\mathrm{b}$ and $\mathrm{c}$ become

$$
b=\frac{2}{y}\left(m \frac{e^{m_{p k}}}{e^{m_{p k}}-1}\right)\left[y-\left(1+e^{-m_{p k}}\right)\right]
$$

and

$$
c=\frac{1}{y^{2}}\left(m \frac{e^{m_{p k}}}{e^{m_{p k}}-1}\right)^{2}\left[y-\left(1-e^{-m_{p k}}\right)\right]^{2}
$$

where

$$
y=\frac{4 \frac{\mathrm{m}}{\mathrm{m}_{\mathrm{pk}}}}{\left(\frac{\mathrm{m}}{\mathrm{m}_{\mathrm{pk}}}+1\right)^{2}}
$$

Substituting these forms into $\sqrt{\mathrm{b}^{2}-4 \mathrm{c}}$ and simplifying yields

$$
\frac{4}{y}\left(m \frac{e^{m_{p k}}}{e^{m_{p k}}-1}\right) \sqrt{e^{-m_{p k}}(1-y)}
$$

The domain of $\frac{m}{m_{p k}}$ is $-\infty \leq \frac{m}{m_{p k}} \leq 1$, (i.e. if we are removing the applied load $m$ then $\frac{m}{m_{p k}}$ can never be greater than 1) for which the corresponding range for $y$ is $-\infty \leq y \leq 1$. The term under the radical in equation (D-16) is therefore always positive so the solutions for $\tau_{1}$ given by equation (D-9) are always real.

To determine which solution is positive we look at the sign of the coefficient $b$. We rewrite b slightly by using equation (D-15) to eliminate the first instance of y to get 


$$
\mathrm{b}=\frac{1}{2}\left(\frac{\mathrm{m}}{\mathrm{m}_{\mathrm{pk}}}+1\right)^{2}\left(\frac{\mathrm{m}_{\mathrm{pk}}}{1-\mathrm{e}^{-\mathrm{m}_{\mathrm{pk}}}}\right)\left(\mathrm{y}-\left(1+\mathrm{e}^{-\mathrm{m}_{\mathrm{pk}}}\right)\right)
$$

The term in the first set of brackets is obviously always positive. By considering the two possible cases of $m_{p k}>0$ and $m_{p k}<0$ we see that the term in the second pair of brackets is also always positive (applying L'Hopital's rule we find that this term equals zero when $\mathrm{m}_{\mathrm{pk}}=0$ ). The final term is always negative because $\mathrm{y}$ is always less than one. Therefore the coefficient $b$ is always negative. The solutions for $\tau_{1}$ are then

$$
\tau_{1}=\frac{1}{4}\left(\frac{\mathrm{m}}{\mathrm{m}_{\mathrm{pk}}}+1\right)^{2}\left(\frac{\mathrm{m}_{\mathrm{pk}}}{1-\mathrm{e}^{-\mathrm{m}_{\mathrm{pk}}}}\right)\left(\left(1+\mathrm{e}^{-\mathrm{m}_{\mathrm{pk}}}-\mathrm{y}\right) \pm 2 \sqrt{\mathrm{e}^{-\mathrm{m}_{\mathrm{pk}}}(1-\mathrm{y})}\right)
$$

Both of these solutions are real and non-negative so we it is not clear which one should be used. We shall not present the proof, but only the "+" solution makes physical sense so the solution for $\tau_{1}$ becomes

$$
\tau_{1}=\frac{1}{4}\left(\frac{\mathrm{m}}{\mathrm{m}_{\mathrm{pk}}}+1\right)^{2}\left(\frac{\mathrm{m}_{\mathrm{pk}}}{1-\mathrm{e}^{-\mathrm{m}_{\mathrm{pk}}}}\right)\left(\left(1+\mathrm{e}^{-\mathrm{m}_{\mathrm{pk}}}-\mathrm{y}\right)+2 \sqrt{\mathrm{e}^{-\mathrm{m}_{\mathrm{pk}}}(1-\mathrm{y})}\right)
$$

Having found $\tau_{1}$ we find the corresponding tension $\tau_{\mathrm{h}}$ in the high tension cable by applying the equilibrium constraint (4-17) to get

$$
\tau_{\mathrm{h}}=\tau_{1}+\mathrm{m}
$$




\section{References}

[1] Bejczy, A.K., Salisbury, K.J., "Kinesthetic Coupling Between Operator and Remote Manipulator", Advances in in Computer Technology, ASME, vol.1, August 1980, pp.197-211.

[2] Cotterill, J.H., Applied Mechanics, 3rd ed. rev,; London, England: Macmillan and Co., 1892 , pp. 250-253.

[3] DiPietro, D.M., "Development of an Actively Compliant Underwater Manipulator", Master's thesis, Woods Hole Oceanographic Institution/Massachusetts Institute of Technology, December 1988.

[4] Euler, unknown, 1765.

[5] Goertz, R.C., "Fundamentals of Genral Purpose Manipulators", Nucleonics,vol. 10, ovember 1952.

[6] Green, W.G., Theory of Machines, Blackie \& Son Limited, London, 1955.

[7] McAffee, et.al., "A New Six-Degree-of-Freedom Force-Reflecting Hand Controller for Space Telerobotics", 24th Aerospace Symposium, 1990.

[8] Reynolds, O. , "On the Efficiency of Belts or Straps as Communicators of Work," The Engineer, vol. 38, 1874, p.396.

[9] Salisbury, J.K., "Design and Control of an Articulated Hand", International Symposium on Design and Synthesis, Tokyo, July 1984

[10] SAVA Industries, Catalog No. 14B, Riverdale, N.J., 07457

[11] Schempf, H., "Comparative Design, Modeling, and Control Analysis of Robotic Transmissions", PhD thesis, Woods Hole Oceanographic Institution/Massachusetts Institute of Technology, August 1990.

[12] Swift, H.W., "Power Transmission by Belts: An Investigation of Fundamentals," The Institution of Mechanical Engineers, vol. 1, 1928.

[13] Tordion, G.V., "Creep of an Elastic Belt on a Pulley", ASME Journal of Applied Mechanics, Vol. 26, September 1959, pp. 451-452.

[14] Townsend, W.T., Salisbury, K.J., "The Effect of Coulomb Friction and Stiction on Force Control", IEEE International Conference on Robotics and Automation, Raleigh, NC April 1987

[15] Townsend, W.T., "The Effect of Transmission Design on Force-Controlled Manipulator Performance", PhD thesis, Massachusetts Institute of Technology, April 1988.

[16] Vertut, J., and Coiffet, P., Teleoperations and Robotics: Evolution and Development. In "Robot Technology", pp. 191-194, Prentice Hall, 1986. 
